A-80 deg-MM.pdb

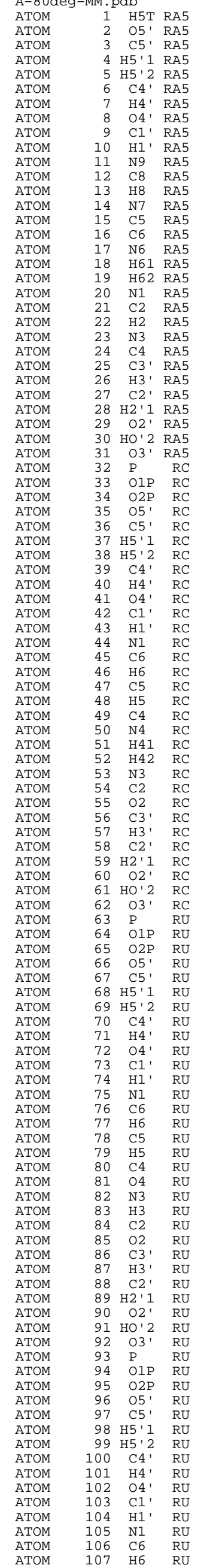

$\begin{array}{llllll}-3.477 & -9.994 & -7.167 & 1.00 & 0.00\end{array}$ $\begin{array}{lllll}-3.767 & -10.204 & -6.251 & 1.00 & 0.00 \\ -3.057 & -11.330 & -5.754 & 1.00 & 0.00\end{array}$ $\begin{array}{lllll}-3.057 & -11.330 & -5.754 & 1.00 & 0.00 \\ -2.787 & -11.995 & -6.575 & 1.00 & 0.00\end{array}$ $\begin{array}{lllll}-3.710 & -11.885 & -5.080 & 1.00 & 0.00\end{array}$ $\begin{array}{lllll}-1.790 & -10.942 & -4.979 & 1.00 & 0.00\end{array}$ $\begin{array}{lllll}-1.346 & -11.857 & -4.582 & 1.00 & 0.00\end{array}$ $\begin{array}{lllll}-0.828 & -10.285 & -5.810 & 1.00 & 0.00\end{array}$ $\begin{array}{lllll}-0.137 & -9.299 & -5.043 & 1.00 & 0.00\end{array}$ $\begin{array}{lllll}0.946 & -9.446 & -5.081 & 1.00 & 0.00\end{array}$ $\begin{array}{lllll}-0.456 & -7.949 & -5.564 & 1.00 & 0.00\end{array}$ $\begin{array}{lllll}-1.498 & -7.578 & -6.375 & 1.00 & 0.00\end{array}$ $\begin{array}{lllll}-1.498 & -7.578 & -6.375 & 1.00 & 0.00 \\ -2.264 & -8.273 & -6.682 & 1.00 & 0.00\end{array}$ $\begin{array}{lllll}-2.264 & -8.273 & -6.682 & 1.00 & 0.00 \\ -1.477 & -6.327 & -6.757 & 1.00 & 0.00\end{array}$ $\begin{array}{lllll}-1.477 & -6.327 & -6.757 & 1.00 & 0.00 \\ -0.322 & -5.830 & -6.117 & 1.00 & 0.00\end{array}$ $\begin{array}{rrrrr}-0.322 & -5.830 & -6.117 & 1.00 & 0.00 \\ 0.338 & -4.576 & -6.031 & 1.00 & 0.00\end{array}$ $\begin{array}{rrrrr}0.338 & -4.576 & -6.031 & 1.00 & 0.00 \\ -0.061 & -3.442 & -6.547 & 1.00 & 0.00\end{array}$ $\begin{array}{rrrrr}-0.061 & -3.442 & -6.547 & 1.00 & 0.00 \\ 0.530 & -2.642 & -6.407 & 1.00 & 0.00\end{array}$ $\begin{array}{rrrrr}0.530 & -2.642 & -6.407 & 1.00 & 0.00 \\ -0.961 & -3.378 & -7.006 & 1.00 & 0.00\end{array}$ $\begin{array}{lllll}1.464 & -4.417 & -5.352 & 1.00 & 0.00\end{array}$ $\begin{array}{lllll}1.981 & -5.482 & -4.772 & 1.00 & 0.00\end{array}$ $\begin{array}{lllll}2.893 & -5.311 & -4.217 & 1.00 & 0.00\end{array}$ $\begin{array}{lllll}1.414 & -6.676 & -4.611 & 1.00 & 0.00\end{array}$ $\begin{array}{lllll}0.300 & -6.811 & -5.389 & 1.00 & 0.00\end{array}$ $\begin{array}{lllll}-2.044 & -9.999 & -3.799 & 1.00 & 0.00 \\ -2.698 & -9.192 & -4.129 & 1.00 & 0.00\end{array}$ $\begin{array}{lllll}-0.620 & -9.467 & -3.604 & 1.00 & 0.00\end{array}$ $\begin{array}{lllll}-0.637 & -8.512 & -3.084 & 1.00 & 0.00\end{array}$ $\begin{array}{lllll}0.255 & -10.401 & -2.972 & 1.00 & 0.00\end{array}$ $\begin{array}{rrrrr}0.255 & -10.401 & -2.972 & 1.00 & 0.00 \\ -0.283 & -11.039 & -2.453 & 1.00 & 0.00\end{array}$ $\begin{array}{lllll}-2.561 & -10.640 & -2.642 & 1.00 & 0.00\end{array}$ $\begin{array}{lllll}-2.561 & -10.640 & -2.642 & 1.00 & 0.00 \\ -3.283 & -9.819 & -1.484 & 1.00 & 0.00\end{array}$ $\begin{array}{lllll}-3.283 & -9.819 & -1.484 & 1.00 & 0.00 \\ -3.761 & -10.775 & -0.462 & 1.00 & 0.00\end{array}$ $\begin{array}{lllll}-4.252 & -8.893 & -2.097 & 1.00 & 0.00\end{array}$ $\begin{array}{lllll}-2.175 & -8.898 & -0.797 & 1.00 & 0.00\end{array}$ $\begin{array}{lllll}-1.108 & -9.418 & -0.023 & 1.00 & 0.00\end{array}$ $\begin{array}{lllll}-0.688 & -10.290 & -0.523 & 1.00 & 0.00\end{array}$ $\begin{array}{lllll}-1.477 & -9.730 & 0.955 & 1.00 & 0.00\end{array}$ $\begin{array}{lllll}0.027 & -8.395 & 0.160 & 1.00 & 0.00\end{array}$ $\begin{array}{lllll}0.870 & -8.925 & 0.599 & 1.00 & 0.00\end{array}$ $\begin{array}{lllll}0.439 & -7.846 & -1.092 & 1.00 & 0.00\end{array}$ $\begin{array}{lllll}0.802 & -6.481 & -0.939 & 1.00 & 0.00\end{array}$ $\begin{array}{lllll}1.824 & -6.341 & -1.302 & 1.00 & 0.00\end{array}$ $\begin{array}{rrrrr}1.824 & -6.341 & -1.302 & 1.00 & 0.00 \\ -0.091 & -5.538 & -1.675 & 1.00 & 0.00\end{array}$ $\begin{array}{lllll}-1.343 & -5.883 & -2.116 & 1.00 & 0.00\end{array}$ $\begin{array}{lllll}-1.343 & -5.883 & -2.116 & 1.00 & 0.00 \\ -1.749 & -6.858 & -1.894 & 1.00 & 0.00\end{array}$ $\begin{array}{lllll}-1.749 & -6.858 & -1.894 & 1.00 & 0.00 \\ -2.072 & -4.990 & -2.825 & 1.00 & 0.00\end{array}$ $\begin{array}{lllll}-3.062 & -5.235 & -3.163 & 1.00 & 0.00\end{array}$ $\begin{array}{lllll}-1.487 & -3.736 & -3.095 & 1.00 & 0.00\end{array}$ $\begin{array}{lllll}-2.139 & -2.845 & -3.755 & 1.00 & 0.00 \\ -1.671 & -1.964 & -3.905 & 1.00 & 0.00\end{array}$ $\begin{array}{lllll}-3.044 & -3.064 & -4.116 & 1.00 & 0.00\end{array}$ $\begin{array}{lllll}-0.333 & -3.351 & -2.623 & 1.00 & 0.00\end{array}$ $\begin{array}{lllll}0.402 & -4.257 & -1.951 & 1.00 & 0.00\end{array}$ $\begin{array}{lllll}1.514 & -3.907 & -1.562 & 1.00 & 0.00\end{array}$ $\begin{array}{lllll}-0.258 & -7.207 & 1.083 & 1.00 & 0.00\end{array}$ $\begin{array}{lllll}-1.272 & -6.840 & 0.911 & 1.00 & 0.00\end{array}$ $\begin{array}{lllll}0.789 & -6.206 & 0.562 & 1.00 & 0.00\end{array}$ $\begin{array}{lllll}0.507 & -5.177 & 0.791 & 1.00 & 0.00\end{array}$ $2.131-6.466$ $\begin{array}{lllll}2.159 & -6.732 & 1.904 & 1.00 & 0.00 \\ -0.021 & -7.550 & 2.439 & 1.00 & 0.00\end{array}$ $\begin{array}{lllll}-0.021 & -7.550 & 2.439 & 1.00 & 0.00 \\ -0.715 & -6.817 & 3.656 & 1.00 & 0.00\end{array}$ $\begin{array}{lllll}-0.715 & -6.817 & 3.656 & 1.00 & 0.00 \\ -0.069 & -7.354 & 4.868 & 1.00 & 0.00\end{array}$ $\begin{array}{lllll}-0.069 & -7.354 & 4.868 & 1.00 & 0.00 \\ -2.173 & -6.987 & 3.508 & 1.00 & 0.00\end{array}$ $\begin{array}{lllll}-0.420 & -5.259 & 3.540 & 1.00 & 0.00\end{array}$ $\begin{array}{lllll}0.837 & -4.678 & 3.870 & 1.00 & 0.00\end{array}$ $\begin{array}{lllll}1.643 & -5.179 & 3.333 & 1.00 & 0.00\end{array}$ $\begin{array}{lllll}1.023 & -4.784 & 4.941 & 1.00 & 0.00 \\ 0.872 & -3.182 & 3.508 & 1.00 & 0.00\end{array}$ $\begin{array}{lllll}0.872 & -3.182 & 3.508 & 1.00 & 0.00\end{array}$ $\begin{array}{lllll}1.796 & -2.750 & 3.887 & 1.00 & 0.00\end{array}$ $\begin{array}{lllll}0.831 & -2.963 & 2.100 & 1.00 & 0.00\end{array}$ $\begin{array}{lllll}0.109 & -1.761 & 1.841 & 1.00 & 0.00\end{array}$ $\begin{array}{lllll}0.751 & -1.060 & 1.299 & 1.00 & 0.00\end{array}$ $\begin{array}{lllll}-1.099 & -2.049 & 1.010 & 1.00 & 0.00\end{array}$ $\begin{array}{lllll}-1.709 & -3.287 & 0.996 & 1.00 & 0.00 \\ -1.327 & -4.078 & 1.624 & 1.00 & 0.00\end{array}$ $\begin{array}{lllll}-1.327 & -4.078 & 1.624 & 1.00 & 0.00 \\ -2.784 & -3.520 & 0.197 & 1.00 & 0.00\end{array}$ $\begin{array}{lllll}-2.784 & -3.520 & 0.197 & 1.00 & 0.00 \\ -3.259 & -4.488 & 0.183 & 1.00 & 0.00\end{array}$ $\begin{array}{rrrrr}-3.259 & -4.488 & 0.183 & 1.00 & 0.00 \\ -3.345 & -2.491 & -0.644 & 1.00 & 0.00\end{array}$ $\begin{array}{lllll}-3.345 & -2.491 & -0.644 & 1.00 & 0.00 \\ -4.333 & -2.620 & -1.360 & 1.00 & 0.00\end{array}$ $\begin{array}{lllll}-4.333 & -2.620 & -1.360 & 1.00 & 0.00 \\ -2.656 & -1.311 & -0.615 & 1.00 & 0.00\end{array}$ $\begin{array}{lllll}-2.987 & -0.558 & -1.194 & 1.00 & 0.00\end{array}$ $\begin{array}{lllll}-1.592 & -1.022 & 0.202 & 1.00 & 0.00\end{array}$ $\begin{array}{lllll}-1.131 & 0.115 & 0.195 & 1.00 & 0.00\end{array}$ $\begin{array}{lllll}-0.279 & -2.375 & 4.108 & 1.00 & 0.00\end{array}$ $\begin{array}{lllll}-1.210 & -2.911 & 3.926 & 1.00 & 0.00\end{array}$ $\begin{array}{lllll}-0.255 & -1.139 & 3.200 & 1.00 & 0.00\end{array}$ $\begin{array}{rrrrr}-1.237 & -0.666 & 3.178 & 1.00 & 0.00 \\ 0.750 & -0.189 & 3.544 & 1.00 & 0.00\end{array}$ $\begin{array}{rrrrr}0.750 & -0.189 & 3.544 & 1.00 & 0.00 \\ 0.553 & 0.654 & 3.070 & 1.00 & 0.00\end{array}$ $\begin{array}{rrrrr}0.553 & 0.654 & 3.070 & 1.00 & 0.00 \\ -0.102 & -2.147 & 5.501 & 1.00 & 0.00\end{array}$ $\begin{array}{lllll}-0.102 & -2.147 & 5.501 & 1.00 & 0.00 \\ -1.247 & -1.524 & 6.401 & 1.00 & 0.00\end{array}$ $\begin{array}{lllll}-1.247 & -1.524 & 6.401 & 1.00 & 0.00 \\ -0.923 & -1.894 & 7.793 & 1.00 & 0.00\end{array}$ $\begin{array}{lllll}-2.548 & -1.970 & 5.860 & 1.00 & 0.00\end{array}$ $\begin{array}{lllll}-1.117 & 0.063 & 6.262 & 1.00 & 0.00\end{array}$ $\begin{array}{lllll}-1.757 & 0.951 & 7.178 & 1.00 & 0.00 \\ -1.910 & 0.461 & 8.140 & 1.00 & 0.00\end{array}$ $\begin{array}{lllll}-1.910 & 0.461 & 8.140 & 1.00 & 0.00 \\ -2.735 & 1.245 & 6.797 & 1.00 & 0.00\end{array}$ $\begin{array}{lllll}-0.920 & 2.211 & 7.433 & 1.00 & 0.00\end{array}$ $\begin{array}{lllll}-1.417 & 2.797 & 8.206 & 1.00 & 0.00\end{array}$ $\begin{array}{lllll}0.349 & 1.802 & 7.930 & 1.00 & 0.00\end{array}$ $\begin{array}{lllll}1.360 & 2.520 & 7.261 & 1.00 & 0.00\end{array}$ $\begin{array}{lllll}1.580 & 3.413 & 7.848 & 1.00 & 0.00\end{array}$ $\begin{array}{lllll}2.586 & 1.702 & 7.087 & 1.00 & 0.00 \\ 2.538 & 0.462 & 6.489 & 1.00 & 0.00\end{array}$ $\begin{array}{lllll}2.538 & 0.462 & 6.489 & 1.00 & 0.00 \\ 1.581 & 0.050 & 6.178 & 1.00 & 0.00\end{array}$ 


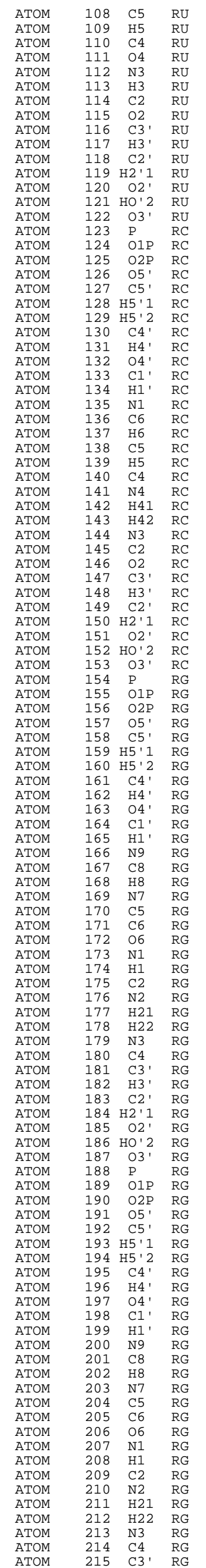

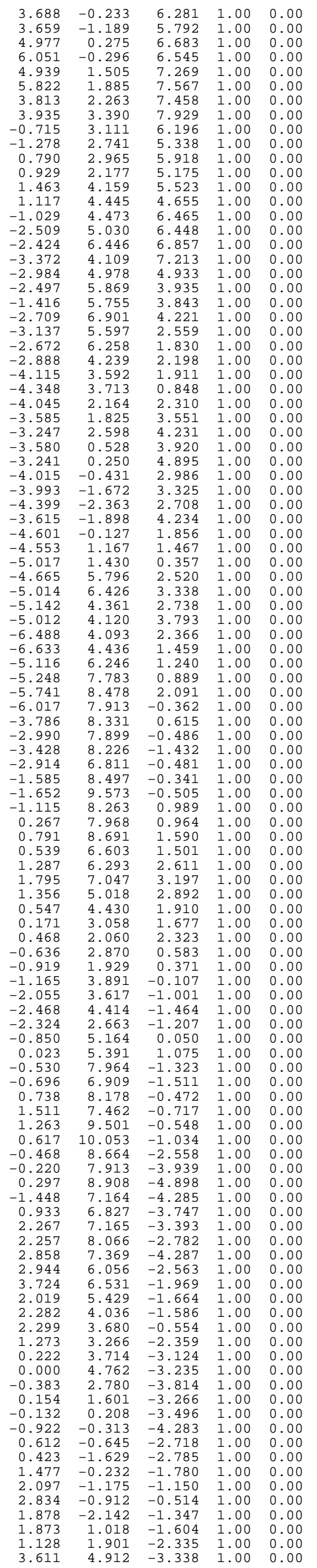




\begin{tabular}{|c|c|c|c|}
\hline ATOM & 216 & $\mathrm{H}^{\prime}{ }^{\prime}$ & RG \\
\hline ATOM & 217 & C2' & RG \\
\hline ATOM & 218 & $\mathrm{H} 2{ }^{\prime} 1$ & RG \\
\hline ATOM & 219 & $02^{\prime}$ & RG \\
\hline ATOM & 220 & HO' 2 & RG \\
\hline ATOM & 221 & $03^{\prime}$ & RG \\
\hline ATOM & 222 & P & RU3 \\
\hline ATOM & 223 & O1P & RU3 \\
\hline ATOM & 224 & $\mathrm{O} 2 \mathrm{P}$ & RU3 \\
\hline ATOM & 225 & $05^{\prime}$ & RU3 \\
\hline ATOM & 226 & C5 ' & RU3 \\
\hline ATOM & 227 & H5' 1 & RU3 \\
\hline ATOM & 228 & H5' 2 & RU3 \\
\hline ATOM & 229 & C4 & RU3 \\
\hline ATOM & 230 & H4 ' & RU3 \\
\hline ATOM & 231 & $04{ }^{\prime}$ & RU3 \\
\hline ATOM & 232 & $\mathrm{C} 1^{\prime}$ & RU3 \\
\hline ATOM & 233 & H1 ' & RU3 \\
\hline ATOM & 234 & N1 & RU3 \\
\hline ATOM & 235 & C6 & RU3 \\
\hline ATOM & 236 & H6 & RU3 \\
\hline ATOM & 237 & C5 & RU3 \\
\hline ATOM & 238 & H5 & RU3 \\
\hline ATOM & 239 & C4 & RU3 \\
\hline ATOM & 240 & 04 & RU3 \\
\hline ATOM & 241 & N3 & RU3 \\
\hline ATOM & 242 & H3 & RU3 \\
\hline ATOM & 243 & $\mathrm{C} 2$ & RU3 \\
\hline ATOM & 244 & 02 & RU3 \\
\hline ATOM & 245 & C3' & RU3 \\
\hline ATOM & 246 & H3' & RU3 \\
\hline ATOM & 247 & C2' & RU3 \\
\hline ATOM & 248 & $\mathrm{H} 2{ }^{\prime} 1$ & RU3 \\
\hline ATOM & 249 & $\mathrm{O} 2^{\prime}$ & RU3 \\
\hline ATOM & 250 & HO' 2 & RU3 \\
\hline ATOM & 251 & $03^{\prime}$ & RU3 \\
\hline ATOM & 252 & H3T & RU3 \\
\hline & & & \\
\hline
\end{tabular}

$\begin{array}{rrrrr}2.945 & 4.585 & -4.138 & 1.00 & 0.00 \\ 3.664 & 3.871 & -2.206 & 1.00 & 0.00 \\ 3.807 & 2.863 & -2.587 & 1.00 & 0.00 \\ 4.624 & 4.169 & -1.192 & 1.00 & 0.00 \\ 5.284 & 4.773 & -1.567 & 1.00 & 0.00 \\ 4.909 & 5.227 & -3.831 & 1.00 & 0.00 \\ 5.633 & 4.331 & -4.937 & 1.00 & 0.00 \\ 6.901 & 5.015 & -5.265 & 1.00 & 0.00 \\ 4.661 & 4.059 & -6.010 & 1.00 & 0.00 \\ 5.947 & 2.940 & -4.247 & 1.00 & 0.00 \\ 6.895 & 2.766 & -3.213 & 1.00 & 0.00 \\ 6.688 & 3.439 & -2.383 & 1.00 & 0.00 \\ 7.891 & 2.985 & -3.599 & 1.00 & 0.00 \\ 6.866 & 1.320 & -2.697 & 1.00 & 0.00 \\ 7.521 & 1.253 & -1.830 & 1.00 & 0.00 \\ 5.562 & 0.870 & -2.311 & 1.00 & 0.00 \\ 5.343 & -0.464 & -2.789 & 1.00 & 0.00 \\ 5.036 & -1.111 & -1.964 & 1.00 & 0.00 \\ 4.291 & -0.546 & -3.849 & 1.00 & 0.00 \\ 3.730 & 0.574 & -4.424 & 1.00 & 0.00 \\ 4.066 & 1.549 & -4.125 & 1.00 & 0.00 \\ 2.774 & 0.467 & -5.382 & 1.00 & 0.00 \\ 2.367 & 1.354 & -5.840 & 1.00 & 0.00 \\ 2.323 & -0.818 & -5.849 & 1.00 & 0.00 \\ 1.492 & -0.986 & -6.737 & 1.00 & 0.00 \\ 2.873 & -1.886 & -5.194 & 1.00 & 0.00 \\ 2.553 & -2.806 & -5.452 & 1.00 & 0.00 \\ 3.815 & -1.817 & -4.195 & 1.00 & 0.00 \\ 4.182 & -2.853 & -3.642 & 1.00 & 0.00 \\ 7.392 & 0.333 & -3.734 & 1.00 & 0.00 \\ 7.048 & 0.616 & -4.730 & 1.00 & 0.00 \\ 6.698 & -0.967 & -3.306 & 1.00 & 0.00 \\ 6.586 & -1.636 & -4.163 & 1.00 & 0.00 \\ 7.326 & -1.640 & -2.216 & 1.00 & 0.00 \\ 8.146 & -1.154 & -1.973 & 1.00 & 0.00 \\ 8.811 & 0.250 & -3.694 & 1.00 & 0.00 \\ 9.068 & -0.466 & -4.334 & 1.00 & 0.00 \\ & & & & \end{array}$

A-180 deg-MM.pdb

ATOM

$\begin{array}{lllll}-3.445 & -9.998 & -7.154 & 1.00 & 0.00\end{array}$

$\begin{array}{lllll}-3.445 & -9.998 & -7.154 & 1.00 & 0.00\end{array}$ $\begin{array}{lllll}-3.015 & -11.330 & -5.740 & 1.00 & 0.00\end{array}$ $-2.739-11.994-6.560 \quad 1.00 \quad 0.00$

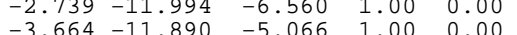
$-3.664-11.890-5.066-1.00-0.00$ $\begin{array}{lllll}-1.752 & -10.931 & -4.965 & 1.00 & 0.00 \\ -1.301 & -11.841 & -4.566 & 1.00 & 0.00\end{array}$ $\begin{array}{lllll}-1.301 & -11.841 & -4.566 & 1.00 & 0.00 \\ -0.793 & -10.268 & -5.796 & 1.00 & 0.00\end{array}$ $\begin{array}{rrrrr}-0.793 & -10.268 & -5.796 & 1.00 & 0.00 \\ -0.110 & -9.277 & -5.028 & 1.00 & 0.00\end{array}$ $\begin{array}{lllll}0.974 & -9.418 & -5.063 & 1.00 & 0.00\end{array}$ $\begin{array}{lllll}-0.435 & -7.929 & -5.552 & 1.00 & 0.00\end{array}$ $\begin{array}{lllll}-1.478 & -7.565 & -6.366 & 1.00 & 0.00\end{array}$ $\begin{array}{lllll}-2.240 & -8.265 & -6.672 & 1.00 & 0.00\end{array}$ $\begin{array}{lllll}-1.462 & -6.315 & -6.751 & 1.00 & 0.00 \\ -0.310 & -5.811 & -6.112 & 1.00 & 0.00\end{array}$ $\begin{array}{lllll}0.346 & -4.555 & -6.030 & 1.00 & 0.00\end{array}$ $\begin{array}{rrrrr}0.0 .055 & -3.424 & -6.552 & 1.00 & 0.00\end{array}$ $\begin{array}{llllll}0.534 & -2.621 & -6.410 & 1.00 & 0.00\end{array}$ $\begin{array}{lllll}-0.955 & -3.363 & -7.011 & 1.00 & 0.00\end{array}$ $\begin{array}{lllll}1.970 & -3.363 & -7.011 & 1.00 & 0.00\end{array}$ $\begin{array}{lllll}1.470 & -4.389 & -5.351 & 1.00 & 0.00 \\ 1.989 & -5.449 & -4.763 & 1.00 & 0.00\end{array}$ $\begin{array}{llll}1.989 & -5.449-4.763 & 1.00 & 0.00\end{array}$ $\begin{array}{lllll}2.899 & -5.273 & -4.207 & 1.00 & 0.00\end{array}$ $\begin{array}{lllll}1.426 & -6.645 & -4.600 & 1.00 & 0.00 \\ 0.314 & -6.787 & -5.379 & 1.00 & 0.00\end{array}$ $\begin{array}{rrrrr}0.314 & -6.787 & -5.379 & 1.00 & 0.00 \\ -2.015 & -9.988 & -3.788 & 1.00 & 0.00\end{array}$ $\begin{array}{lllll}-2.015 & -9.988 & -3.788 & 1.00 & 0.00 \\ -2.674 & -9.186 & -4.119 & 1.00 & 0.00\end{array}$ $\begin{array}{lllll}-0.596 & -9.445 & -3.590 & 1.00 & 0.00\end{array}$ $\begin{array}{lllll}-0.622 & -8.490 & -3.072 & 1.00 & 0.00\end{array}$ $\begin{array}{lllll}0.284 & -10.373 & -2.954 & 1.00 & 0.00\end{array}$ $\begin{array}{lllll}-0.252 & -11.014 & -2.437 & 1.00 & 0.00\end{array}$ $\begin{array}{lllll}-2.531 & -10.633 & -2.632 & 1.00 & 0.00\end{array}$ $\begin{array}{lllll}-3.734 & -10.776 & -0.455 & 1.00 & 0.00\end{array}$ $\begin{array}{lllll}-4.237 & -8.898 & -2.091 & 1.00 & 0.00\end{array}$ $\begin{array}{lllll}-2.162 & -8.888 & -0.789 & 1.00 & 0.00\end{array}$ $\begin{array}{lllll}-2.162 & -8.888 & -0.789 & 1.00 & 0.00 \\ -1.089 & -9.401 & -0.017 & 1.00 & 0.00\end{array}$ $\begin{array}{lllll}-0.660 & -10.266 & -0.520 & 1.00 & 0.00\end{array}$ $\begin{array}{rrrrr}-0.660 & -10.266 & -0.520 & 1.00 & 0.00 \\ -1.454 & -9.719 & 0.960 & 1.00 & 0.00\end{array}$ $\begin{array}{rrrrr}-1.454 & -9.719 & 0.960 & 1.00 & 0.00 \\ 0.033 & -8.364 & 0.167 & 1.00 & 0.00\end{array}$ $\begin{array}{rrrrr}0.033 & -8.364 & 0.167 & 1.00 & 0.00 \\ 0.883 & -8.883 & 0.605 & 1.00 & 0.00\end{array}$ $\begin{array}{rrrrr}0.883 & -8.883 & 0.605 & 1.00 & 0.00 \\ 0.437 & -7.804 & -1.081 & 1.00 & 0.00\end{array}$ $\begin{array}{lllll}0.786 & -6.437 & -0.921 & 1.00 & 0.00\end{array}$ $\begin{array}{lllll}1.810 & -6.289 & -1.275 & 1.00 & 0.00\end{array}$ $\begin{array}{lllll}-0.106 & -5.502 & -1.666 & 1.00 & 0.00\end{array}$ $\begin{array}{lllll}-1.361 & -5.849 & -2.096 & 1.00 & 0.00 \\ -1.770 & -6.820 & -1.856 & 1.00 & 0.00\end{array}$ $\begin{array}{lllll}-2.091 & -4.963 & -2.814 & 1.00 & 0.00\end{array}$ $\begin{array}{lllll}-3.083 & -5.209 & -3.144 & 1.00 & 0.00\end{array}$ $\begin{array}{lllll}-1.501 & -3.716 & -3.105 & 1.00 & 0.00\end{array}$ $\begin{array}{lllll}-2.150 & -2.829 & -3.773 & 1.00 & 0.00 \\ -1.678 & -1.952 & -3.926 & 1.00 & 0.00\end{array}$ $\begin{array}{lllll}-1.678 & -1.952 & -3.926 & 1.00 & 0.00\end{array}$ $\begin{array}{lllll}-3.059 & -3.045 & -4.127 & 1.00 & 0.00 \\ -0.345 & -3.328 & -2.643 & 1.00 & 0.00\end{array}$ $\begin{array}{rrrrr}-0.345 & -3.328 & -2.643 & 1.00 & 0.00 \\ 0.390 & -4.227 & -1.961 & 1.00 & 0.00\end{array}$ $\begin{array}{lllll}1.505 & -3.876 & -1.582 & 1.00 & 0.00\end{array}$ $\begin{array}{lllll}-0.272 & -7.186 & 1.095 & 1.00 & 0.00\end{array}$ $\begin{array}{lllll}-1.291 & -6.836 & 0.924 & 1.00 & 0.00\end{array}$ $\begin{array}{lllll}0.758 & -6.163 & 0.580 & 1.00 & 0.00\end{array}$ $\begin{array}{lllll}0.455 & -5.139 & 0.809 & 1.00 & 0.00\end{array}$ $\begin{array}{lllll}2.101 & -6.400 & 0.985 & 1.00 & 0.00\end{array}$ $\begin{array}{lllll}2.129 & -6.677 & 1.924 & 1.00 & 0.00\end{array}$ $\begin{array}{lllll}-0.031 & -7.538 & 2.448 & 1.00 & 0.00\end{array}$ $\begin{array}{lllll}-0.725 & -6.814 & 3.668 & 1.00 & 0.00\end{array}$ $\begin{array}{lllll}-2.061 & -7.335 & 4.878 & 1.00 & 0.00\end{array}$ $\begin{array}{lllll}-2.181 & -7.005 & 3.535 & 1.00 & 0.00 \\ -0.459 & -5.254 & 3.542 & 1.00 & 0.00\end{array}$ $\begin{array}{rrrrr}-0.459 & -5.254 & 3.542 & 1.00 & 0.00 \\ 0.778 & -4.637 & 3.886 & 1.00 & 0.00\end{array}$ $\begin{array}{lllll}1.600 & -5.080 & 3.323 & 1.00 & 0.00\end{array}$ 


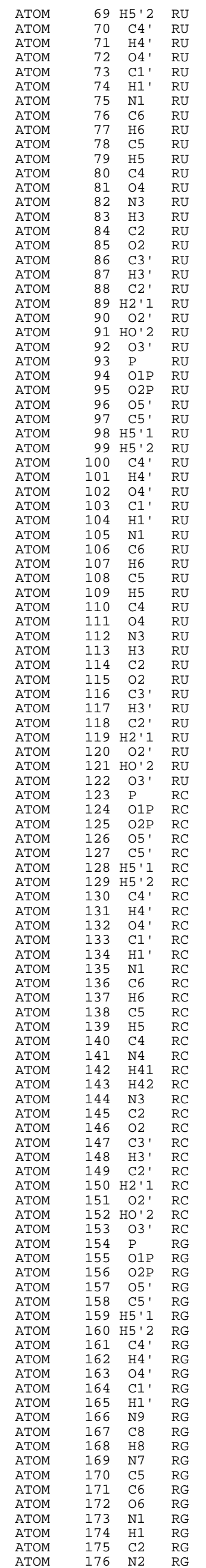

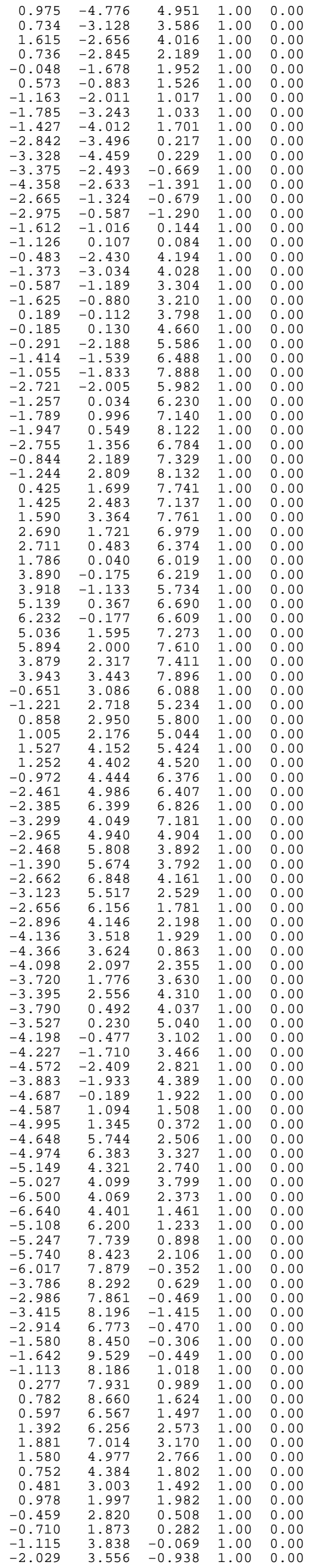




\begin{tabular}{|c|c|c|c|}
\hline ATOM & 177 & H 21 & RG \\
\hline ATOM & 178 & $\mathrm{H} 22$ & RG \\
\hline ATOM & 179 & N3 & RG \\
\hline ATOM & 180 & C4 & RG \\
\hline ATOM & 181 & C3' & RG \\
\hline ATOM & 182 & H3' & RG \\
\hline ATOM & 183 & C2' & RG \\
\hline ATOM & 184 & H2' 1 & RG \\
\hline ATOM & 185 & $02^{\prime}$ & RG \\
\hline ATOM & 186 & $\mathrm{HO}^{\prime} 2$ & RG \\
\hline ATOM & 187 & $03^{\prime}$ & RG \\
\hline ATOM & 188 & P & RG \\
\hline ATOM & 189 & O1P & RG \\
\hline ATOM & 190 & $\mathrm{O} 2 \mathrm{P}$ & RG \\
\hline ATOM & 191 & $05^{\prime}$ & RG \\
\hline ATOM & 192 & C5' & RG \\
\hline ATOM & 193 & H5' 1 & RG \\
\hline ATOM & 194 & H5' 2 & RG \\
\hline ATOM & 195 & C4 & RG \\
\hline ATOM & 196 & H4 ${ }^{\prime}$ & RG \\
\hline ATOM & 197 & $04^{\prime}$ & RG \\
\hline ATOM & 198 & $\mathrm{C} 1^{\prime}$ & RG \\
\hline ATOM & 199 & H1 ' & RG \\
\hline ATOM & 200 & N9 & RG \\
\hline ATOM & 201 & C8 & RG \\
\hline ATOM & 202 & $\mathrm{H} 8$ & RG \\
\hline ATOM & 203 & N7 & RG \\
\hline ATOM & 204 & C5 & RG \\
\hline ATOM & 205 & C6 & RG \\
\hline ATOM & 206 & 06 & RG \\
\hline ATOM & 207 & N1 & RG \\
\hline ATOM & 208 & H1 & RG \\
\hline ATOM & 209 & C2 & RG \\
\hline ATOM & 210 & N2 & RG \\
\hline ATOM & 211 & H21 & RG \\
\hline ATOM & 212 & H 22 & RG \\
\hline ATOM & 213 & N3 & RG \\
\hline ATOM & 214 & C4 & RG \\
\hline ATOM & 215 & C3' & RG \\
\hline ATOM & 216 & H3' & RG \\
\hline ATOM & 217 & C2 ${ }^{\prime}$ & RG \\
\hline ATOM & 218 & H2 ' 1 & RG \\
\hline ATOM & 219 & O2' & RG \\
\hline ATOM & 220 & HO' 2 & RG \\
\hline ATOM & 221 & $03^{\prime}$ & RG \\
\hline ATOM & 222 & $\mathrm{P}$ & RU3 \\
\hline ATOM & 223 & $\mathrm{O} 1 \mathrm{P}$ & RU3 \\
\hline ATOM & 224 & $\mathrm{O} 2 \mathrm{P}$ & RU3 \\
\hline ATOM & 225 & $05^{\prime}$ & RU3 \\
\hline ATOM & 226 & C5 ' & RU3 \\
\hline ATOM & 227 & H5 ' 1 & RU3 \\
\hline ATOM & 228 & H5 ' 2 & RU3 \\
\hline ATOM & 229 & C4' & RU3 \\
\hline ATOM & 230 & $\mathrm{H} 4^{\prime}$ & RU3 \\
\hline ATOM & 231 & O4' & RU3 \\
\hline ATOM & 232 & $\mathrm{C} 1{ }^{\prime}$ & RU3 \\
\hline ATOM & 233 & H1' & RU3 \\
\hline ATOM & 234 & N1 & RU3 \\
\hline ATOM & 235 & C6 & RU3 \\
\hline ATOM & 236 & H 6 & RU3 \\
\hline ATOM & 237 & C5 & RU3 \\
\hline ATOM & 238 & H5 & RU3 \\
\hline ATOM & 239 & C4 & RU3 \\
\hline ATOM & 240 & 04 & RU3 \\
\hline ATOM & 241 & N3 & RU3 \\
\hline ATOM & 242 & H3 & RU3 \\
\hline ATOM & 243 & $\mathrm{C} 2$ & RU3 \\
\hline ATOM & 244 & $\mathrm{O} 2$ & RU3 \\
\hline ATOM & 245 & C3' & RU3 \\
\hline ATOM & 246 & H3 ' & RU3 \\
\hline ATOM & 247 & C2 ${ }^{\prime}$ & RU3 \\
\hline ATOM & 248 & H2'1 & RU3 \\
\hline ATOM & 249 & $02^{\prime}$ & RU3 \\
\hline ATOM & 250 & HO' 2 & RU3 \\
\hline ATOM & 251 & $03^{\prime}$ & RU3 \\
\hline ATOM & 252 & H3 T & RU3 \\
\hline
\end{tabular}

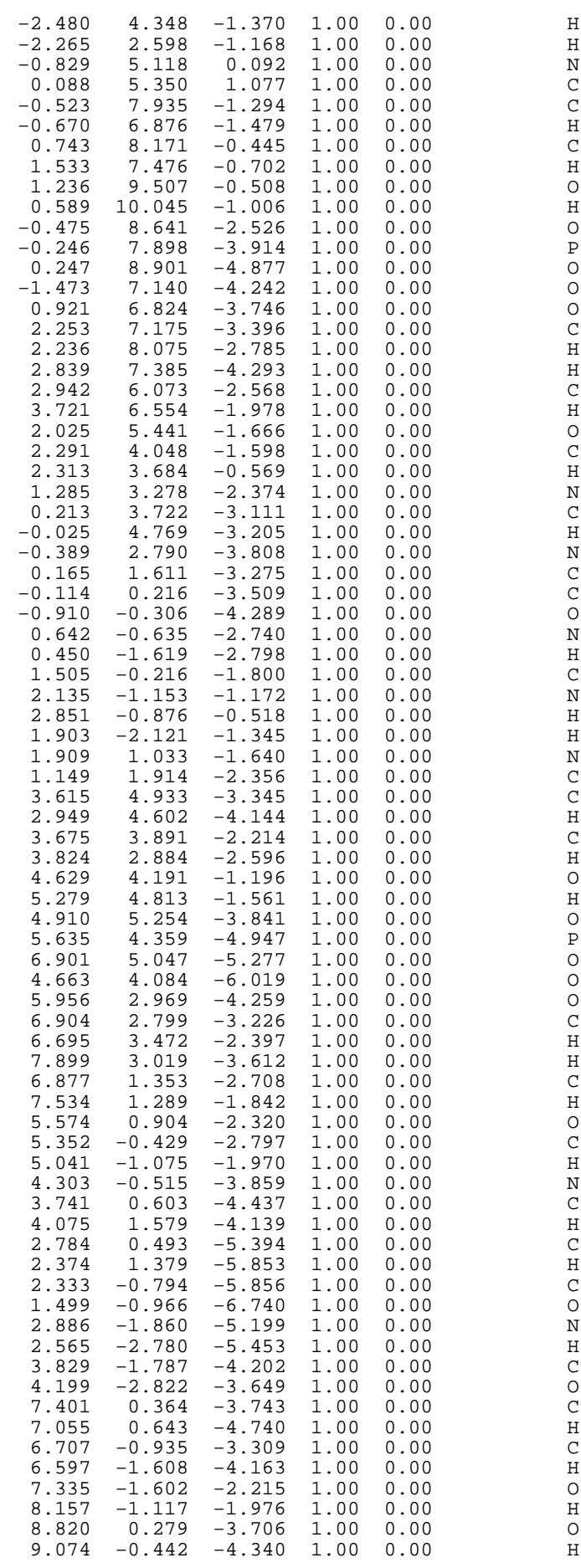

A-80 deg-QM.pdb

\begin{tabular}{|c|c|c|c|}
\hline ATOM & 1 & H5T & RA5 \\
\hline ATOM & 2 & $05^{\prime}$ & RA5 \\
\hline ATOM & 3 & C5' & RA5 \\
\hline ATOM & 4 & H5 ' 1 & RA5 \\
\hline ATOM & 5 & H5 ' 2 & RA5 \\
\hline ATOM & 6 & C4' & RA5 \\
\hline ATOM & 7 & H4 ' & RA5 \\
\hline ATOM & 8 & O4' & RA5 \\
\hline ATOM & 9 & $\mathrm{C} 1^{\prime}$ & RA5 \\
\hline ATOM & 10 & H1 ' & RA5 \\
\hline ATOM & 11 & N9 & RA5 \\
\hline ATOM & 12 & C8 & RA5 \\
\hline ATOM & 13 & $\mathrm{H} 8$ & RA5 \\
\hline ATOM & 14 & N7 & RA5 \\
\hline ATOM & 15 & C5 & RA5 \\
\hline ATOM & 16 & C6 & RA5 \\
\hline ATOM & 17 & N6 & RA5 \\
\hline ATOM & 18 & H61 & RA5 \\
\hline ATOM & 19 & H62 & RA5 \\
\hline ATOM & 20 & $\mathrm{~N} 1$ & RA5 \\
\hline ATOM & 21 & C2 & RA5 \\
\hline ATOM & 22 & $\mathrm{H} 2$ & RA5 \\
\hline ATOM & 23 & N3 & RA5 \\
\hline ATOM & 24 & C4 & RA5 \\
\hline ATOM & 25 & C3' & RA5 \\
\hline ATOM & 26 & H3' & RA5 \\
\hline ATOM & 27 & $C 2^{\prime}$ & RA5 \\
\hline ATOM & 28 & H2' 1 & RA5 \\
\hline ATOM & 29 & 021 & RAS \\
\hline
\end{tabular}

$\begin{array}{rrrrr}-3.939 & -9.767 & -7.120 & 1.00 & 0.00 \\ -4.190 & -9.990 & -6.201 & 1.00 & 0.00 \\ -3.497 & -11.107 & -5.710 & 1.00 & 0.00 \\ -3.209 & -11.793 & -6.511 & 1.00 & 0.00 \\ -4.167 & -11.665 & -5.055 & 1.00 & 0.00 \\ -2.269 & -10.748 & -4.907 & 1.00 & 0.00 \\ -1.825 & -11.689 & -4.557 & 1.00 & 0.00 \\ -1.328 & -10.044 & -5.733 & 1.00 & 0.00 \\ -0.603 & -9.103 & -4.990 & 1.00 & 0.00 \\ 0.473 & -9.259 & -5.108 & 1.00 & 0.00 \\ -0.889 & -7.758 & -5.491 & 1.00 & 0.00 \\ -1.909 & -7.366 & -6.298 & 1.00 & 0.00 \\ -2.691 & -8.042 & -6.603 & 1.00 & 0.00 \\ -1.843 & -6.105 & -6.635 & 1.00 & 0.00 \\ -0.712 & -5.633 & -6.011 & 1.00 & 0.00 \\ -0.082 & -4.368 & -5.982 & 1.00 & 0.00 \\ -0.581 & -3.289 & -6.558 & 1.00 & 0.00 \\ -0.068 & -2.415 & -6.542 & 1.00 & 0.00 \\ -1.518 & -3.295 & -6.941 & 1.00 & 0.00 \\ 1.077 & -4.279 & -5.309 & 1.00 & 0.00 \\ 1.538 & -5.333 & -4.652 & 1.00 & 0.00 \\ 2.461 & -5.175 & -4.109 & 1.00 & 0.00 \\ 1.006 & -6.543 & -4.566 & 1.00 & 0.00 \\ -0.104 & -6.650 & -5.297 & 1.00 & 0.00 \\ -2.470 & -9.796 & -3.727 & 1.00 & 0.00 \\ -3.127 & -8.976 & -4.037 & 1.00 & 0.00 \\ -1.047 & -9.277 & -3.537 & 1.00 & 0.00 \\ -0.995 & -8.331 & -3.003 & 1.00 & 0.00 \\ -0.215 & -10.226 & -2.921 & 1.00 & 0.00\end{array}$




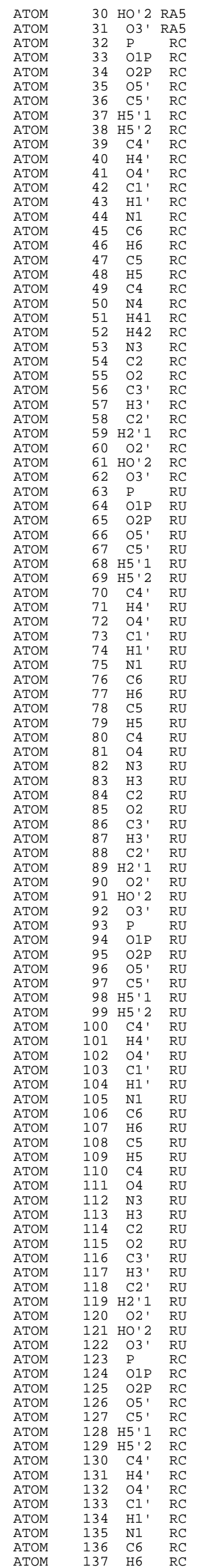

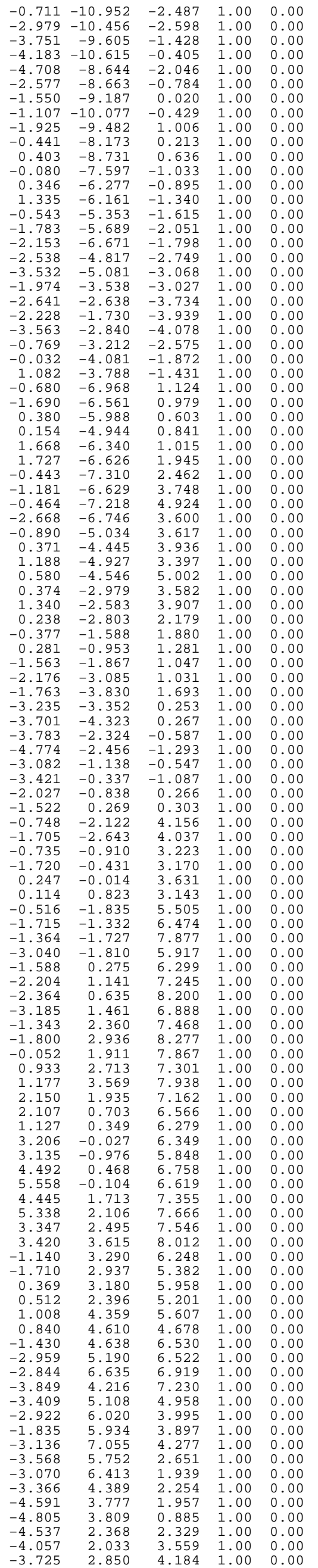




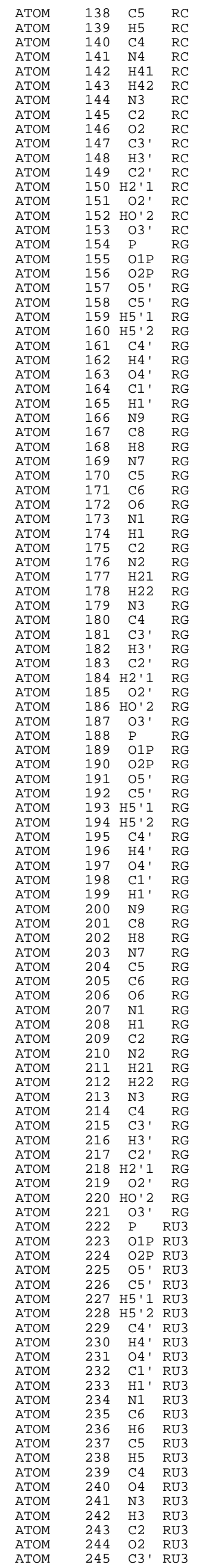

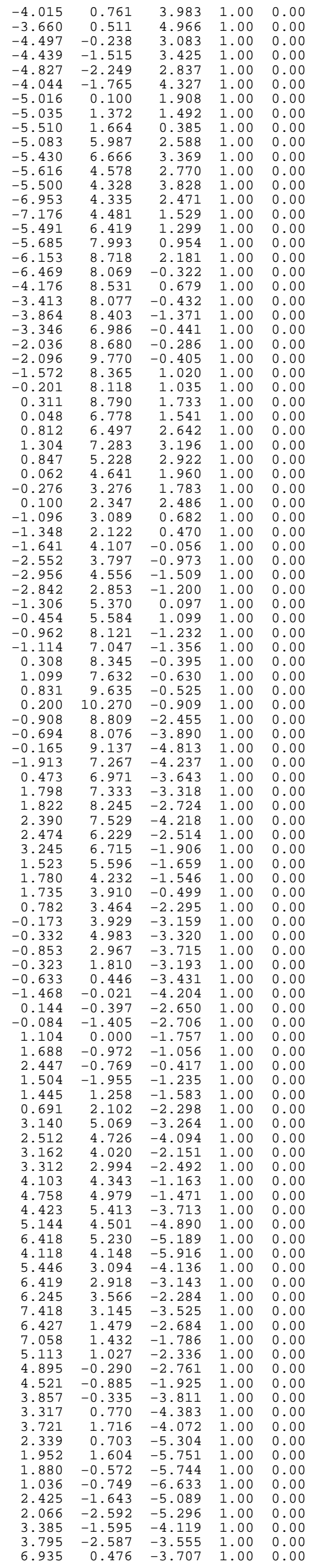




$\begin{array}{llrl}\text { ATOM } & 246 & \text {H}^{\prime} & \text { RU3 } \\ \text { ATOM } & 247 & \text { C2' } & \text { RU3 } \\ \text { ATOM } & 248 & \text { H2'1 } & \text { RU3 } \\ \text { ATOM } & 249 & \text { O2' } & \text { RU3 } \\ \text { ATOM } & 250 & \text { HO'2 } & \text { RU3 } \\ \text { ATOM } & 251 & \text { O3' } & \text { RU3 } \\ \text { ATOM } & 252 & \text { H3T } & \text { RU3 } \\ \text { END } & & & \end{array}$

8
8
8
8
8
8
8

A-180 deg-QM.pdb

ATOM 1 H5T RA5 $\begin{array}{llll}\text { ATOM } & 2 & \text { H5T } & \text { RA5 } \\ & 3 & \text { RA5 }\end{array}$ ATOM 3 C5' RA5 ATOM 4 H5' 1 RA5 ATOM 5 H5'2 RA5 $\begin{array}{llll}\text { ATOM } & 6 & \text { C4 } & \text { RA5 } \\ \text { ATOM } & 7 & \text { H4 } & \text { RA5 }\end{array}$ $\begin{array}{llll}\text { ATOM } & 7 & \text { H4' } & \text { RA5 } \\ \text { ATOM } & 8 & 4^{\prime} & \text { RA5 }\end{array}$ $\begin{array}{llll}\text { ATOM } & 8 & \text { O4' } & \text { RA5 } \\ \text { ATOM } & 9 & \text { C1' } & \text { RA5 }\end{array}$ $\begin{array}{lrlll}\text { ATOM } & 9 & \text { C1' } & \text { RA5 } \\ \text { ATOM } & 10 & \text { H1' } & \text { RA5 }\end{array}$ $\begin{array}{llll}\text { ATOM } & 10 & \text { H1' } & \text { RA5 } \\ \text { ATOM } & 11 & \text { N9 } & \text { RA5 }\end{array}$ $\begin{array}{llll}\text { ATOM } & 11 & \text { N9 } & \text { RA5 } \\ \text { ATOM } & 12 & \text { C8 } & \text { RA5 }\end{array}$ ATOM 13 H8 RA5 $\begin{array}{llll}\text { ATOM } & 14 & \text { N7 } & \text { RA5 } \\ \text { ATOM } & 15 & \text { C5 } & \text { RA5 }\end{array}$ ATOM $\quad 15$ C5 RA5 $\begin{array}{llll}\text { ATOM } & 16 & \text { C6 } & \text { RA5 } \\ & 17 & & \end{array}$ ATOM 17 N6 RA5 $\begin{array}{llll}\text { ATOM } & 18 & \text { H61 } & \text { RA5 } \\ \text { ATOM } & 19 & \text { H62 } & \text { RA5 }\end{array}$ $\begin{array}{llll}\text { ATOM } & 19 & \text { H62 } & \text { RA5 } \\ \text { ATOM } & 20 & \text { N1 } & \text { RA5 }\end{array}$ $\begin{array}{llll}\text { ATOM } & 20 & \text { N1 } & \text { RA5 } \\ \text { ATOM } & 21 & \text { C2 } & \text { RA5 }\end{array}$ $\begin{array}{llll}\text { ATOM } & 21 & \text { C2 } & \text { RA5 } \\ \text { ATOM } & 22 & \text { H2 } & \text { RA5 }\end{array}$ ATOM 23 N3 RA5 ATOM 24 C4 RA5 ATOM 25 C3' RA5 ATOM 26 H3' RA5 ATOM 27 C2' RA5 ATOM 28 H2' 1 RA5 ATOM 29 O2' RA5 ATOM 30 HO'2 RA5 ATOM 31 O3' RA5 $\begin{array}{llll}\text { ATOM } & 32 & \mathrm{P} & \mathrm{RC} \\ & 33 & \mathrm{O} 1 \mathrm{P} & \mathrm{RC}\end{array}$ $\begin{array}{llll}\text { ATOM } & 33 & 01 P & \text { RC } \\ \text { ATOM } & 34 & \text { O2P } & \text { RC }\end{array}$ $\begin{array}{llll}\text { ATOM } & 34 & \text { O2P } & \text { RC } \\ \text { ATOM } & 35 & 051 & \text { RC }\end{array}$ $\begin{array}{llll}\text { ATOM } & 35 & 0^{\prime} & \text { RC } \\ \text { ATOM } & 36 & \text { C5 }^{\prime} & \text { RC }\end{array}$ $\begin{array}{llll}\text { ATOM } & 36 & \text { C5 }^{\prime} & \text { RC } \\ \text { ATOM } & 37 & \mathrm{H}^{\prime} 1 & \mathrm{RC}\end{array}$ ATOM 38 H5'2 RC ATOM $\quad 39$ C4' RC ATOM $40 \quad \mathrm{H}^{\prime}{ }^{\prime} \quad \mathrm{RC}$ $\begin{array}{llll}\text { ATOM } & 41 & 04{ }^{\prime} & \text { RC } \\ \text { ATOM } & 42 & \text { C1 }^{\prime} & \text { RC }\end{array}$ ATOM 43 H1' RC $\begin{array}{llll}\text { ATOM } & 44 & \text { N1 } & \text { RC } \\ \text { ATOM } & 45 & \text { C6 } & \text { RC }\end{array}$ $\begin{array}{llll}\text { ATOM } & 46 & \mathrm{H} 6 & \mathrm{RC}\end{array}$ ATOM 47 C5 $\mathrm{RC}$ ATOM 48 H5 $\quad$ RC $\begin{array}{llll}\text { ATOM } & 49 & \mathrm{C} 4 & \mathrm{RC}\end{array}$ $\begin{array}{llll}\text { ATOM } & 50 & \text { N4 } & \text { RC } \\ \text { ATOM } & 51 & \text { H41 } & \text { RC }\end{array}$ $\begin{array}{llll}\text { ATOM } & 52 & \text { H42 } & \text { RC }\end{array}$ $\begin{array}{llll}\text { ATOM } & 53 & \text { N3 } & \text { RC } \\ \text { ATOM } & 54 & \text { C2 } & \text { RC }\end{array}$ $\begin{array}{llll}\text { ATOM } & 54 & \text { C2 } & \text { RC } \\ \text { ATOM } & 55 & \text { O2 } & \text { RC }\end{array}$ ATOM $56 \mathrm{C}^{\prime}$ RC ATOM $\quad 57 \quad \mathrm{H}^{\prime}{ }^{\prime} \quad \mathrm{RC}$ ATOM $\quad 58$ C2' RC $\begin{array}{llll}\text { ATOM } & 59 & \text { H2' } 1 & \text { RC }\end{array}$ ATOM $61 \mathrm{HO}^{\prime 2} \mathrm{RC}$ ATOM $62 \mathrm{O}^{\prime} \mathrm{RC}$ ATOM $\quad 63 \quad \mathrm{P} \quad \mathrm{RU}$ ATOM 64 O1P RU $\begin{array}{llll}\text { ATOM } & 65 & \mathrm{O} 2 \mathrm{P} & \mathrm{RU}\end{array}$ ATOM 66 O5' RU ATOM 67 C5' RU $\begin{array}{llll}\text { ATOM } & 69 & \mathrm{H} 5.2 & \mathrm{RU}\end{array}$ ATOM 70 C4' RU ATOM $\quad 71$ H4' RU $\begin{array}{llll}\text { ATOM } & 72 & 04^{\prime} & \mathrm{RU}\end{array}$ $\begin{array}{llll}\text { ATOM } & 73 & \text { C1' } & \text { RU } \\ \text { ATOM } & 74 & \text { H1 } & \text { RU }\end{array}$ ATOM $\quad 75$ N1 RU $\begin{array}{llll}\text { ATOM } & 76 & \text { C6 } & \text { RU } \\ & 77 & \text { H6 } & \text { RU }\end{array}$ $\begin{array}{llll}\text { ATOM } & 78 & \mathrm{C} 5 & \mathrm{RU} \\ & 79 & \mathrm{H} & \mathrm{RU}\end{array}$ ATOM 79 H5 RU $\begin{array}{llll}\text { ATOM } & 80 & \mathrm{C} 4 & \mathrm{RU}\end{array}$ $\begin{array}{llll}\text { ATOM } & 81 & 04 & \mathrm{RU}\end{array}$ $\begin{array}{llll}\text { ATOM } & 82 & \mathrm{~N} 3 & \mathrm{RU}\end{array}$ ATOM 83 H3 RU $\begin{array}{llll}\text { ATOM } & 84 & \text { C2 } & \text { RU } \\ \text { ATOM } & 85 & \text { O2 } & \text { RU }\end{array}$ ATOM 86 C3' RU ATOM $\quad 87$ H3' RU $\begin{array}{llll}A T O M & 88 & \mathrm{C}^{\prime} & \mathrm{RU}\end{array}$ $\begin{array}{llll}\text { ATOM } & 89 & \text { H2' } & \text { RU } \\ \text { ATOM } & 90 & 02 & \text { RU }\end{array}$ ATOM $91 \mathrm{HO}^{\prime 2} \mathrm{RU}$ ATOM $92 \mathrm{O}^{\prime}$ RU $\begin{array}{llll}\text { ATOM } & 93 & \mathrm{P} & \mathrm{RU} \\ & 94 & \mathrm{O} 1 \mathrm{P} & \mathrm{RU}\end{array}$ $\begin{array}{llll}\text { ATOM } & 94 & \text { O1P } & \text { RU } \\ \text { ATOM } & 95 & \text { O2P } & \text { RU }\end{array}$ ATOM 96 O5' RU $\begin{array}{llll}\text { ATOM } & 97 & \text { C5 }^{\prime} & \text { RU } \\ \text { ATOM } & 98 & \text { H5' } 1 & \text { RU }\end{array}$

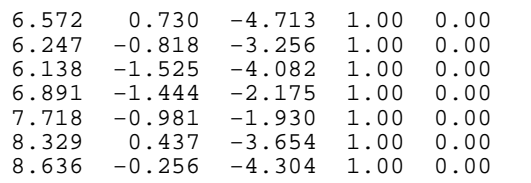

$\begin{array}{llllll}-3.908 & -9.768 & -7.114 & 1.00 & 0.00\end{array}$ $\begin{array}{lllll}-4.157 & -9.994 & -6.195 & 1.00 & 0.00\end{array}$ $\begin{array}{lllll}-3.456 & -11.106 & -5.705 & 1.00 & 0.00\end{array}$ $\begin{array}{llll}-3.163-11.788 & -6.508 & 1.00 & 0.00\end{array}$ $\begin{array}{llll}-4.121-11.668 & -5.050-1.00 & 0.00\end{array}$ $-2.231-10.735-4.899 \quad 1.000 .00$ $\begin{array}{lllll}-1.779 & -11.673 & -4.549 & 1.00 & 0.00\end{array}$ $\begin{array}{lllll}-1.779 & -11.673 & -4.549 & 1.00 & 0.00\end{array}$ $\begin{array}{lllll}-0.576 & -9.082 & -4.984 & 1.00 & 0.00\end{array}$ $\begin{array}{lllll}0.500 & -9.232 & -5.099 & 1.00 & 0.00\end{array}$ $\begin{array}{lllll}-0.865 & -7.741 & -5.487 & 1.00 & 0.00\end{array}$ $\begin{array}{lllll}-1.885 & -7.354 & -6.295 & 1.00 & 0.00\end{array}$ $\begin{array}{lllll}-2.665 & -8.030 & -6.598 & 1.00 & 0.00\end{array}$ $\begin{array}{lllll}-1.821 & -6.094 & -6.633 & 1.00 & 0.00\end{array}$ $\begin{array}{lllll}-0.694 & -5.619 & -6.011 & 1.00 & 0.00\end{array}$ $\begin{array}{lllll}-0.072 & -4.351 & -5.983 & 1.00 & 0.00\end{array}$ $\begin{array}{lllll}-0.573 & -3.281 & -6.573 & 1.00 & 0.00\end{array}$ $\begin{array}{lllll}-0.068 & -2.403 & -6.560 & 1.00 & 0.00\end{array}$ $\begin{array}{lllll}-1.511 & -3.289 & -6.952 & 1.00 & 0.00\end{array}$ $\begin{array}{rrrrr}-1.511 & -3.289 & -6.952 & 1.00 & 0.00 \\ 1.085 & -4.254 & -5.315 & 1.00 & 0.00\end{array}$ $\begin{array}{lllll}1.085 & -4.254 & -5.315 & 1.00 & 0.00\end{array}$ $\begin{array}{lllll}1.551 & -5.300 & -4.651 & 1.00 & 0.00\end{array}$

$\begin{array}{lllll}1.022 & -6.513 & -4.558 & 1.00 & 0.00\end{array}$ $\begin{array}{lllll}-0.087 & -6.628 & -5.289 & 1.00 & 0.00\end{array}$ $\begin{array}{lllll}-2.439 & -9.783 & -3.725 & 1.00 & 0.00\end{array}$ $\begin{array}{lllll}-3.098 & -8.966 & -4.034 & 1.00 & 0.00\end{array}$ $\begin{array}{lllll}-1.018 & -9.258 & -3.531 & 1.00 & 0.00\end{array}$ $\begin{array}{lllll}-0.972 & -8.313 & -2.995 & 1.00 & 0.00\end{array}$ $\begin{array}{lllll}-0.182 & -10.206 & -2.915 & 1.00 & 0.00\end{array}$ $\begin{array}{lllll}-0.675 & -10.937 & -2.481 & 1.00 & 0.00\end{array}$ $\begin{array}{lllll}-2.949 & -10.447 & -2.595 & 1.00 & 0.00\end{array}$ $\begin{array}{lllll}-3.731 & -9.603 & -1.428 & 1.00 & 0.00\end{array}$ $\begin{array}{lllll}-4.157 & -10.620 & -0.409 & 1.00 & 0.00\end{array}$ $\begin{array}{lllll}-4.694 & -8.649 & -2.047 & 1.00 & 0.00\end{array}$ $\begin{array}{lllll}-2.567 & -8.649 & -0.782 & 1.00 & 0.00\end{array}$ $\begin{array}{lllll}-1.531 & -9.170 & 0.022 & 1.00 & 0.00\end{array}$ $\begin{array}{lllll}-1.083 & -10.056 & -0.431 & 1.00 & 0.00\end{array}$ $\begin{array}{lllll}-1.902 & -9.471 & 1.006 & 1.00 & 0.00\end{array}$ $\begin{array}{lllll}-0.436 & -8.140 & 0.215 & 1.00 & 0.00\end{array}$ $\begin{array}{lllll}0.416 & -8.687 & 0.637 & 1.00 & 0.00\end{array}$ $\begin{array}{lllll}-0.085 & -7.558 & -1.030 & 1.00 & 0.00\end{array}$ $\begin{array}{lllll}0.331 & -6.236 & -0.890 & 1.00 & 0.00\end{array}$ $\begin{array}{lllll}1.323 & -6.112 & -1.322 & 1.00 & 0.00\end{array}$ $\begin{array}{lllll}-0.555 & -5.320 & -1.619 & 1.00 & 0.00\end{array}$ $\begin{array}{lllll}-1.800 & -5.655 & -2.041 & 1.00 & 0.00\end{array}$ $\begin{array}{lllll}-2.175 & -6.633 & -1.780 & 1.00 & 0.00\end{array}$ $\begin{array}{lllll}-2.556 & -4.789 & -2.744 & 1.00 & 0.00\end{array}$ $\begin{array}{lllll}-3.555 & -5.049 & -3.055 & 1.00 & 0.00\end{array}$ $\begin{array}{lllll}-1.985 & -3.519 & -3.040 & 1.00 & 0.00\end{array}$ $\begin{array}{lllll}-2.650 & -2.628 & -3.757 & 1.00 & 0.00\end{array}$ $\begin{array}{lllll}-2.233 & -1.722 & -3.961 & 1.00 & 0.00\end{array}$ $\begin{array}{lllll}-3.576 & -2.822 & -4.092 & 1.00 & 0.00\end{array}$ $\begin{array}{llllll}-0.779 & -3.189 & -2.596 & 1.00 & 0.00\end{array}$ $\begin{array}{lllll}-0.043 & -4.051 & -1.885 & 1.00 & 0.00\end{array}$ $\begin{array}{lllll}1.072 & -3.756 & -1.449 & 1.00 & 0.00\end{array}$ $\begin{array}{lllll}-0.693 & -6.943 & 1.125 & 1.00 & 0.00 \\ -1.706 & -6.550 & 0.982 & 1.00 & 0.00\end{array}$ $\begin{array}{rrrrr}-1.706 & -6.550 & 0.982 & 1.00 & 0.00 \\ 0.353 & -5.947 & 0.610 & 1.00 & 0.00 \\ 0.109 & -4.908 & 0.846 & 1.00 & 0.00\end{array}$ $\begin{array}{lllll}0.109 & -4.908 & 0.846 & 1.00 & 0.00\end{array}$ $\begin{array}{lllll}1.646 & -6.280 & 1.027 & 1.00 & 0.00\end{array}$ $\begin{array}{lllll}1.708 & -6.581 & 1.954 & 1.00 & 0.00\end{array}$ $\begin{array}{lllll}-0.456 & -7.306 & 2.464 & 1.00 & 0.00\end{array}$ $\begin{array}{lllll}-1.189 & -6.632 & 3.751 & 1.00 & 0.00\end{array}$ $\begin{array}{lllll}-0.458 & -7.208 & 4.925 & 1.00 & 0.00\end{array}$ $\begin{array}{lllll}-2.677 & -6.765 & 3.622 & 1.00 & 0.00\end{array}$ $\begin{array}{lllll}-2.677 & -6.765 & 3.622 & 1.00 & 0.00 \\ -0.919 & -5.037 & 3.605 & 1.00 & 0.00\end{array}$ $\begin{array}{lllll}0.324 & -4.414 & 3.943 & 1.00 & 0.00\end{array}$ $\begin{array}{lllll}0.324 & -4.414 & 3.943 & 1.00 & 0.00\end{array}$ $\begin{array}{lllll}1.156 & -4.839 & 3.377 & 1.00 & 0.00\end{array}$ $\begin{array}{lllll}0.544 & -4.552 & 5.002 & 1.00 & 0.00 \\ 0.237 & -2.935 & 3.655 & 1.00 & 0.00\end{array}$ $\begin{array}{lllll}0.237 & -2.935 & 3.655 & 1.00 & 0.00 \\ 1.149 & -2.472 & 4.041 & 1.00 & 0.00\end{array}$ $\begin{array}{lllll}0.137 & -2.705 & 2.261 & 1.00 & 0.00\end{array}$ $\begin{array}{lllll}-0.542 & -1.517 & 2.000 & 1.00 & 0.00\end{array}$ $\begin{array}{lllll}0.098 & -0.771 & 1.523 & 1.00 & 0.00\end{array}$ $\begin{array}{lllll}-1.629 & -1.823 & 1.058 & 1.00 & 0.00\end{array}$ $\begin{array}{lllll}-2.254 & -3.033 & 1.071 & 1.00 & 0.00\end{array}$ $\begin{array}{lllll}-1.866 & -3.758 & 1.768 & 1.00 & 0.00\end{array}$ $\begin{array}{lllll}-3.291 & -3.328 & 0.272 & 1.00 & 0.00\end{array}$ $\begin{array}{lllll}-3.756 & -4.299 & 0.303 & 1.00 & 0.00\end{array}$ $\begin{array}{lllll}-3.814 & -2.326 & -0.615 & 1.00 & 0.00\end{array}$ $\begin{array}{lllll}-4.797 & -2.476 & -1.327 & 1.00 & 0.00\end{array}$ $\begin{array}{lllll}-3.093 & -1.152 & -0.612 & 1.00 & 0.00 \\ -3.411 & -0.369 & -1.189 & 1.00 & 0.00\end{array}$ $\begin{array}{rrrrr}-3.411 & -0.369 & -1.189 & 1.00 & 0.00 \\ -2.044 & -0.826 & 0.205 & 1.00 & 0.00\end{array}$ $\begin{array}{rrrrr}-2.044 & -0.826 & 0.205 & 1.00 & 0.00 \\ -1.518 & 0.267 & 0.183 & 1.00 & 0.00\end{array}$ $\begin{array}{rrrrr}-1.518 & 0.267 & 0.183 & 1.00 & 0.00 \\ -0.968 & -2.215 & 4.238 & 1.00 & 0.00\end{array}$ $\begin{array}{lllll}-0.968 & -2.215 & 4.238 & 1.00 & 0.00 \\ -1.875 & -2.817 & 4.125 & 1.00 & 0.00\end{array}$ $\begin{array}{lllll}-1.076 & -0.978 & 3.337 & 1.00 & 0.00\end{array}$ $\begin{array}{lllll}-2.110 & -0.638 & 3.238 & 1.00 & 0.00\end{array}$ $\begin{array}{lllll}-0.229 & 0.036 & 3.768 & 1.00 & 0.00\end{array}$ $\begin{array}{lllll}-0.587 & 0.382 & 4.591 & 1.00 & 0.00\end{array}$ $\begin{array}{lllll}-0.720 & -1.934 & 5.598 & 1.00 & 0.00\end{array}$ $\begin{array}{lllll}-1.882 & -1.372 & 6.571 & 1.00 & 0.00\end{array}$ $\begin{array}{lllll}-1.490 & -1.669 & 7.985 & 1.00 & 0.00\end{array}$ $\begin{array}{lllll}-3.222 & -1.857 & 6.068 & 1.00 & 0.00\end{array}$ $\begin{array}{rrrrr}-3.222 & -1.857 & 6.068 & 1.00 & 0.00 \\ -1.718 & 0.227 & 6.279 & 1.00 & 0.00\end{array}$ $\begin{array}{lllll}-2.217 & 1.189 & 7.209 & 1.00 & 0.00\end{array}$ $8.187 \quad 1.00 \quad 0.00$ 


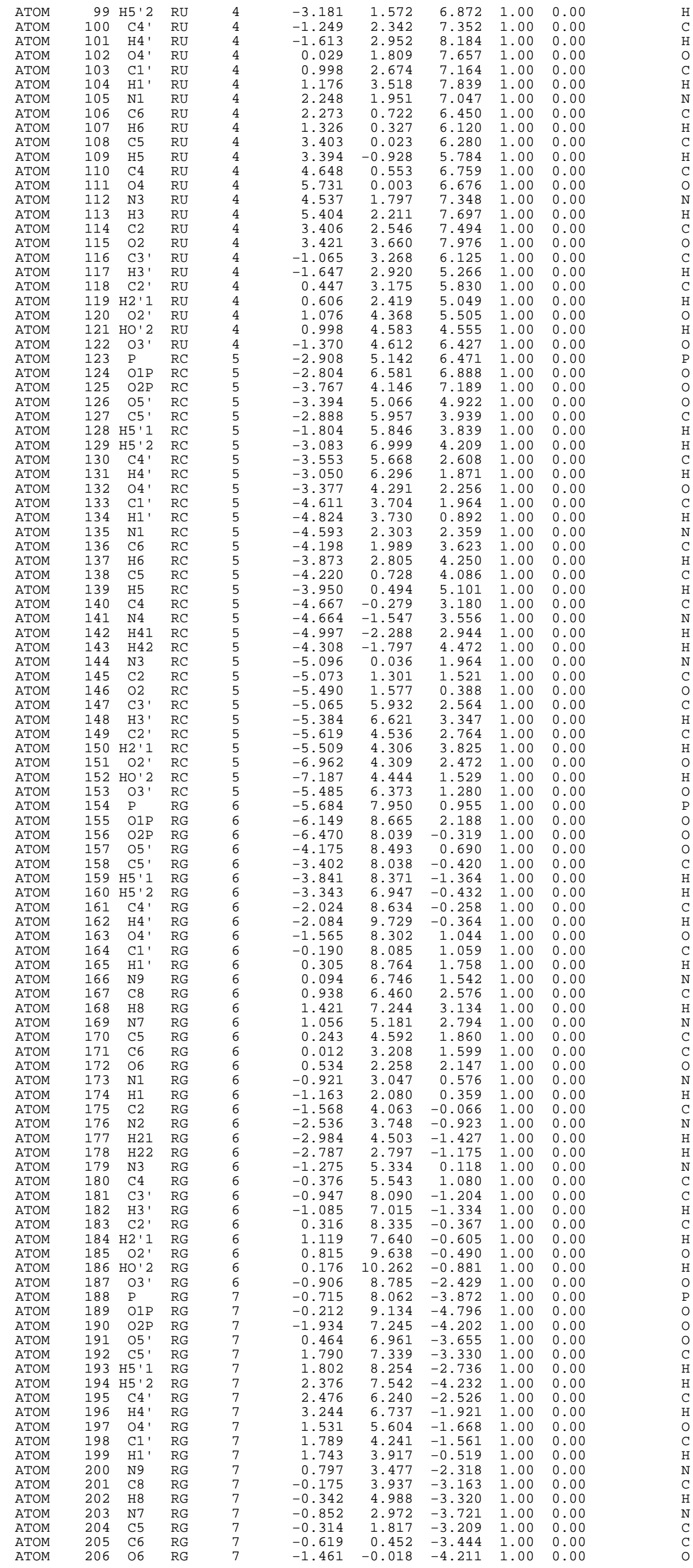




$\begin{array}{llll}\text { ATOM } & 207 & \text { N1 } & \text { RG } \\ \text { ATOM } & 208 & \text { H1 } & \text { RG } \\ \text { ATOM } & 209 & \text { C2 } & \text { RG } \\ \text { ATOM } & 210 & \text { N2 } & \text { RG } \\ \text { ATOM } & 211 & \text { H21 } & \text { RG } \\ \text { ATOM } & 212 & \text { H22 } & \text { RG } \\ \text { ATOM } & 213 & \text { N3 } & \text { RG } \\ \text { ATOM } & 214 & \text { C4 } & \text { RG } \\ \text { ATOM } & 215 & \text { C3' } & \text { RG } \\ \text { ATOM } & 216 & \text { H3' } & \text { RG } \\ \text { ATOM } & 217 & \text { C2' } & \text { RG } \\ \text { ATOM } & 218 & \text { H2'1 } & \text { RG } \\ \text { ATOM } & 219 & \text { O2' } & \text { RG } \\ \text { ATOM } & 220 & \text { HO'2 } & \text { RG } \\ \text { ATOM } & 221 & \text { O3' } & \text { RG } \\ \text { ATOM } & 222 & \text { P } & \text { RU3 } \\ \text { ATOM } & 223 & \text { O1P } & \text { RU3 } \\ \text { ATOM } & 224 & \text { O2P } & \text { RU3 } \\ \text { ATOM } & 225 & \text { O5' } & \text { RU3 } \\ \text { ATOM } & 226 & \text { C5' } & \text { RU3 } \\ \text { ATOM } & 227 & \text { H5'1 } & \text { RU3 } \\ \text { ATOM } & 228 & \text { H5'2 } & \text { RU3 } \\ \text { ATOM } & 229 & \text { C4' } & \text { RU3 } \\ \text { ATOM } & 230 & \text { H4' } & \text { RU3 } \\ \text { ATOM } & 231 & \text { O4' } & \text { RU3 } \\ \text { ATOM } & 232 & \text { C1'' } & \text { RU3 } \\ \text { ATOM } & 233 & \text { H1' } & \text { RU3 } \\ \text { ATOM } & 234 & \text { N1 } & \text { RU3 } \\ \text { ATOM } & 235 & \text { C6 } & \text { RU3 } \\ \text { ATOM } & 236 & \text { H6 } & \text { RU3 } \\ \text { ATOM } & 237 & \text { C5 } & \text { RU3 } \\ \text { ATOM } & 238 & \text { H5 } & \text { RU3 } \\ \text { ATOM } & 239 & \text { C4 } & \text { RU3 } \\ \text { ATOM } & 240 & \text { O4 } & \text { RU3 } \\ \text { ATOM } & 241 & \text { N3 } & \text { RU3 } \\ \text { ATOM } & 242 & \text { H3 } & \text { RU3 } \\ \text { ATOM } & 243 & \text { C2 } & \text { RU3 } \\ \text { ATOM } & 244 & \text { O2 } & \text { RU3 } \\ \text { ATOM } & 245 & \text { C3' } & \text { RU3 } \\ \text { ATOM } & 246 & \text { H3' } & \text { RU3 } \\ \text { ATOM } & 247 & \text { C2' } & \text { RU3 } \\ \text { ATOM } & 248 & \text { H2''1 } & \text { RU3 } \\ \text { ATOM } & 249 & \text { O2' } & \text { RU3 } \\ \text { ATOM } & 250 & \text { HO'2 } & \text { RU3 } \\ \text { ATOM } & 251 & \text { O3' } & \text { RU3 } \\ \text { ATOM } & 252 & \text { H3T } & \text { RU3 } \\ \text { FND } & & & \\ & & & \\ & & \end{array}$

$\begin{array}{rrrrr}0.168 & -0.387 & -2.672 & 1.00 & 0.00 \\ -0.059 & -1.391 & -2.726 & 1.00 & 0.00 \\ 1.132 & 0.016 & -1.785 & 1.00 & 0.00 \\ 1.729 & -0.950 & -1.088 & 1.00 & 0.00 \\ 2.465 & -0.730 & -0.433 & 1.00 & 0.00 \\ 1.527 & -1.934 & -1.246 & 1.00 & 0.00 \\ 1.471 & 1.276 & -1.620 & 1.00 & 0.00 \\ 0.712 & 2.113 & -2.327 & 1.00 & 0.00 \\ 3.143 & 5.082 & -3.273 & 1.00 & 0.00 \\ 2.516 & 4.736 & -4.101 & 1.00 & 0.00 \\ 3.175 & 4.037 & -2.158 & 1.00 & 0.00 \\ 3.324 & 3.013 & -2.498 & 1.00 & 0.00 \\ 4.117 & 4.367 & -1.168 & 1.00 & 0.00 \\ 4.756 & 5.021 & -1.478 & 1.00 & 0.00 \\ 4.426 & 5.436 & -3.729 & 1.00 & 0.00 \\ 5.147 & 4.526 & -4.906 & 1.00 & 0.00 \\ 6.419 & 5.259 & -5.208 & 1.00 & 0.00 \\ 4.122 & 4.170 & -5.932 & 1.00 & 0.00 \\ 5.453 & 3.117 & -4.157 & 1.00 & 0.00 \\ 6.433 & 2.945 & -3.160 & 1.00 & 0.00 \\ 6.254 & 3.595 & -2.303 & 1.00 & 0.00 \\ 7.429 & 3.179 & -3.546 & 1.00 & 0.00 \\ 6.442 & 1.504 & -2.703 & 1.00 & 0.00 \\ 7.080 & 1.459 & -1.812 & 1.00 & 0.00 \\ 5.128 & 1.056 & -2.347 & 1.00 & 0.00 \\ 4.910 & -0.260 & -2.774 & 1.00 & 0.00 \\ 4.532 & -0.856 & -1.942 & 1.00 & 0.00 \\ 3.873 & -0.306 & -3.826 & 1.00 & 0.00 \\ 3.333 & 0.797 & -4.401 & 1.00 & 0.00 \\ 3.740 & 1.744 & -4.099 & 1.00 & 0.00 \\ 2.352 & 0.730 & -5.322 & 1.00 & 0.00 \\ 1.964 & 1.631 & -5.769 & 1.00 & 0.00 \\ 1.887 & -0.546 & -5.757 & 1.00 & 0.00 \\ 1.040 & -0.724 & -6.641 & 1.00 & 0.00 \\ 2.435 & -1.615 & -5.099 & 1.00 & 0.00 \\ 2.072 & -2.562 & -5.301 & 1.00 & 0.00 \\ 3.400 & -1.567 & -4.132 & 1.00 & 0.00 \\ 3.812 & -2.561 & -3.571 & 1.00 & 0.00 \\ 6.946 & 0.500 & -3.725 & 1.00 & 0.00 \\ 6.580 & 0.751 & -4.729 & 1.00 & 0.00 \\ 6.260 & -0.790 & -3.267 & 1.00 & 0.00 \\ 6.151 & -1.501 & -4.089 & 1.00 & 0.00 \\ 6.903 & -1.406 & -2.180 & 1.00 & 0.00 \\ 7.735 & -0.947 & -1.937 & 1.00 & 0.00 \\ 8.340 & 0.464 & -3.675 & 1.00 & 0.00 \\ 8.644 & -0.231 & -4.323 & 1.00 & 0.00\end{array}$

B-80 deg-MM.pdb

\begin{tabular}{|c|c|c|c|}
\hline ATOM & 1 & $\mathrm{H} 5 \mathrm{~T}$ & RA5 \\
\hline ATOM & 2 & O5' & RA5 \\
\hline ATOM & 3 & C5 ${ }^{\prime}$ & RA5 \\
\hline ATOM & 4 & H5 ' 1 & RA5 \\
\hline ATOM & 5 & H5 ' 2 & RA5 \\
\hline ATOM & 6 & C4' & RA5 \\
\hline ATOM & 7 & $\mathrm{H} 4^{\prime}$ & RA5 \\
\hline ATOM & 8 & $04^{\prime}$ & RA5 \\
\hline ATOM & 9 & $\mathrm{C} 11^{\prime}$ & RA5 \\
\hline ATOM & 10 & H1 ' & RA5 \\
\hline ATOM & 11 & N9 & RA5 \\
\hline ATOM & 12 & C8 & RA5 \\
\hline ATOM & 13 & H8 & RA5 \\
\hline ATOM & 14 & N7 & RA5 \\
\hline ATOM & 15 & C5 & RA5 \\
\hline ATOM & 16 & C6 & RA5 \\
\hline ATOM & 17 & N6 & RA5 \\
\hline ATOM & 18 & H 61 & RA5 \\
\hline ATOM & 19 & H 62 & RA5 \\
\hline ATOM & 20 & N1 & RA5 \\
\hline ATOM & 21 & C2 & RA5 \\
\hline ATOM & 22 & H2 & RA5 \\
\hline ATOM & 23 & N3 & RA5 \\
\hline ATOM & 24 & C4 & RA5 \\
\hline ATOM & 25 & C3' & RA5 \\
\hline ATOM & 26 & H3 ${ }^{\prime}$ & RA5 \\
\hline ATOM & 27 & C2 ${ }^{\prime}$ & RA5 \\
\hline ATOM & 28 & H2' 1 & RA5 \\
\hline ATOM & 29 & $02^{\prime}$ & RA5 \\
\hline ATOM & 30 & HO' 2 & RA5 \\
\hline ATOM & 31 & O3' & RA5 \\
\hline ATOM & 32 & P & $\mathrm{RC}$ \\
\hline ATOM & 33 & O1P & $\mathrm{RC}$ \\
\hline ATOM & 34 & $\mathrm{O} 2 \mathrm{P}$ & $\mathrm{RC}$ \\
\hline ATOM & 35 & O5' & $\mathrm{RC}$ \\
\hline ATOM & 36 & C5 ${ }^{\prime}$ & $\mathrm{RC}$ \\
\hline ATOM & 37 & H5' 1 & $\mathrm{RC}$ \\
\hline ATOM & 38 & H5 ' 2 & $\mathrm{RC}$ \\
\hline ATOM & 39 & C4 ${ }^{\prime}$ & $\mathrm{RC}$ \\
\hline ATOM & 40 & H4 ' & $\mathrm{RC}$ \\
\hline ATOM & 41 & $04^{\prime}$ & $\mathrm{RC}$ \\
\hline ATOM & 42 & $\mathrm{C} 11^{\prime}$ & $\mathrm{RC}$ \\
\hline ATOM & 43 & H1 ' & $\mathrm{RC}$ \\
\hline ATOM & 44 & N1 & $\mathrm{RC}$ \\
\hline ATOM & 45 & C6 & $\mathrm{RC}$ \\
\hline ATOM & 46 & H 6 & $\mathrm{RC}$ \\
\hline ATOM & 47 & C5 & $\mathrm{RC}$ \\
\hline ATOM & 48 & H5 & $\mathrm{RC}$ \\
\hline ATOM & 49 & C4 & $\mathrm{RC}$ \\
\hline ATOM & 50 & N4 & $\mathrm{RC}$ \\
\hline ATOM & 51 & H 41 & $\mathrm{RC}$ \\
\hline ATOM & 52 & H 42 & $\mathrm{RC}$ \\
\hline ATOM & 53 & N3 & $\mathrm{RC}$ \\
\hline ATOM & 54 & $\mathrm{C} 2$ & $\mathrm{RC}$ \\
\hline ATOM & 55 & O2 & $\mathrm{RC}$ \\
\hline ATOM & 56 & C3' & $\mathrm{RC}$ \\
\hline ATOM & 57 & H3 ${ }^{\prime}$ & $\mathrm{RC}$ \\
\hline ATOM & 58 & C2' & $\mathrm{RC}$ \\
\hline
\end{tabular}

\begin{tabular}{|c|c|c|c|c|}
\hline 2. & -0.743 & -12.502 & 1.00 & 0 \\
\hline & & -12 & & \\
\hline 4. & 0.482 & -12.817 & 1. & 0.00 \\
\hline 5.3 & 4 & -13.391 & 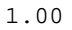 & 0 \\
\hline $3-$ & 0.984 & -13.453 & 1. & 00 \\
\hline & & -11.6 & & \\
\hline 5. & 2.342 & -11.978 & & 00 \\
\hline & & -10.805 & & \\
\hline 5. & 1.191 & -9.446 & 1. & 0.00 \\
\hline 6 . & & -9.04 & & 0 \\
\hline & -0 & -8.6 & & \\
\hline 4. & -1.2 & -9.012 & 1. & 0.00 \\
\hline-4 . & -1 & -9.9 & & \\
\hline & -2. & -8.1 & & 00 \\
\hline & -1 . & -7 & & \\
\hline 5 . & -1 & -5. & & 00 \\
\hline-5 & -2 . & -5 & & \\
\hline & -2.9 & -4.070 & & 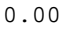 \\
\hline-4 & -3.608 & -5.551 & 1. & .00 \\
\hline-6 . & -0 . & & & \\
\hline 6 . & 0 . & -5 & & \\
\hline & 0. & -4 & & \\
\hline-6 . & & -6 & & 00 \\
\hline . & -0. & -7.3 & & \\
\hline+ & & -10. & & 00 \\
\hline-3 & 0. & -10 & & 0 \\
\hline & 2 . & -9 & & \\
\hline-3 & 1. & -8 . & & 0 \\
\hline & 3.2 & -9.5 & & \\
\hline 4. & 3. & -10 & & \\
\hline-2 . & 2. & -11. & & \\
\hline & & -10 & & 00 \\
\hline-0 . & 3.8 & -11 & & 00 \\
\hline & & -10 & & \\
\hline 1. & 2 . & -8 & & 0 \\
\hline-1 . & 4. & -8 . & & 0 \\
\hline & & & & \\
\hline-0 . & 4. & -8 . & & \\
\hline & 4 . & & & \\
\hline-1 & 5. & -6 & & \\
\hline & 3. & 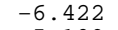 & & \\
\hline-2 & 3 . & -5 . & & 0 \\
\hline-3. & 3. & -4 . & & \\
\hline & & & & \\
\hline-2 . & $0 . \varepsilon=2.8$ & -6 . & & 0 \\
\hline & & & & \\
\hline & -0 & -5 & & \\
\hline & -1 & & & \\
\hline & -0. & -4 & & \\
\hline & -2 . & -4.1 & & 0.00 \\
\hline & & & & \\
\hline & & & & \\
\hline & - & & & \\
\hline & 1 . & & & \\
\hline & 10 & -2 . & & \\
\hline & 3. & -6.2 & & \\
\hline & & & & 0.00 \\
\hline & 3. & -4 . & & \\
\hline- & 2 & -4 & & \\
\hline
\end{tabular}




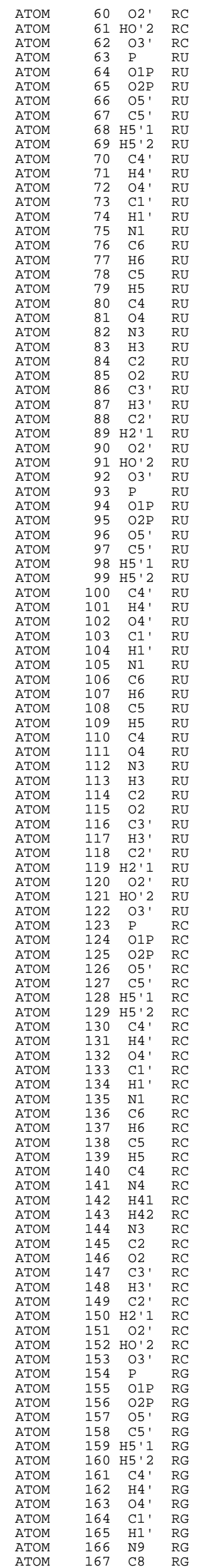

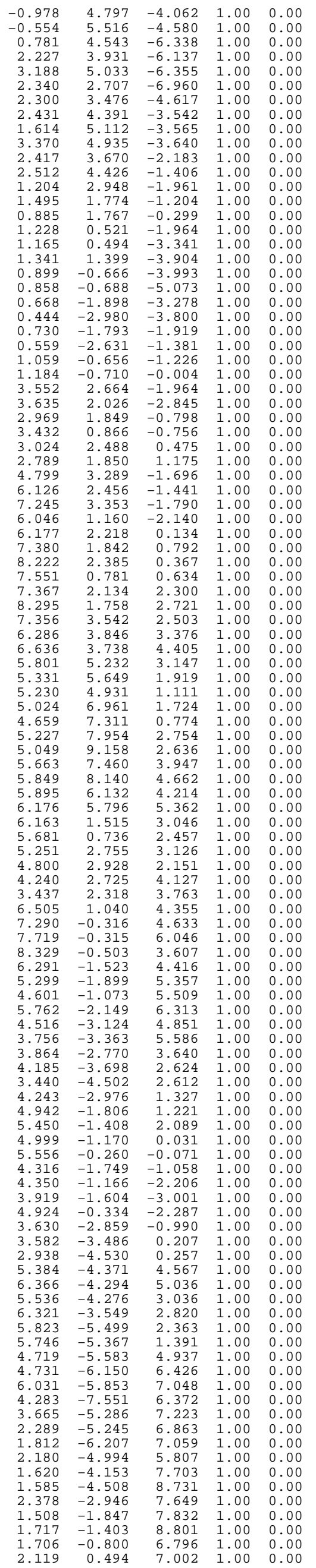




\begin{tabular}{|c|c|c|c|}
\hline ATOM & 168 & H8 & RG \\
\hline ATOM & 169 & N7 & RG \\
\hline ATOM & 170 & C5 & RG \\
\hline ATOM & 171 & C6 & RG \\
\hline ATOM & 172 & 06 & RG \\
\hline ATOM & 173 & N1 & RG \\
\hline АTOM & 174 & H1 & RG \\
\hline ATOM & 175 & $\mathrm{C} 2$ & RG \\
\hline ATOM & 176 & $\mathrm{~N} 2$ & RG \\
\hline ATOM & 177 & H 21 & RG \\
\hline ATOM & 178 & H 22 & RG \\
\hline ATOM & 179 & N3 & RG \\
\hline ATOM & 180 & $\mathrm{C} 4$ & RG \\
\hline ATOM & 181 & C3' & RG \\
\hline ATOM & 182 & H3' & RG \\
\hline ATOM & 183 & C2' & RG \\
\hline ATOM & 184 & $\mathrm{H} 2{ }^{\prime} 1$ & RG \\
\hline ATOM & 185 & $02^{\prime}$ & RG \\
\hline ATOM & 186 & HO' 2 & RG \\
\hline ATOM & 187 & O3' & RG \\
\hline ATOM & 188 & P & RG \\
\hline ATOM & 189 & O1P & RG \\
\hline ATOM & 190 & $\mathrm{O} 2 \mathrm{P}$ & RG \\
\hline ATOM & 191 & $05^{\prime}$ & RG \\
\hline ATOM & 192 & C5' & RG \\
\hline ATOM & 193 & H5' 1 & RG \\
\hline ATOM & 194 & H5' 2 & RG \\
\hline ATOM & 195 & C4 ${ }^{\prime}$ & RG \\
\hline ATOM & 196 & H4 ' & RG \\
\hline АTOM & 197 & O4' & RG \\
\hline ATOM & 198 & $\mathrm{C} 1^{\prime}$ & RG \\
\hline ATOM & 199 & H1 ' & RG \\
\hline ATOM & 200 & N9 & RG \\
\hline ATOM & 201 & C8 & RG \\
\hline ATOM & 202 & H8 & RG \\
\hline ATOM & 203 & N7 & RG \\
\hline ATOM & 204 & C5 & RG \\
\hline ATOM & 205 & C6 & RG \\
\hline АTOM & 206 & 06 & RG \\
\hline ATOM & 207 & N1 & RG \\
\hline ATOM & 208 & $\mathrm{H} 1$ & RG \\
\hline ATOM & 209 & $\mathrm{C} 2$ & RG \\
\hline ATOM & 210 & N2 & RG \\
\hline ATOM & 211 & H 21 & RG \\
\hline ATOM & 212 & H 22 & RG \\
\hline ATOM & 213 & N3 & RG \\
\hline ATOM & 214 & $\mathrm{C} 4$ & RG \\
\hline ATOM & 215 & C3' & RG \\
\hline ATOM & 216 & H3' & RG \\
\hline ATOM & 217 & C2' & RG \\
\hline ATOM & 218 & H2' 1 & RG \\
\hline ATOM & 219 & $02^{\prime}$ & RG \\
\hline ATOM & 220 & $\mathrm{HO}^{\prime} 2$ & RG \\
\hline ATOM & 221 & O3' & RG \\
\hline ATOM & 222 & $\mathrm{P}$ & RU3 \\
\hline ATOM & 223 & O1P & RU3 \\
\hline ATOM & 224 & $\mathrm{O} 2 \mathrm{P}$ & RU3 \\
\hline ATOM & 225 & $05^{\prime}$ & RU3 \\
\hline ATOM & 226 & C5' & RU3 \\
\hline ATOM & 227 & H5' 1 & RU3 \\
\hline ATOM & 228 & H5' 2 & RU3 \\
\hline ATOM & 229 & C4 & RU3 \\
\hline ATOM & 230 & H4 ' & RU3 \\
\hline ATOM & 231 & O4' & RU3 \\
\hline ATOM & 232 & $\mathrm{C} 1^{\prime}$ & RU3 \\
\hline ATOM & 233 & H1 ' & RU3 \\
\hline ATOM & 234 & $\mathrm{~N} 1$ & RU3 \\
\hline ATOM & 235 & $\mathrm{C} 6$ & RU3 \\
\hline ATOM & 236 & H6 & RU3 \\
\hline ATOM & 237 & C5 & RU3 \\
\hline ATOM & 238 & H5 & RU3 \\
\hline ATOM & 239 & $\mathrm{C} 4$ & RU3 \\
\hline ATOM & 240 & 04 & RU3 \\
\hline ATOM & 241 & N3 & RU3 \\
\hline ATOM & 242 & H3 & RU3 \\
\hline ATOM & 243 & $\mathrm{C} 2$ & RU3 \\
\hline ATOM & 244 & 02 & RU3 \\
\hline ATOM & 245 & C3' & RU3 \\
\hline ATOM & 246 & H3' & RU3 \\
\hline ATOM & 247 & C2' & RU3 \\
\hline ATOM & 248 & $\mathrm{H} 2{ }^{\prime} 1$ & RU3 \\
\hline ATOM & 249 & $02^{\prime}$ & RU3 \\
\hline ATOM & 250 & HO' 2 & RU3 \\
\hline ATOM & 251 & O3' & RU3 \\
\hline ATOM & 252 & H3T & RU3 \\
\hline END & & & \\
\hline
\end{tabular}

\begin{tabular}{|c|c|c|c|c|}
\hline 2.337 & 0.887 & 7.987 & 1.00 & 0.00 \\
\hline 2.227 & 1.209 & 5.915 & 1.00 & 0.00 \\
\hline 1.842 & 0.324 & 4.898 & 1.00 & 0.00 \\
\hline 1.730 & 0.491 & 3.469 & 1.00 & 0.00 \\
\hline 1.938 & 1.487 & 2.774 & 1.00 & 0.00 \\
\hline 1.333 & -0.638 & 2.803 & 1.00 & 0.00 \\
\hline 1.248 & -0.611 & 1.803 & 1.00 & 0.00 \\
\hline 1.135 & -1.813 & 3.410 & 1.00 & 0.00 \\
\hline 0.860 & -2.806 & 2.633 & 1.00 & 0.00 \\
\hline 0.690 & -3.683 & 3.110 & 1.00 & 0.00 \\
\hline 0.735 & -2.701 & 1.639 & 1.00 & 0.00 \\
\hline 1.151 & -2.016 & 4.720 & 1.00 & 0.00 \\
\hline 1.543 & -0.914 & 5.427 & 1.00 & 0.00 \\
\hline 0.188 & -3.801 & 7.290 & 1.00 & 0.00 \\
\hline 0.131 & -3.756 & 6.202 & 1.00 & 0.00 \\
\hline 0.078 & -2.379 & 7.880 & 1.00 & 0.00 \\
\hline-0.558 & -1.760 & 7.260 & 1.00 & 0.00 \\
\hline-0.362 & -2.301 & 9.238 & 1.00 & 0.00 \\
\hline 0.156 & -2.935 & 9.782 & 1.00 & 0.00 \\
\hline-0.783 & -4.695 & 7.819 & 1.00 & 0.00 \\
\hline-1.982 & -5.246 & 6.933 & 1.00 & 0.00 \\
\hline-2.932 & -5.917 & 7.843 & 1.00 & 0.00 \\
\hline-1.401 & -6.020 & 5.819 & 1.00 & 0.00 \\
\hline-2.742 & -4.006 & 6.290 & 1.00 & 0.00 \\
\hline-3.251 & -2.942 & 7.082 & 1.00 & 0.00 \\
\hline-2.640 & -2.798 & 7.969 & 1.00 & 0.00 \\
\hline-4.269 & -3.164 & 7.409 & 1.00 & 0.00 \\
\hline-3.230 & -1.620 & 6.306 & 1.00 & 0.00 \\
\hline-3.344 & -0.802 & 7.018 & 1.00 & 0.00 \\
\hline-2.022 & -1.461 & 5.558 & 1.00 & 0.00 \\
\hline-2.298 & -0.809 & 4.330 & 1.00 & 0.00 \\
\hline-1.638 & 0.048 & 4.184 & 1.00 & 0.00 \\
\hline-2.161 & -1.721 & 3.170 & 1.00 & 0.00 \\
\hline-2.201 & -3.092 & 3.076 & 1.00 & 0.00 \\
\hline-2.217 & -3.739 & 3.939 & 1.00 & 0.00 \\
\hline-2.390 & -3.529 & 1.857 & 1.00 & 0.00 \\
\hline-2.229 & -2.376 & 1.073 & 1.00 & 0.00 \\
\hline-2.258 & -2.138 & -0.350 & 1.00 & 0.00 \\
\hline-2.419 & -2.919 & -1.284 & 1.00 & 0.00 \\
\hline-2.122 & -0.815 & -0.690 & 1.00 & 0.00 \\
\hline-2.203 & -0.574 & -1.660 & 1.00 & 0.00 \\
\hline-1.849 & 0.147 & 0.201 & 1.00 & 0.00 \\
\hline-1.619 & 1.326 & -0.257 & 1.00 & 0.00 \\
\hline-1.401 & 2.012 & 0.442 & 1.00 & 0.00 \\
\hline-1.812 & 1.535 & -1.227 & 1.00 & 0.00 \\
\hline-1.792 & 0.005 & 1.508 & 1.00 & 0.00 \\
\hline-2.010 & -1.286 & 1.885 & 1.00 & 0.00 \\
\hline-4.321 & -1.489 & 5.259 & 1.00 & 0.00 \\
\hline-4.317 & -2.397 & 4.662 & 1.00 & 0.00 \\
\hline-3.751 & -0.343 & 4.413 & 1.00 & 0.00 \\
\hline-4.226 & -0.312 & 3.431 & 1.00 & 0.00 \\
\hline-3.772 & 0.945 & 5.025 & 1.00 & 0.00 \\
\hline-3.058 & 0.988 & 5.713 & 1.00 & 0.00 \\
\hline-5.600 & -1.259 & 5.806 & 1.00 & 0.00 \\
\hline-6.896 & -1.651 & 5.002 & 1.00 & 0.00 \\
\hline-8.042 & -1.383 & 5.883 & 1.00 & 0.00 \\
\hline-6.696 & -3.014 & 4.457 & 1.00 & 0.00 \\
\hline-6.929 & -0.607 & 3.796 & 1.00 & 0.00 \\
\hline-7.104 & 0.784 & 4.048 & 1.00 & 0.00 \\
\hline-6.359 & 1.123 & 4.765 & 1.00 & 0.00 \\
\hline-8.089 & 0.945 & 4.486 & 1.00 & 0.00 \\
\hline-6.991 & 1.672 & 2.804 & 1.00 & 0.00 \\
\hline-7.093 & 2.713 & 3.114 & 1.00 & 0.00 \\
\hline-5.749 & 1.519 & 2.114 & 1.00 & 0.00 \\
\hline-5.989 & 1.631 & 0.708 & 1.00 & 0.00 \\
\hline-5.369 & 2.421 & 0.283 & 1.00 & 0.00 \\
\hline & 0.361 & -0.018 & 1.00 & 0.00 \\
\hline-5.553 & -0.848 & 0.632 & 1.00 & 0.00 \\
\hline-5.590 & -0.886 & 1.714 & 1.00 & 0.00 \\
\hline & -1.996 & -0.080 & 1.00 & 0.00 \\
\hline-5 . & -2.946 & 0.420 & 1.00 & 0.00 \\
\hline-5.376 & -1.994 & -1.524 & 1.00 & 0.00 \\
\hline-5.303 & -2.972 & -2.251 & 1.00 & 0.00 \\
\hline & -0.756 & -2.085 & 1.00 & 0.00 \\
\hline & -0.736 & -3.088 & 1.00 & 0.00 \\
\hline & 0.418 & -1.417 & 1.00 & 0.00 \\
\hline & 1.451 & -2.067 & 1.00 & 0.00 \\
\hline-8 & 1.389 & 1.777 & 1.00 & 0.00 \\
\hline & 0.311 & 6 & 1.00 & 0.00 \\
\hline-7 & 2.028 & 0.531 & 1.00 & 0.00 \\
\hline-7 & 1.598 & -0.373 & 1.00 & 0.00 \\
\hline-7 . & 3.455 & 0.503 & 1.00 & 0.00 \\
\hline & 3.715 & 0.402 & 1.00 & 0.00 \\
\hline & 1.917 & 2.103 & 1.00 & 0.00 \\
\hline 10 & 1.363 & 1.604 & 1.00 & 0.00 \\
\hline
\end{tabular}

B-180 deg-MM.pdb

$\begin{array}{lrrll}\text { ATOM } & 1 & \text { H5T } & \text { RA5 } \\ \text { ATOM } & 2 & \text { O5' } & \text { RA5 } \\ \text { ATOM } & 3 & \text { C5' } & \text { RA5 } \\ \text { ATOM } & 4 & \text { H5' } 1 & \text { RA5 } \\ \text { ATOM } & 5 & \text { H5' } & \text { RA5 } \\ \text { ATOM } & 6 & \text { C4' } & \text { RA5 } \\ \text { ATOM } & 7 & \text { H } 4 & \text { RA5 } \\ \text { ATOM } & 8 & \text { O4' } & \text { RA5 } \\ \text { ATOM } & 9 & \text { C1' } & \text { RA5 } \\ \text { ATOM } & 10 & \text { H1' } & \text { RA5 } \\ \text { ATOM } & 11 & \text { N9 } & \text { RA5 } \\ \text { ATOM } & 12 & \text { C8 } & \text { RA5 } \\ \text { ATOM } & 13 & \text { H8 } & \text { RA5 } \\ \text { ATOM } & 14 & \text { N7 } & \text { RA5 } \\ \text { ATOM } & 15 & \text { C5 } & \text { RA5 } \\ \text { ATOM } & 16 & \text { C6 } & \text { RA5 } \\ \text { ATOM } & 17 & \text { N6 } & \text { RA5 } \\ \text { ATOM } & 18 & \text { H61 } & \text { RA5 } \\ \text { ATOM } & 19 & \text { H62 } & \text { RA5 } \\ \text { ATOM } & 20 & \text { N1 } & \text { RA5 }\end{array}$

$\begin{array}{lrrrr}-3.002 & -0.751 & -12.500 & 1.00 & 0.00 \\ -3.985 & -0.790 & -12.435 & 1.00 & 0.00 \\ -4.481 & 0.479 & -12.818 & 1.00 & 0.00 \\ -5.399 & 0.354 & -13.393 & 1.00 & 0.00 \\ -3.748 & 0.979 & -13.453 & 1.00 & 0.00 \\ -4.777 & 1.367 & -11.605 & 1.00 & 0.00 \\ -5.088 & 2.341 & -11.981 & 1.00 & 0.00 \\ -5.831 & 0.836 & -10.807 & 1.00 & 0.00 \\ -5.608 & 1.192 & -9.448 & 1.00 & 0.00 \\ -6.463 & 1.743 & -9.047 & 1.00 & 0.00 \\ -5.405 & -0.047 & -8.656 & 1.00 & 0.00 \\ -4.762 & -1.208 & -9.014 & 1.00 & 0.00 \\ -4.316 & -1.350 & -9.988 & 1.00 & 0.00 \\ -4.864 & -2.174 & -8.140 & 1.00 & 0.00 \\ -5.449 & -1.522 & -7.036 & 1.00 & 0.00 \\ -5.745 & -1.857 & -5.691 & 1.00 & 0.00 \\ -5.464 & -2.986 & -5.087 & 1.00 & 0.00 \\ -5.508 & -2.940 & -4.074 & 1.00 & 0.00 \\ -4.820 & -3.610 & -5.554 & 1.00 & 0.00 \\ -6.253 & -0.975 & -4.842 & 1.00 & 0.00\end{array}$




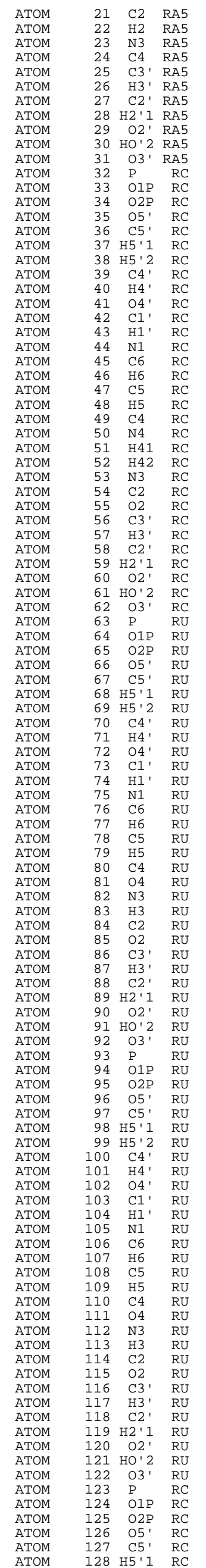

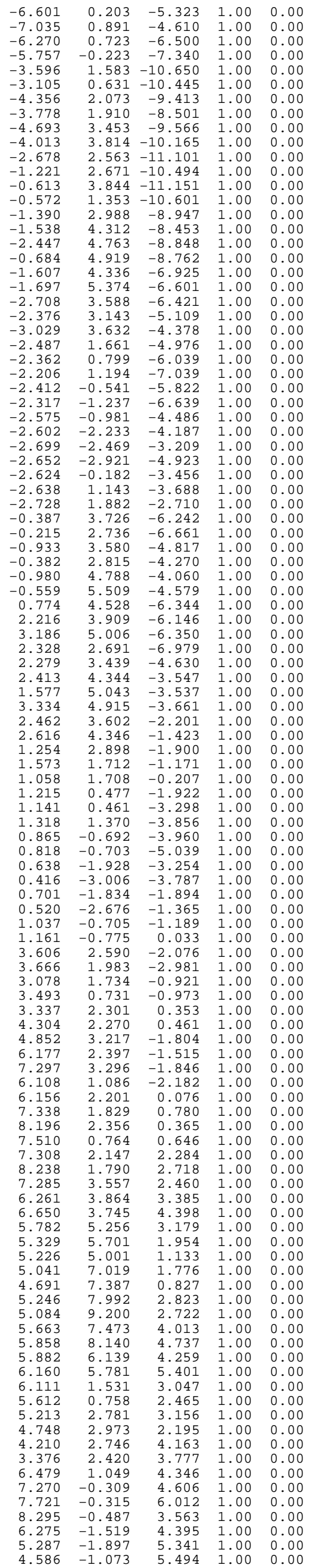




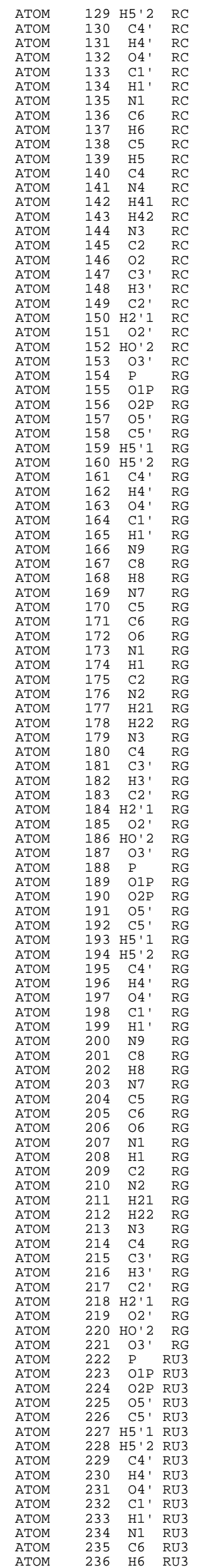

$\begin{array}{lllll}5.754 & -2.143 & 6.296 & 1.00 & 0.00\end{array}$

$\begin{array}{lllll}4.507 & -3.124 & 4.839 & 1.00 & 0.00\end{array}$

$\begin{array}{lllll}3.746 & -3.362 & 5.574 & 1.00 & 0.00 \\ 3.856 & -2.772 & 3.627 & 1.00 & 0.00\end{array}$

$\begin{array}{lllll}4.175 & -3.703 & 2.614 & 1.00 & 0.00\end{array}$

$\begin{array}{lllll}3.429 & -4.506 & 2.606 & 1.00 & 0.00\end{array}$

$\begin{array}{lllll}4.233 & -2.989 & 1.313 & 1.00 & 0.00\end{array}$

$\begin{array}{lllll}4.964 & -1.840 & 1.187 & 1.00 & 0.00\end{array}$

$\begin{array}{lllll}5.472 & -1.434 & 2.052 & 1.00 & 0.00\end{array}$

$\begin{array}{lllll}5.057 & -1.239 & -0.019 & 1.00 & 0.00\end{array}$

$\begin{array}{lllll}5.647 & -0.353 & -0.139 & 1.00 & 0.00\end{array}$

$\begin{array}{lllll}4.369 & -1.827 & -1.101 & 1.00 & 0.00\end{array}$

$\begin{array}{lllll}4.439 & -1.281 & -2.266 & 1.00 & 0.00\end{array}$

$\begin{array}{lllll}3.965 & -1.705 & -3.043 & 1.00 & 0.00\end{array}$

$\begin{array}{lllll}5.023 & -0.459 & -2.363 & 1.00 & 0.00\end{array}$

$\begin{array}{lllll}3.636 & -2.903 & -1.009 & 1.00 & 0.00\end{array}$

$\begin{array}{lllll}3.564 & -3.503 & 0.199 & 1.00 & 0.00\end{array}$

$\begin{array}{lllll}2.896 & -4.532 & 0.264 & 1.00 & 0.00 \\ 5.378 & -4.371 & 4.558 & 1.00 & 0.00\end{array}$

$\begin{array}{lllll}5.378 & -4.371 & 4.558 & 1.00 & 0.00 \\ 6.361 & -4.288 & 5.024 & 1.00 & 0.00\end{array}$

$\begin{array}{lllll}5.526 & -4.281 & 3.026 & 1.00 & 0.00\end{array}$

$\begin{array}{lllll}6.311 & -3.556 & 2.807 & 1.00 & 0.00\end{array}$

$\begin{array}{lllll}5.813 & -5.506 & 2.357 & 1.00 & 0.00\end{array}$

$\begin{array}{lllll}5.733 & -5.377 & 1.384 & 1.00 & 0.00\end{array}$

$\begin{array}{lllll}4.720 & -5.584 & 4.933 & 1.00 & 0.00\end{array}$

$\begin{array}{lllll}4.741 & -6.148 & 6.423 & 1.00 & 0.00\end{array}$

$\begin{array}{lllll}6.043 & -5.847 & 7.039 & 1.00 & 0.00\end{array}$

$\begin{array}{lllll}4.296 & -7.551 & 6.375 & 1.00 & 0.00 \\ 3.676 & -5.286 & 7.222 & 1.00 & 0.00\end{array}$

$\begin{array}{lllll}3.676 & -5.286 & 7.222 & 1.00 & 0.00 \\ 2.299 & -5.250 & 6.866 & 1.00 & 0.00\end{array}$

$\begin{array}{lllll}2.299 & -5.250 & 6.866 & 1.00 & 0.00 \\ 1.827 & -6.214 & 7.065 & 1.00 & 0.00\end{array}$

$\begin{array}{lllll}1.827 & -6.214 & 7.065 & 1.00 & 0.00 \\ 2.186 & -5.002 & 5.810 & 1.00 & 0.00\end{array}$

$\begin{array}{lllll}2.186 & -5.002 & 5.810 & 1.00 & 0.00 \\ 1.627 & -4.161 & 7.707 & 1.00 & 0.00\end{array}$

$\begin{array}{lllll}1.627 & -4.161 & 7.707 & 1.00 & 0.00 \\ 1.585 & -4.519 & 8.733 & 1.00 & 0.00\end{array}$

$\begin{array}{lllll}1.585 & -4.519 & 8.733 & 1.00 & 0.00 \\ 2.385 & -2.954 & 7.661 & 1.00 & 0.00\end{array}$

$\begin{array}{lllll}1.513 & -1.860 & 7.867 & 1.00 & 0.00 \\ 1.717 & -1.442 & 8.849 & 1.00 & 0.00\end{array}$

$\begin{array}{lllll}1.717 & -1.442 & 8.849 & 1.00 & 0.00 \\ 1.711 & -0.785 & 6.863 & 1.00 & 0.00\end{array}$

$\begin{array}{lllll}2.111 & 0.505 & 7.114 & 1.00 & 0.00\end{array}$

$\begin{array}{lllll}2.334 & 0.865 & 8.110 & 1.00 & 0.00\end{array}$

$\begin{array}{lllll}2.177 & 1.267 & 6.058 & 1.00 & 0.00\end{array}$

$\begin{array}{lllll}1.799 & 0.412 & 5.013 & 1.00 & 0.00\end{array}$

$\begin{array}{lllll}1.650 & 0.641 & 3.597 & 1.00 & 0.00\end{array}$

$\begin{array}{lllll}1.785 & 1.685 & 2.962 & 1.00 & 0.00\end{array}$

$\begin{array}{lllll}1.309 & -0.478 & 2.880 & 1.00 & 0.00 \\ 1.231 & -0.413 & 1.881 & 1.00 & 0.00\end{array}$

$\begin{array}{lllll}1.231 & -0.413 & 1.881 & 1.00 & 0.00 \\ 1.160 & -1.687 & 3.440 & 1.00 & 0.00\end{array}$

$\begin{array}{lllll}0.895 & -2.652 & 2.623 & 1.00 & 0.00\end{array}$

$\begin{array}{lllll}0.895 & -2.652 & 2.623 & 1.00 & 0.00 \\ 0.736 & -3.554 & 3.058 & 1.00 & 0.00\end{array}$

$\begin{array}{lllll}0.780 & -2.493 & 1.636 & 1.00 & 0.00\end{array}$

$\begin{array}{lllll}1.175 & -1.938 & 4.743 & 1.00 & 0.00 \\ 1.552 & -0.857 & 5.491 & 1.00 & 0.00\end{array}$

$\begin{array}{lllll}0.200 & -3.803 & 7.285 & 1.00 & 0.00\end{array}$

$\begin{array}{lllll}0.153 & -3.743 & 6.197 & 1.00 & 0.00\end{array}$

$\begin{array}{lllll}0.082 & -2.389 & 7.891 & 1.00 & 0.00\end{array}$

$\begin{array}{lllll}-0.547 & -1.764 & 7.269 & 1.00 & 0.00\end{array}$

$\begin{array}{lllll}-0.374 & -2.326 & 9.245 & 1.00 & 0.00\end{array}$

$\begin{array}{lllll}0.148 & -2.954 & 9.792 & 1.00 & 0.00\end{array}$

$\begin{array}{lllll}-0.774 & -4.702 & 7.799 & 1.00 & 0.00\end{array}$

$\begin{array}{lllll}-1.971 & -5.236 & 6.902 & 1.00 & 0.00 \\ -2.924 & -5.919 & 7.799 & 1.00 & 0.00\end{array}$

$\begin{array}{lllll}-2.924 & -5.919 & 7.799 & 1.00 & 0.00 \\ -1.385 & -5.991 & 5.777 & 1.00 & 0.00\end{array}$

$\begin{array}{lllll}-1.385 & -5.991 & 5.777 & 1.00 & 0.00 \\ -2.729 & -3.988 & 6.273 & 1.00 & 0.00\end{array}$

$\begin{array}{lllll}-2.729 & -3.988 & 6.273 & 1.00 & 0.00 \\ -3.240 & -2.933 & 7.074 & 1.00 & 0.00\end{array}$

$\begin{array}{lllll}-2.627 & -2.794 & 7.962 & 1.00 & 0.00\end{array}$

$\begin{array}{lllll}-4.257 & -3.159 & 7.401 & 1.00 & 0.00 \\ -3.220 & -1.607 & 6.306 & 1.00 & 0.00\end{array}$

$\begin{array}{lllll}-3.220 & -1.607 & 6.306 & 1.00 & 0.00 \\ -3.339 & -0.793 & 7.022 & 1.00 & 0.00\end{array}$

$\begin{array}{lllll}-2.011 & -1.439 & 5.562 & 1.00 & 0.00\end{array}$

$\begin{array}{lllll}-2.289 & -0.788 & 4.333 & 1.00 & 0.00\end{array}$

$\begin{array}{lllll}-1.630 & 0.071 & 4.186 & 1.00 & 0.00\end{array}$

$\begin{array}{lllll}-2.151 & -1.699 & 3.172 & 1.00 & 0.00\end{array}$

$\begin{array}{lllll}-2.174 & -3.070 & 3.077 & 1.00 & 0.00 \\ -2.176 & -3.717 & 3.939 & 1.00 & 0.00\end{array}$

$\begin{array}{llllll}-2.384 & -3.506 & 1.861 & 1.00 & 0.00\end{array}$

$\begin{array}{lllll}-2.384 & -3.506 & 1.861 & 1.00 & 0.00 \\ -2.231 & -2.353 & 1.076 & 1.00 & 0.00\end{array}$

$\begin{array}{llrrr}-2.231 & -2.353 & 1.076 & 1.00 & 0.00 \\ -2.271 & -2.114 & -0.347 & 1.00 & 0.00\end{array}$

$\begin{array}{llllll}-2.440 & -2.894 & -1.281 & 1.00 & 0.00\end{array}$

$\begin{array}{lllll}-2.137 & -0.791 & -0.687 & 1.00 & 0.00\end{array}$

$\begin{array}{rrrrr}-2.215 & -0.551 & -1.658 & 1.00 & 0.00 \\ -1.842 & 0.168 & 0.199 & 1.00 & 0.00\end{array}$

$\begin{array}{rrrrr}-1.842 & 0.168 & 0.199 & 1.00 & 0.00 \\ -1.582 & 1.339 & -0.265 & 1.00 & 0.00\end{array}$

$\begin{array}{lllll}-1.323 & 2.019 & 0.428 & 1.00 & 0.00\end{array}$

$\begin{array}{lllll}-1.764 & 1.548 & -1.236 & 1.00 & 0.00\end{array}$

$\begin{array}{lllll}-1.791 & 0.028 & 1.507 & 1.00 & 0.00\end{array}$

$\begin{array}{lllll}-2.006 & -1.263 & 1.886 & 1.00 & 0.00\end{array}$

$\begin{array}{lllll}-4.311 & -1.476 & 5.259 & 1.00 & 0.00 \\ -4.306 & -2.382 & 4.659 & 1.00 & 0.00\end{array}$

$\begin{array}{lllll}-3.743 & -0.327 & 4.417 & 1.00 & 0.00\end{array}$

$\begin{array}{lllll}-4.218 & -0.293 & 3.435 & 1.00 & 0.00\end{array}$

$\begin{array}{rrrrr}-4.218 & -0.293 & 3.435 & 1.00 & 0.00 \\ -3.771 & 0.958 & 5.036 & 1.00 & 0.00\end{array}$

$\begin{array}{lllll}-3.055 & 1.002 & 5.722 & 1.00 & 0.00\end{array}$

$\begin{array}{lrrrr}-3.055 & 1.002 & 5.722 & 1.00 & 0.00 \\ -5.591 & -1.251 & 5.807 & 1.00 & 0.00\end{array}$

$\begin{array}{lllll}-5.591 & -1.251 & 5.807 & 1.00 & 0.00 \\ -6.888 & -1.642 & 5.005 & 1.00 & 0.00\end{array}$

$\begin{array}{lllll}-8.032 & -1.373 & 5.886 & 1.00 & 0.00\end{array}$

$\begin{array}{lllll}-6.689 & -3.006 & 4.461 & 1.00 & 0.00 \\ -6.921 & -0.600 & 3.798 & 1.00 & 0.00\end{array}$

$\begin{array}{rrrrr}-6.921 & -0.600 & 3.798 & 1.00 & 0.00 \\ -7.095 & 0.792 & 4.050 & 1.00 & 0.00\end{array}$

$\begin{array}{lllll}-6.350 & 1.130 & 4.766 & 1.00 & 0.00\end{array}$

$\begin{array}{lllll}-8.080 & 0.954 & 4.489 & 1.00 & 0.00\end{array}$

$\begin{array}{lllll}-6.982 & 1.680 & 2.806 & 1.00 & 0.00\end{array}$

$\begin{array}{lllll}-7.081 & 2.721 & 3.115 & 1.00 & 0.00\end{array}$

$\begin{array}{lllll}-5.743 & 1.522 & 2.112 & 1.00 & 0.00\end{array}$

$\begin{array}{lllll}-5.985 & 1.635 & 0.707 & 1.00 & 0.00\end{array}$

$\begin{array}{lllll}-5.364 & 2.424 & 0.281 & 1.00 & 0.00\end{array}$

$\begin{array}{lllll}-5.702 & 0.364 & -0.020 & 1.00 & 0.00\end{array}$

$\begin{array}{lllll}-5.554 & -0.845 & 0.630 & 1.00 & 0.00 \\ -5.585 & -0.882 & 1.712 & 1.00 & 0.00\end{array}$ 


$\begin{array}{llll}\text { ATOM } & 237 & \text { C5 } & \text { RU3 } \\ \text { ATOM } & 238 & \text { H5 } & \text { RU3 } \\ \text { ATOM } & 239 & \text { C4 } & \text { RU3 } \\ \text { ATOM } & 240 & \text { O4 } & \text { RU3 } \\ \text { ATOM } & 241 & \text { N3 } & \text { RU3 } \\ \text { ATOM } & 242 & \text { H3 } & \text { RU3 } \\ \text { ATOM } & 243 & \text { C2 } & \text { RU3 } \\ \text { ATOM } & 244 & \text { O2 } & \text { RU3 } \\ \text { ATOM } & 245 & \text { C3' } & \text { RU3 } \\ \text { ATOM } & 246 & \text { H3' } & \text { RU3 } \\ \text { ATOM } & 247 & \text { C2' } & \text { RU3 } \\ \text { ATOM } & 248 & \text { H2'1 } & \text { RU3 } \\ \text { ATOM } & 249 & \text { O2' } & \text { RU3 } \\ \text { ATOM } & 250 & \text { HO'2 } & \text { RU3 } \\ \text { ATOM } & 251 & \text { O3' } & \text { RU3 } \\ \text { ATOM } & 252 & \text { H3T } & \text { RU3 }\end{array}$

$\begin{array}{rrrrr}-5.403 & -1.993 & -0.083 & 1.00 & 0.00 \\ -5.311 & -2.943 & 0.416 & 1.00 & 0.00 \\ -5.393 & -1.991 & -1.527 & 1.00 & 0.00 \\ -5.327 & -2.970 & -2.254 & 1.00 & 0.00 \\ -5.457 & -0.754 & -2.088 & 1.00 & 0.00 \\ -5.518 & -0.734 & -3.091 & 1.00 & 0.00 \\ -5.660 & 0.421 & -1.419 & 1.00 & 0.00 \\ -5.816 & 1.455 & -2.069 & 1.00 & 0.00 \\ -8.074 & 1.399 & 1.782 & 1.00 & 0.00 \\ -8.146 & 0.321 & 1.631 & 1.00 & 0.00 \\ -7.457 & 2.036 & 0.534 & 1.00 & 0.00 \\ -7.897 & 1.606 & -0.369 & 1.00 & 0.00 \\ -7.525 & 3.463 & 0.504 & 1.00 & 0.00 \\ -8.470 & 3.724 & 0.407 & 1.00 & 0.00 \\ -9.353 & 1.930 & 2.111 & 1.00 & 0.00 \\ -9.997 & 1.377 & 1.614 & 1.00 & 0.00\end{array}$

B-80deg-QM.pdb

ATOM 1 H5T RA5

ATOM 2 O5' RA5

ATOM 3 C5 1 RA5

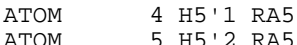

ATOM 6 C4 RA5

ATOM 7 H4' RA5

ATOM 8 O4' RA5

$\begin{array}{llll}8 & 9 & \text { C1' } & \text { RA5 } \\ & 10 & \text { H } 1 \text { ' } & \text { RA5 } 5\end{array}$

$\begin{array}{llll}\text { ATOM } & 10 & \text { H1 } & \text { RA5 } \\ \text { ATOM } & 11 & \text { N9 } & \text { RA5 }\end{array}$

$\begin{array}{llll}\text { ATOM } & 11 & \text { N9 } & \text { RA5 } \\ \text { ATOM } & 12 & \text { C8 } & \text { RA5 }\end{array}$

$\begin{array}{llll}\text { ATOM } & 12 & \text { C8 } & \text { RA5 } \\ \text { ATOM } & 13 & \text { H8 } & \text { RA5 }\end{array}$

ATOM 14 N7 RA5

ATOM 15 C5 RA5

$\begin{array}{llll}\text { ATOM } & 16 & \text { C6 } & \text { RA5 } \\ \text { ATOM } & 17 & \text { N6 } & \text { RA5 }\end{array}$

$\begin{array}{llll}\text { ATOM } & 17 & \text { N6 } & \text { RA5 } \\ \text { ATOM } & 18 & \text { H61 } & \text { RA5 }\end{array}$

$\begin{array}{llll}\text { ATOM } & 19 & \text { H61 } & \text { RA5 } \\ & 20 & \text { RA5 }\end{array}$

ATOM 20 N1 RA5

ATOM $21 \quad$ C2 RA5

$\begin{array}{llll}\text { ATOM } & 22 & \text { H2 } & \text { RA5 } \\ \text { ATOM } & 23 & \text { N3 } & \text { RA5 }\end{array}$

ATOM 24 C4 RA5

ATOM 25 C3. RA5

ATOM 26 H3' RA5

$\begin{array}{llll}\text { ATOM } & 26 & \text { H3' } & \text { RA5 } \\ \text { ATOM } & 27 & \text { C2 } & \text { RA5 }\end{array}$

$\begin{array}{llll}\text { ATOM } & 27 & \text { C2' RA5 } \\ \text { ATOM } & 28 & \text { H2' } & \text { RA5 }\end{array}$

$\begin{array}{llll}\text { ATOM } & 28 & \text { H2' } & \text { RA5 } \\ \text { ATOM } & 29 & 2 & \end{array}$

ATOM 30 HO'2 RA5

ATOM 31 O3' RA5

ATOM $\quad 32 \quad \mathrm{P} \quad \mathrm{RC}$

ATOM 33 O1P RC

$\begin{array}{llll}\text { ATOM } & 34 & \text { O2P } & \text { RC } \\ \text { ATOM } & 35 & 051 & \text { RC }\end{array}$

$\begin{array}{llll}\text { ATOM } & 35 & \text { O5 }^{\prime} & \text { RC } \\ \text { ATOM } & 36 & \text { C }^{\prime} & \text { RC }\end{array}$

ATOM $\quad 37$ H5' 1 RC

ATOM $38 \mathrm{H} 5 \mathrm{\prime}^{2} \mathrm{RC}$

ATOM $\quad 39$ C4' RC

ATOM 40 H4' RC

$\begin{array}{llll}\text { ATOM } & 41 & 04 & \text { RC }\end{array}$

$\begin{array}{llll}\text { ATOM } & 42 & \text { C1' } & \text { RC } \\ \text { ATOM } & 43 & \text { H1 } & \text { RC }\end{array}$

ATOM 44 N1 RC

$\begin{array}{llll}\text { ATOM } & 45 & \text { C6 } & \text { RC } \\ \text { ATOM } & 46 & \text { H6 } & \text { RC }\end{array}$

$\begin{array}{llll}\text { ATOM } & 46 & \text { H6 } & \text { RC } \\ \text { ATOM } & 47 & \text { C5 } & \text { RC }\end{array}$

$\begin{array}{llll}\text { ATOM } & 47 & \text { C5 } & \text { RC } \\ \text { ATOM } & 48 & \text { H5 } & \text { RC }\end{array}$

ATOM 49 C4 RC

$\begin{array}{llll}\text { ATOM } & 50 & \text { N4 } & \text { RC } \\ \text { ATOM } & 51 & \text { H41 } & \text { RC }\end{array}$

$\begin{array}{llll}\text { ATOM } & 51 & \text { H41 } & \text { RC } \\ \text { ATOM } & 52 & \text { H42 } & \text { RC }\end{array}$

ATOM 53 N3 RC

ATOM 54 C2 RC

$\begin{array}{llll}\text { ATOM } & 55 & \mathrm{O} 2 & \mathrm{RC}\end{array}$

$\begin{array}{llll}\text { ATOM } & 56 & \mathrm{C}^{\prime} & \mathrm{RC} \\ \text { ATOM } & 57 & \mathrm{H}^{\prime} & \mathrm{RC}\end{array}$

$\begin{array}{llll}\text { ATOM } & 57 & \text { H3' } & \text { RC } \\ \text { ATOM } & 58 & \text { C2 } & \text { RC }\end{array}$

ATOM 59 H2' 1 RC

$\begin{array}{llll}\text { ATOM } & 60 & \mathrm{O} 2^{\prime} & \mathrm{RC}\end{array}$

$\begin{array}{cccc}\text { ATOM } & 61 & \mathrm{HO}^{\prime} 2 & \mathrm{RC} \\ \text { ATOM } & 62 & \mathrm{O}^{\prime} & \mathrm{RC}\end{array}$

ATOM

ATOM

ATOM

ATOM

ATOM

ATOM

ATOM

ATOM

ATOM

ATOM

ATOM

ATOM

ATOM
ATOM

ATOM

ATOM
ATOM

ATOM
ATOM

ATOM

ATOM

ATOM

ATOM

$\begin{array}{lll}62 & \mathrm{O} 3 & \mathrm{RC} \\ 63 & \mathrm{P} & \mathrm{RU}\end{array}$

$\begin{array}{lll}63 & \mathrm{P} & \mathrm{RU} \\ 64 & \mathrm{O} 1 \mathrm{P} & \mathrm{RU}\end{array}$

65 O2P $\mathrm{RU}$

$665^{\prime} \quad \mathrm{RU}$

$\begin{array}{lll}67 & \mathrm{C} 5 & \mathrm{RU} \\ 68 & 5^{\prime} 1 & \mathrm{RU}\end{array}$

69 H5'2 RU

70 C4' RU

71 H4' $\mathrm{RU}$

$\begin{array}{lll}72 & 04 & \\ 73 & \mathrm{C} 1 & \mathrm{RU}\end{array}$

74 H1' RU

$\begin{array}{lll}75 & \mathrm{~N} 1 & \mathrm{RU} \\ 76 & \mathrm{C} 6 & \mathrm{RU}\end{array}$

77 H6 RU

$\begin{array}{lll}78 & \mathrm{C} 5 & \mathrm{RU} \\ 79 & \mathrm{H} 5 & \mathrm{RU}\end{array}$

80 C4 RU

$\begin{array}{lll}81 & 04 & R U\end{array}$

$\begin{array}{lllll}3.149 & -0.507 & 12.368 & 1.00 & 0.00\end{array}$ $\begin{array}{lllll}4.129 & -0.521 & 12.345 & 1.00 & 0.00\end{array}$ $\begin{array}{lllll}4.604 & 0.752 & 12.704 & 1.00 & 0.00\end{array}$ $\begin{array}{lllll}5.531 & 0.625 & 13.268 & 1.00 & 0.00\end{array}$ $\begin{array}{lllll}3.913 & 1.283 & 13.364 & 1.00 & 0.00\end{array}$

$\begin{array}{lllll}4.886 & 1.636 & 11.512 & 1.00 & 0.00\end{array}$

$\begin{array}{lllll}5.235 & 2.595 & 11.912 & 1.00 & 0.00\end{array}$

$\begin{array}{lllll}5.890 & 1.044 & 10.689 & 1.00 & 0.00\end{array}$

$\begin{array}{lllll}5.719 & 1.413 & 9.354 & 1.00 & 0.00\end{array}$

$\begin{array}{lllll}6.611 & 1.911 & 8.962 & 1.00 & 0.00\end{array}$

$\begin{array}{lllll}5.521 & 0.214 & 8.540 & 1.00 & 0.00\end{array}$

$\begin{array}{lllll}4.972 & -0.986 & 8.903 & 1.00 & 0.00 \\ 4.562 & -1.143 & 9.888 & 1.00 & 0.00\end{array}$

$\begin{array}{lllll}4.562 & -1.143 & 9.888 & 1.00 & 0.00 \\ 5.012 & -1.880 & 7.957 & 1.00 & 0.00\end{array}$

$\begin{array}{lllll}5.012 & -1.880 & 7.957 & 1.00 & 0.00 \\ 5.598 & -1.235 & 6.894 & 1.00 & 0.00\end{array}$

$\begin{array}{lllll}5.862 & -1.597 & 5.559 & 1.00 & 0.00\end{array}$

$\begin{array}{lllll}5.615 & -2.814 & 5.040 & 1.00 & 0.00\end{array}$

$\begin{array}{lllll}5.555 & -2.837 & 4.020 & 1.00 & 0.00\end{array}$

$\begin{array}{lllll}4.932 & -3.380 & 5.533 & 1.00 & 0.00\end{array}$

$\begin{array}{lllll}6.375 & -0.659 & 4.754 & 1.00 & 0.00\end{array}$

$\begin{array}{lllll}6.684 & 0.538 & 5.236 & 1.00 & 0.00\end{array}$

$\begin{array}{lllll}7.137 & 1.235 & 4.544 & 1.00 & 0.00\end{array}$

$\begin{array}{lllll}6.481 & 0.983 & 6.461 & 1.00 & 0.00\end{array}$

$\begin{array}{lllll}5.928 & 0.065 & 7.245 & 1.00 & 0.00\end{array}$

$\begin{array}{lllll}3.730 & 1.854 & 10.538 & 1.00 & 0.00\end{array}$

$\begin{array}{lllll}3.214 & 0.907 & 10.343 & 1.00 & 0.00\end{array}$

$\begin{array}{lllll}4.486 & 2.322 & 9.298 & 1.00 & 0.00\end{array}$

$\begin{array}{lllll}3.944 & 2.202 & 8.360 & 1.00 & 0.00\end{array}$

$\begin{array}{lllll}4.853 & 3.680 & 9.470 & 1.00 & 0.00\end{array}$

$\begin{array}{lllll}4.217 & 4.072 & 10.086 & 1.00 & 0.00 \\ 2.839 & 2.835 & 11.000 & 1.00 & 0.00\end{array}$

$\begin{array}{lllll}2.839 & 2.835 & 11.000 & 1.00 & 0.00 \\ 1.322 & 2.917 & 10.393 & 1.00 & 0.00\end{array}$

$\begin{array}{lllll}1.322 & 2.917 & 10.393 & 1.00 & 0.00 \\ 0.756 & 4.129 & 11.072 & 1.00 & 0.00\end{array}$

$\begin{array}{lllll}0.698 & 1.559 & 10.426 & 1.00 & 0.00\end{array}$

$\begin{array}{lllll}1.541 & 3.230 & 8.820 & 1.00 & 0.00\end{array}$

$\begin{array}{lllll}1.680 & 4.552 & 8.343 & 1.00 & 0.00\end{array}$

$\begin{array}{lllll}2.595 & 5.020 & 8.717 & 1.00 & 0.00\end{array}$

$\begin{array}{lllll}0.841 & 5.180 & 8.653 & 1.00 & 0.00\end{array}$

$\begin{array}{lllll}1.719 & 4.557 & 6.841 & 1.00 & 0.00\end{array}$

$\begin{array}{lllll}1.849 & 5.614 & 6.559 & 1.00 & 0.00\end{array}$

$\begin{array}{lllll}2.790 & 3.773 & 6.339 & 1.00 & 0.00\end{array}$

$\begin{array}{lllll}3.232 & 3.826 & 4.339 & 1.00 & 0.00\end{array}$

$\begin{array}{lllll}3.232 & 3.826 & 4.339 & 1.00 & 0.00 \\ 2.617 & 1.919 & 4.894 & 1.00 & 0.00\end{array}$

$\begin{array}{lllll}2.617 & 1.919 & 4.894 & 1.00 & 0.00 \\ 2.496 & 1.055 & 5.931 & 1.00 & 0.00\end{array}$

$\begin{array}{lllll}2.496 & 1.055 & 5.931 & 1.00 & 0.00 \\ 2.344 & 1.485 & 6.912 & 1.00 & 0.00\end{array}$

$\begin{array}{rrrrr}2.344 & 1.485 & 6.912 & 1.00 & 0.00 \\ 2.545 & -0.281 & 5.736 & 1.00 & 0.00\end{array}$

$\begin{array}{lllll}2.444 & -0.958 & 6.570 & 1.00 & 0.00\end{array}$

$\begin{array}{lllll}2.679 & -0.748 & 4.390 & 1.00 & 0.00\end{array}$

$\begin{array}{lllll}2.721 & -2.039 & 4.105 & 1.00 & 0.00\end{array}$

$\begin{array}{lllll}2.793 & -2.354 & 3.139 & 1.00 & 0.00\end{array}$

$\begin{array}{lllll}2.748 & -2.733 & 4.841 & 1.00 & 0.00\end{array}$

$\begin{array}{lllll}2.736 & 0.114 & 3.380 & 1.00 & 0.00\end{array}$

$\begin{array}{lllll}2.719 & 1.433 & 3.595 & 1.00 & 0.00 \\ 2.750 & 2.256 & 2.676 & 1.00 & 0.00\end{array}$

$\begin{array}{lllll}0.514 & 3.966 & 6.118 & 1.00 & 0.00\end{array}$

$\begin{array}{lllll}0.320 & 2.962 & 6.513 & 1.00 & 0.00\end{array}$

$\begin{array}{lllll}0.326 & 2.962 & 6.513 & 1.00 & 0.00 \\ 1.069 & 3.846 & 4.699 & 1.00 & 0.00\end{array}$

$\begin{array}{lllll}1.069 & 3.846 & 4.699 & 1.00 & 0.00\end{array}$

$\begin{array}{lllll}. .551 & 3.096 & 4.103 & 1.00 & 0.00 \\ 1.068 & 5.048 & 3.988 & 1.00 & 0.00\end{array}$

$\begin{array}{lllll}1.068 & 5.048 & 3.988 & 1.00 & 0.00 \\ 0.735 & 5.809 & 4.509 & 1.00 & 0.00\end{array}$

$\begin{array}{rrrrr}0.735 & 5.809 & 4.509 & 1.00 & 0.00 \\ -0.616 & 4.780 & 6.242 & 1.00 & 0.00\end{array}$

$\begin{array}{lllll}-0.616 & 4.780 & 6.242 & 1.00 & 0.00 \\ -2.105 & 4.148 & 6.037 & 1.00 & 0.00\end{array}$

$\begin{array}{lllll}-3.025 & 5.310 & 6.263 & 1.00 & 0.00\end{array}$

$\begin{array}{lllll}-2.237 & 2.867 & 6.808 & 1.00 & 0.00\end{array}$

$\begin{array}{lllll}-2.120 & 3.694 & 4.481 & 1.00 & 0.00\end{array}$

$\begin{array}{lllll}-2.275 & 4.609 & 3.419 & 1.00 & 0.00\end{array}$

$\begin{array}{lllll}-1.463 & 5.340 & 3.404 & 1.00 & 0.00 \\ -3.213 & 5.161 & 3.506 & 1.00 & 0.00\end{array}$

$\begin{array}{lllll}-2.286 & 3.883 & 2.093 & 1.00 & 0.00\end{array}$

$\begin{array}{lllll}-2.308 & 4.666 & 1.326 & 1.00 & 0.00\end{array}$

$\begin{array}{lllll}-1.103 & 3.100 & 1.937 & 1.00 & 0.00\end{array}$

$\begin{array}{lllll}-1.343 & 1.986 & 1.135 & 1.00 & 0.00\end{array}$

$\begin{array}{lllll}-0.665 & 1.984 & 0.282 & 1.00 & 0.00\end{array}$

$\begin{array}{lllll}-1.059 & 0.749 & 1.888 & 1.00 & 0.00 \\ -1.013 & 0.716 & 3.248 & 1.00 & 0.00\end{array}$

$\begin{array}{lllll}-1.203 & 1.651 & 3.753 & 1.00 & 0.00\end{array}$

$\begin{array}{lllll}-0.762 & -0.416 & 3.925 & 1.00 & 0.00\end{array}$

$\begin{array}{lllll}-0.744 & -0.413 & 5.004 & 1.00 & 0.00\end{array}$

$\begin{array}{lllll}-0.529 & -1.638 & 3.205 & 1.00 & 0.00\end{array}$

$\begin{array}{lllll}-0.312 & -2.724 & 3.727 & 1.00 & 0.00\end{array}$

$\begin{array}{lllll}-0.582 & -1.523 & 1.835 & 1.00 & 0.00\end{array}$

$\begin{array}{lllll}-0.443 & -2.368 & 1.265 & 1.00 & 0.00\end{array}$

$\begin{array}{rrrrr}-0.897 & -0.395 & 1.141 & 1.00 & 0.00 \\ -1.037 & -0.397 & -0.073 & 1.00 & 0.00\end{array}$

$\begin{array}{llllll}-3.408 & 2.876 & 1.828 & 1.00 & 0.00\end{array}$

$\begin{array}{lllll}-3.549 & 2.242 & 2.711 & 1.00 & 0.00\end{array}$

$\begin{array}{lllll}-2.849 & 2.242 & 2.711 & 1.00 & 0.00\end{array}$

$\begin{array}{lllll}-3.260 & 1.064 & 0.616 & 1.00 & 0.00\end{array}$ 


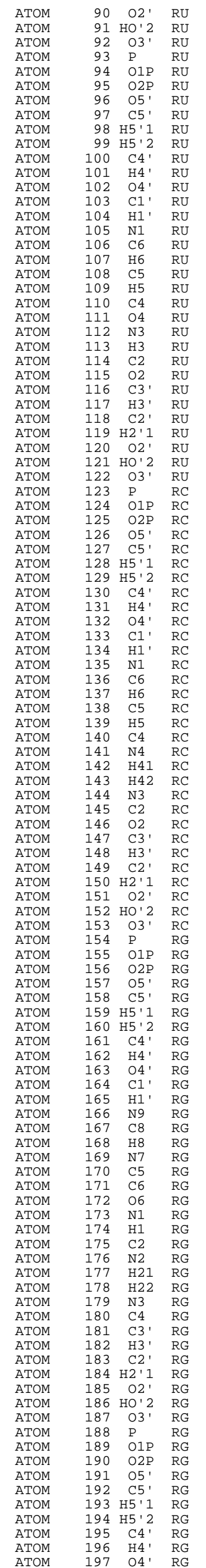

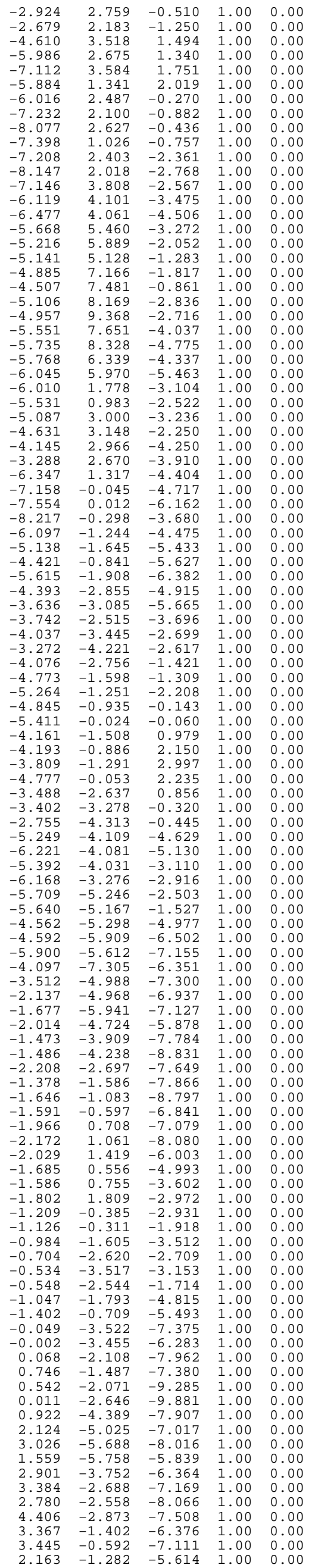




$\begin{array}{llll}\text { ATOM } & 198 & \text { C1' } & \text { RG } \\ \text { ATOM } & 199 & \text { H1' } & \text { RG } \\ \text { ATOM } & 200 & \text { N9 } & \text { RG } \\ \text { ATOM } & 201 & \text { C8 } & \text { RG } \\ \text { ATOM } & 202 & \text { H8 } & \text { RG } \\ \text { ATOM } & 203 & \text { N7 } & \text { RG } \\ \text { ATOM } & 204 & \text { C5 } & \text { RG } \\ \text { ATOM } & 205 & \text { C6 } & \text { RG } \\ \text { ATOM } & 206 & \text { O6 } & \text { RG } \\ \text { ATOM } & 207 & \text { N1' } & \text { RG } \\ \text { ATOM } & 208 & \text { H1 } & \text { RG } \\ \text { ATOM } & 209 & \text { C2 } & \text { RG } \\ \text { ATOM } & 210 & \text { N2 } & \text { RG } \\ \text { ATOM } & 211 & \text { H21 } & \text { RG } \\ \text { ATOM } & 212 & \text { H22 } & \text { RG } \\ \text { ATOM } & 213 & \text { N3 } & \text { RG } \\ \text { ATOM } & 214 & \text { C4' } & \text { RG } \\ \text { ATOM } & 215 & \text { C3' } & \text { RG } \\ \text { ATOM } & 216 & \text { H3' } & \text { RG } \\ \text { ATOM } & 217 & \text { C2' } & \text { RG } \\ \text { ATOM } & 218 & \text { H2'1 } & \text { RG } \\ \text { ATOM } & 219 & \text { O2' } & \text { RG } \\ \text { ATOM } & 220 & \text { HO'2 } & \text { RG } \\ \text { ATOM } & 221 & \text { O3' } & \text { RG } \\ \text { ATOM } & 222 & \text { P } & \text { RU3 } \\ \text { ATOM } & 223 & \text { O1P } & \text { RU3 } \\ \text { ATOM } & 224 & \text { O2P } & \text { RU3 } \\ \text { ATOM } & 225 & \text { O5' } & \text { RU3 } \\ \text { ATOM } & 226 & \text { C5' } & \text { RU3 } \\ \text { ATOM } & 227 & \text { H5' } 1 & \text { RU3 } \\ \text { ATOM } & 228 & \text { H5'2 } & \text { RU3 } \\ \text { ATOM } & 229 & \text { C4' } & \text { RU3 } \\ \text { ATOM } & 230 & \text { H4' } & \text { RU3 } \\ \text { ATOM } & 231 & \text { O4' } & \text { RU3 } \\ \text { ATOM } & 232 & \text { C1' } & \text { RU3 } \\ \text { ATOM } & 233 & \text { H1' } & \text { RU3 } \\ \text { ATOM } & 234 & \text { N1 } & \text { RU3 } \\ \text { ATOM } & 235 & \text { C6 } & \text { RU3 } \\ \text { ATOM } & 236 & \text { H6 } & \text { RU3 } \\ \text { ATOM } & 237 & \text { C5 } & \text { RU3 } \\ \text { ATOM } & 238 & \text { H5 } & \text { RU3 } \\ \text { ATOM } & 239 & \text { C4 } & \text { RU3 } \\ \text { ATOM } & 240 & \text { O4 } & \text { RU3 } \\ \text { ATOM } & 241 & \text { N3 } & \text { RU3 } \\ \text { ATOM } & 242 & \text { H3 } & \text { RU3 } \\ \text { ATOM } & 243 & \text { C2 } & \text { RU3 } \\ \text { ATOM } & 244 & \text { O2 } & \text { RU3 } \\ \text { ATOM } & 245 & \text { C3' } & \text { RU3 } \\ \text { ATOM } & 246 & \text { H3' } & \text { RU3 } \\ \text { ATOM } & 247 & \text { C2' } & \text { RU3 } \\ \text { ATOM } & 248 & \text { H2''1 } & \text { RU3 } \\ \text { ATOM } & 249 & \text { O2' } & \text { RU3 } \\ \text { ATOM } & 250 & \text { HO'2 } & \text { RU3 } \\ \text { ATOM } & 251 & \text { O3' } & \text { RU3 } \\ \text { ATOM } & 252 & \text { H3T } & \text { RU3 } \\ \text { END } & & & \\ \text { ATD } & & & \end{array}$

$\begin{array}{llllll}2.399 & -0.611 & -4.410 & 1.00 & 0.00\end{array}$

$\begin{array}{lllll}1.685 & 0.207 & -4.273 & 1.00 & 0.00\end{array}$

$\begin{array}{lllll}2.248 & -1.502 & -3.272 & 1.00 & 0.00 \\ 2.419 & -2.869 & -3.171 & 1.00 & 0.00\end{array}$

$\begin{array}{lllll}2.435 & -3.514 & -4.036 & 1.00 & 0.00\end{array}$

$\begin{array}{lllll}2.457 & -3.277 & -1.941 & 1.00 & 0.00\end{array}$

$\begin{array}{lllll}2.326 & -2.142 & -1.187 & 1.00 & 0.00\end{array}$

$\begin{array}{lllll}2.405 & -1.926 & 0.216 & 1.00 & 0.00 \\ 2.601 & -2.748 & 1.107 & 1.00 & 0.00\end{array}$

$\begin{array}{lllll}2.252 & -0.587 & 0.547 & 1.00 & 0.00\end{array}$

$\begin{array}{lllll}2.252 & -0.387 & 0.547 & 1.00 & 0.00\end{array}$

$\begin{array}{lllll}2.001 & 0.418 & -0.342 & 1.00 & 0.00\end{array}$

$\begin{array}{lllll}1.707 & 1.618 & 0.176 & 1.00 & 0.00\end{array}$

$\begin{array}{rrrrr}1.707 & 1.618 & 0.176 & 1.00 & 0.00 \\ 1.541 & 2.343 & -0.501 & 1.00 & 0.00\end{array}$

$\begin{array}{rrrrr}1.541 & 2.343 & -0.501 & 1.00 & 0.00 \\ 2.019 & 1.881 & 1.112 & 1.00 & 0.00\end{array}$

$\begin{array}{rrrrr}2.019 & 1.881 & 1.112 & 1.00 & 0.00 \\ 1.991 & 0.243 & -1.640 & 1.00 & 0.00\end{array}$

$\begin{array}{lllll}1.991 & 0.243 & -1.640 & 1.00 & 0.00 \\ 2.175 & -1.033 & -1.997 & 1.00 & 0.00\end{array}$

$\begin{array}{lllll}2.175 & -1.033 & -1.997 & 1.00 & 0.00 \\ 4.451 & -1.216 & -5.330 & 1.00 & 0.00\end{array}$

$\begin{array}{lllll}4.500 & -2.109 & -4.696 & 1.00 & 0.00\end{array}$

$\begin{array}{lllll}3.841 & -0.093 & -4.496 & 1.00 & 0.00\end{array}$

$\begin{array}{lllll}4.305 & 0.017 & -3.516 & 1.00 & 0.00 \\ 3.902 & 1.168 & -5.119 & 1.00 & 0.00\end{array}$

$\begin{array}{lllll}3.181 & 1.285 & -5.798 & 1.00 & 0.00\end{array}$

$\begin{array}{lllll}5.689 & -0.950 & -5.904 & 1.00 & 0.00\end{array}$

$\begin{array}{lllll}7.041 & -1.400 & -5.109 & 1.00 & 0.00\end{array}$

$\begin{array}{lllll}8.151 & -1.104 & -6.055 & 1.00 & 0.00\end{array}$

$\begin{array}{lllll}6.835 & -2.772 & -4.525 & 1.00 & 0.00\end{array}$

$\begin{array}{lllll}7.063 & -0.369 & -3.841 & 1.00 & 0.00\end{array}$

$\begin{array}{lllll}7.238 & 1.012 & -4.120 & 1.00 & 0.00\end{array}$

$\begin{array}{lllll}7.488 & 1.367 & -4.828 & 1.00 & 0.00\end{array}$

$\begin{array}{lllll}6.488 & 1.367 & -4.828 & 1.00 & 0.00 \\ 8.219 & 1.200 & -4.561 & 1.00 & 0.00\end{array}$

$\begin{array}{lllll}8.219 & 1.200 & -4.561 & 1.00 & 0.00 \\ 7.167 & 1.863 & -2.878 & 1.00 & 0.00\end{array}$

$\begin{array}{lllll}7.167 & 1.863 & -2.878 & 1.00 & 0.00 \\ 7.226 & 2.902 & -3.228 & 1.00 & 0.00\end{array}$

$\begin{array}{lllll}7.226 & 2.902 & -3.228 & 1.00 & 0.00 \\ 5.948 & 1.680 & -2.157 & 1.00 & 0.00\end{array}$

$\begin{array}{lllll}6.151 & 1.854 & -0.786 & 1.00 & 0.00\end{array}$

$\begin{array}{lllll}5.471 & 2.610 & -0.392 & 1.00 & 0.00\end{array}$

$\begin{array}{lllll}5.851 & 0.596 & -0.070 & 1.00 & 0.00\end{array}$

$\begin{array}{lllll}5.710 & -0.605 & -0.704 & 1.00 & 0.00\end{array}$

$\begin{array}{lllll}5.774 & -0.583 & -1.781 & 1.00 & 0.00\end{array}$

$\begin{array}{lllll}5.518 & -1.754 & -0.036 & 1.00 & 0.00\end{array}$

$\begin{array}{lllll}5.423 & -2.684 & -0.570 & 1.00 & 0.00\end{array}$

$\begin{array}{lllll}5.517 & -1.749 & 1.405 & 1.00 & 0.00\end{array}$

$\begin{array}{lllll}5.453 & -2.732 & 2.127 & 1.00 & 0.00\end{array}$

$\begin{array}{lllll}5.616 & -0.492 & 1.961 & 1.00 & 0.00\end{array}$

$\begin{array}{lllll}5.757 & -0.466 & 2.981 & 1.00 & 0.00\end{array}$

$\begin{array}{rrrrr}5.757 & -0.466 & 2.981 & 1.00 & 0.00 \\ 5.802 & 0.678 & 1.304 & 1.00 & 0.00\end{array}$

$\begin{array}{lllll}5.802 & 0.678 & 1.304 & 1.00 & 0.00 \\ 5.926 & 1.741 & 1.895 & 1.00 & 0.00\end{array}$

$\begin{array}{rrrrr}5.926 & 1.741 & 1.895 & 1.00 & 0.00 \\ 8.239 & 1.612 & -1.833 & 1.00 & 0.00\end{array}$

$\begin{array}{lllll}8.239 & 1.612 & -1.833 & 1.00 & 0.00 \\ 8.307 & 0.529 & -1.667 & 1.00 & 0.00\end{array}$

$\begin{array}{lllll}7.617 & 2.261 & -0.597 & 1.00 & 0.00\end{array}$

$\begin{array}{lllll}8.045 & 1.868 & 0.330 & 1.00 & 0.00\end{array}$

$\begin{array}{lllll}7.695 & 3.659 & -0.638 & 1.00 & 0.00\end{array}$

$\begin{array}{lllll}8.627 & 3.939 & -0.502 & 1.00 & 0.00 \\ 9.486 & 2.134 & -2.195 & 1.00 & 0.00\end{array}$

$\begin{array}{rrrrr}9.486 & 2.134 & -2.195 & 1.00 & 0.00 \\ 10.157 & 1.577 & -1.745 & 1.00 & 0.00\end{array}$

B-180 deg-QM.pdb

ATOM 1 H5T RA5 $\begin{array}{lll}1 & \text { H5T } & \text { RA5 } \\ 2 & \text { O5 } & \text { RA5 }\end{array}$

$\begin{array}{llll}\text { ATOM } & 2 & \text { O5' } & \text { RA5 } \\ \text { ATOM } & 3 & \text { C5 } & \text { RA5 }\end{array}$

$\begin{array}{llll}\text { ATOM } & 3 & \text { C5 } & \text { RA5 } \\ \text { ATOM } & 4 & \text { H5 } 1 & \text { RA5 }\end{array}$

ATOM 5 H5'2 RA5

$\begin{array}{llll}\text { ATOM } & 6 & \text { C4 } & \text { RA5 } \\ \text { ATOM } & 7 & \text { H4 } & \text { RA5 }\end{array}$

$\begin{array}{llll}\text { ATOM } & 7 & \text { H4' } & \text { RA5 } \\ \text { ATOM } & 8 & \text { O4' } & \text { RA5 }\end{array}$

ATOM 9 C1' RA5

ATOM 10 H1' RA5

ATOM 11 N9 RA5

$\begin{array}{llll}\text { ATOM } & 12 & \text { C8 } & \text { RA5 } \\ & 13 & \text { H8 } & \text { RA5 }\end{array}$

$\begin{array}{llll}\text { ATOM } & 13 & \text { H8 } & \text { RA5 } \\ \text { ATOM } & 14 & \text { N7 } & \text { RA5 }\end{array}$

ATOM 15 C5 RA5

ATOM $16 \quad$ C6 RA5

ATOM 17 N6 RA5

ATOM $\quad 18$ H61 RA5

ATOM 19 H62 RA5

ATOM 20 N1 RA5

ATOM 21 C2 RA5

$\begin{array}{llll}\text { ATOM } & 22 & \text { H2 } & \text { RA5 } \\ \text { ATOM } & 23 & \text { N3 } & \text { RA5 }\end{array}$

ATOM 24 C4 RA5

ATOM 25 C3' RA5

ATOM 26 H3' RA5

ATOM 27 C2' RA5

ATOM 28 H2' 1 RA5

$\begin{array}{llll}\text { ATOM } & 29 & \text { O2' } & \text { RA5 } \\ \text { ATOM } & 30 & \text { HO' } 2 & \text { RA5 }\end{array}$

30 HO'2 RA5

31 O3' RA5

$\begin{array}{lll}32 & \mathrm{P} & \mathrm{RC} \\ 33 & \mathrm{O} 1 \mathrm{P} & \mathrm{RC} \\ 34 & \mathrm{O} 2 \mathrm{P} & \mathrm{RC}\end{array}$

$\begin{array}{lll}33 & 01 P & R C \\ 34 & 02 P & R C\end{array}$

$\begin{array}{lll}34 & \text { O2P } & \text { RC } \\ 35 & \text { O5 } & \text { RC }\end{array}$

$\begin{array}{lll}35 & 05^{\prime} & \text { RC } \\ 36 & \mathrm{C}^{\prime} & \mathrm{RC}\end{array}$

37 H5' 1 RC

38 H5'2 RC

$\begin{array}{lll}39 & \mathrm{C}^{\prime} & \mathrm{RC} \\ 40 & \mathrm{H}^{\prime} & \mathrm{RC}\end{array}$

41 O4' RC

42 C1' RC

$\begin{array}{lll}43 & \mathrm{H}^{\prime} & \mathrm{RC} \\ 44 & \mathrm{~N} 1 & \mathrm{RC}\end{array}$

$\begin{array}{lll}45 & \mathrm{C} 6 & \mathrm{RC} \\ 46 & \mathrm{H} 6 & \mathrm{RC}\end{array}$

$\begin{array}{lll}46 & \mathrm{H} 6 & \mathrm{RC} \\ 47 & \mathrm{C} 5 & \mathrm{RC}\end{array}$

$\begin{array}{lll}47 & \mathrm{C} 5 & \mathrm{RC} \\ 48 & \mathrm{H} 5 & \mathrm{RC}\end{array}$

$\begin{array}{lll}49 & \mathrm{C} 4 & \mathrm{RC} \\ 50 & \mathrm{~N} 4 & \mathrm{RC}\end{array}$

$\begin{array}{lllll}-3.164 & -0.513 & -12.366 & 1.00 & 0.00\end{array}$

$\begin{array}{lllll}-4.143 & -0.526 & -12.346 & 1.00 & 0.00\end{array}$

$\begin{array}{lllll}-4.617 & 0.747 & -12.706 & 1.00 & 0.00\end{array}$

$\begin{array}{lllll}-5.543 & 0.621 & -13.271 & 1.00 & 0.00\end{array}$

$\begin{array}{lllll}-3.923 & 1.278 & -13.365 & 1.00 & 0.00\end{array}$

$\begin{array}{lllll}-4.898 & 1.632 & -11.514 & 1.00 & 0.00\end{array}$

$\begin{array}{lllll}-5.245 & 2.591 & -11.915 & 1.00 & 0.00 \\ -5.904 & 1.043 & -10.692 & 1.00 & 0.00\end{array}$

$\begin{array}{lllll}-5.904 & 1.043 & -10.692 & 1.00 & 0.00 \\ -5.733 & 1.410 & -9.356 & 1.00 & 0.00\end{array}$

$\begin{array}{lllll}-5.733 & 1.410 & -9.356 & 1.00 & 0.00 \\ -6.625 & 1.911 & -8.965 & 1.00 & 0.00\end{array}$

$\begin{array}{lllll}-5.538 & 0.211 & -8.543 & 1.00 & 0.00\end{array}$

$\begin{array}{lllll}-4.990 & -0.990 & -8.906 & 1.00 & 0.00\end{array}$

$\begin{array}{lllll}-4.579 & -1.147 & -9.891 & 1.00 & 0.00\end{array}$

$\begin{array}{lllll}-5.033 & -1.885 & -7.960 & 1.00 & 0.00\end{array}$

$\begin{array}{lllll}-5.621 & -1.240 & -6.898 & 1.00 & 0.00\end{array}$

$\begin{array}{llll}-1.601 & -5.563 & 1.00 & 0.00\end{array}$

$\begin{array}{lllll}-5.640 & -2.819 & -5.044 & 1.00 & 0.00\end{array}$

$\begin{array}{lllll}-5.579 & -2.839 & -4.023 & 1.00 & 0.00\end{array}$

$\begin{array}{lllll}-4.957 & -3.385 & -5.535 & 1.00 & 0.00\end{array}$

$\begin{array}{lllll}-6.400 & -0.664 & -4.759 & 1.00 & 0.00\end{array}$

$\begin{array}{lllll}-6.705 & 0.534 & -5.240 & 1.00 & 0.00\end{array}$

$\begin{array}{lllll}-7.158 & 1.231 & -4.549 & 1.00 & 0.00\end{array}$

$\begin{array}{lllll}-6.499 & 0.979 & -6.465 & 1.00 & 0.00 \\ -5.946 & 0.061 & -7.249 & 1.00 & 0.00\end{array}$

$\begin{array}{lllll}-5.946 & 0.061 & -7.249 & 1.00 & 0.00 \\ -3.742 & 1.848 & -10.539 & 1.00 & 0.00\end{array}$

$\begin{array}{lllll}-3.229 & 0.900 & -10.345 & 1.00 & 0.00\end{array}$

$\begin{array}{lllll}-4.499 & 2.317 & -9.300 & 1.00 & 0.00\end{array}$

$\begin{array}{lllll}-3.958 & 2.195 & -8.361 & 1.00 & 0.00\end{array}$

$\begin{array}{lllll}-4.862 & 3.676 & -9.470 & 1.00 & 0.00\end{array}$

$\begin{array}{lllll}-4.226 & 4.067 & -10.088 & 1.00 & 0.00\end{array}$

$\begin{array}{lllll}-2.850 & 2.827 & -11.002 & 1.00 & 0.00\end{array}$

$\begin{array}{lllll}-1.334 & 2.909 & -10.390 & 1.00 & 0.00\end{array}$

$\begin{array}{lllll}-0.764 & 4.118 & -11.070 & 1.00 & 0.00\end{array}$

$\begin{array}{lllll}-0.712 & 1.549 & -10.421 & 1.00 & 0.00\end{array}$

$\begin{array}{lllll}-1.558 & 3.225 & -8.818 & 1.00 & 0.00 \\ -1.694 & 4.547 & -8.343 & 1.00 & 0.00\end{array}$

$\begin{array}{lllll}-1.694 & 4.547 & -8.343 & 1.00 & 0.00 \\ -2.609 & 5.016 & -8.717 & 1.00 & 0.00\end{array}$

$\begin{array}{llllll}-0.854 & 5.174 & -8.654 & 1.00 & 0.00\end{array}$

$\begin{array}{lllll}-1.732 & 4.553 & -6.841 & 1.00 & 0.00\end{array}$

$\begin{array}{lllll}-1.856 & 5.610 & -6.559 & 1.00 & 0.00\end{array}$

$\begin{array}{lllll}-2.806 & 3.774 & -6.338 & 1.00 & 0.00\end{array}$

$\begin{array}{lllll}-2.522 & 3.380 & -5.021 & 1.00 & 0.00\end{array}$

$\begin{array}{lllll}-3.242 & 3.827 & -4.336 & 1.00 & 0.00\end{array}$

$\begin{array}{lllll}-2.637 & 1.917 & -4.896 & 1.00 & 0.00\end{array}$

$\begin{array}{lllll}-2.520 & 1.053 & -5.933 & 1.00 & 0.00\end{array}$

$\begin{array}{lllll}-2.367 & 1.483 & -6.914 & 1.00 & 0.00\end{array}$

$\begin{array}{lllll}-2.574 & -0.281 & -5.737 & 1.00 & 0.00\end{array}$

$\begin{array}{lllll}-2.475 & -0.960 & -6.571 & 1.00 & 0.00\end{array}$

$\begin{array}{lllll}-2.708 & -0.749 & -4.390 & 1.00 & 0.00\end{array}$ 


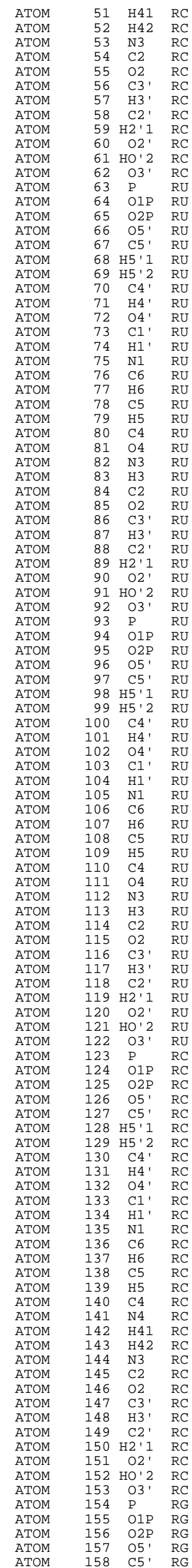

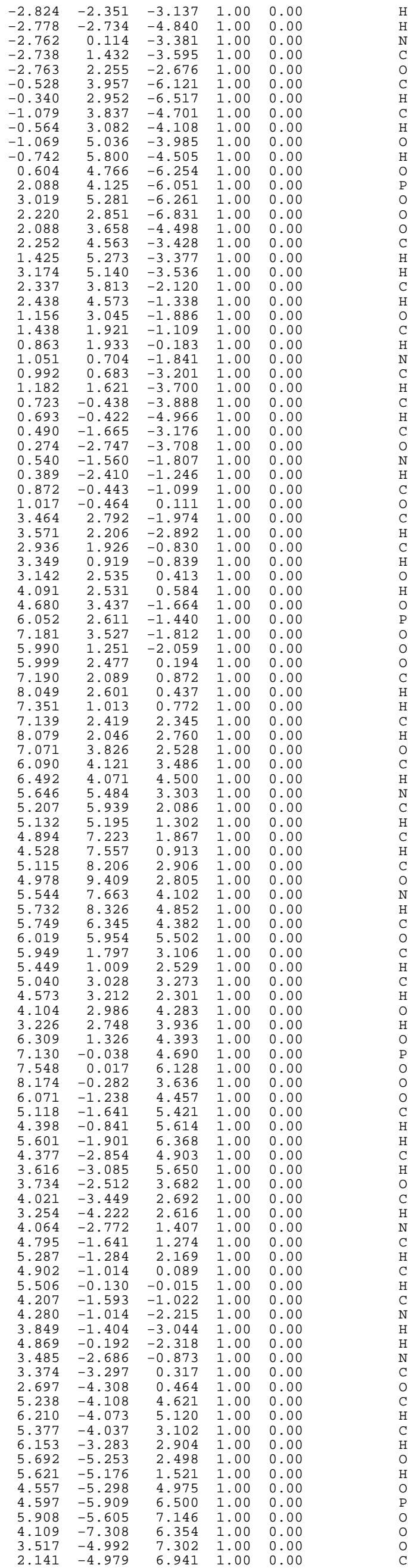




\begin{tabular}{|c|c|c|c|}
\hline ATOM & 159 & H5' 1 & RG \\
\hline ATOM & 160 & H5 ' 2 & RG \\
\hline ATOM & 161 & C4' & RG \\
\hline ATOM & 162 & $\mathrm{H}_{4}{ }^{\prime}$ & RG \\
\hline ATOM & 163 & $04{ }^{\prime}$ & RG \\
\hline ATOM & 164 & $\mathrm{C} 11^{\prime}$ & RG \\
\hline ATOM & 165 & H1 ' & RG \\
\hline ATOM & 166 & N9 & RG \\
\hline ATOM & 167 & C8 & RG \\
\hline ATOM & 168 & H8 & RG \\
\hline ATOM & 169 & N7 & RG \\
\hline ATOM & 170 & C5 & RG \\
\hline ATOM & 171 & C6 & RG \\
\hline ATOM & 172 & 06 & RG \\
\hline ATOM & 173 & N1 & RG \\
\hline ATOM & 174 & $\mathrm{H} 1$ & RG \\
\hline ATOM & 175 & $\mathrm{C} 2$ & RG \\
\hline ATOM & 176 & N2 & RG \\
\hline ATOM & 177 & H 21 & RG \\
\hline АTOM & 178 & H 22 & RG \\
\hline ATOM & 179 & N3 & RG \\
\hline ATOM & 180 & $\mathrm{C} 4$ & RG \\
\hline ATOM & 181 & C3' & RG \\
\hline ATOM & 182 & H3' & RG \\
\hline ATOM & 183 & C2 ${ }^{\prime}$ & RG \\
\hline ATOM & 184 & $\mathrm{H} 2{ }^{\prime} 1$ & RG \\
\hline ATOM & 185 & $02^{\prime}$ & RG \\
\hline ATOM & 186 & HO' 2 & RG \\
\hline ATOM & 187 & O3' & RG \\
\hline ATOM & 188 & P & RG \\
\hline ATOM & 189 & O1P & RG \\
\hline ATOM & 190 & $\mathrm{O} 2 \mathrm{P}$ & RG \\
\hline ATOM & 191 & $05^{\prime}$ & RG \\
\hline ATOM & 192 & C5' & RG \\
\hline ATOM & 193 & H5' 1 & RG \\
\hline АTOM & 194 & H5' 2 & RG \\
\hline ATOM & 195 & C4 & RG \\
\hline ATOM & 196 & H4 ' & RG \\
\hline ATOM & 197 & O4' & RG \\
\hline ATOM & 198 & C1' & RG \\
\hline ATOM & 199 & H1 ' & RG \\
\hline ATOM & 200 & N9 & RG \\
\hline ATOM & 201 & C8 & RG \\
\hline ATOM & 202 & H8 & RG \\
\hline ATOM & 203 & N7 & RG \\
\hline ATOM & 204 & C5 & RG \\
\hline ATOM & 205 & C6 & RG \\
\hline ATOM & 206 & 06 & RG \\
\hline ATOM & 207 & N1 & RG \\
\hline ATOM & 208 & H1 & RG \\
\hline ATOM & 209 & $\mathrm{C} 2$ & RG \\
\hline ATOM & 210 & N2 & RG \\
\hline ATOM & 211 & H 21 & RG \\
\hline ATOM & 212 & H 22 & RG \\
\hline ATOM & 213 & N3 & RG \\
\hline АTOM & 214 & C4 & RG \\
\hline ATOM & 215 & C3' & RG \\
\hline ATOM & 216 & H3' & RG \\
\hline ATOM & 217 & $\mathrm{C} 2{ }^{\prime}$ & RG \\
\hline ATOM & 218 & H2' 1 & RG \\
\hline ATOM & 219 & $02^{\prime}$ & RG \\
\hline АTOM & 220 & HO' 2 & RG \\
\hline ATOM & 221 & $03^{\prime}$ & RG \\
\hline ATOM & 222 & P & RU3 \\
\hline ATOM & 223 & O1P & RU3 \\
\hline ATOM & 224 & $\mathrm{O} 2 \mathrm{P}$ & RU3 \\
\hline ATOM & 225 & $05^{\prime}$ & RU3 \\
\hline ATOM & 226 & C5' & RU3 \\
\hline ATOM & 227 & H5' 1 & RU3 \\
\hline ATOM & 228 & H5' 2 & RU3 \\
\hline ATOM & 229 & C4' & RU3 \\
\hline ATOM & 230 & $\mathrm{H}_{4}{ }^{\prime}$ & RU3 \\
\hline ATOM & 231 & $04^{\prime}$ & RU3 \\
\hline ATOM & 232 & $\mathrm{C} 1{ }^{\prime}$ & RU3 \\
\hline ATOM & 233 & $\mathrm{H} 1^{\prime}$ & RU3 \\
\hline ATOM & 234 & N1 & RU3 \\
\hline ATOM & 235 & $\mathrm{C} 6$ & RU3 \\
\hline ATOM & 236 & $\mathrm{H} 6$ & RU3 \\
\hline ATOM & 237 & C5 & RU3 \\
\hline ATOM & 238 & H5 & RU3 \\
\hline ATOM & 239 & $\mathrm{C} 4$ & RU3 \\
\hline ATOM & 240 & 04 & RU3 \\
\hline ATOM & 241 & N3 & RU3 \\
\hline ATOM & 242 & H3 & RU3 \\
\hline ATOM & 243 & $\mathrm{C} 2$ & RU3 \\
\hline ATOM & 244 & 02 & RU3 \\
\hline ATOM & 245 & C3' & RU3 \\
\hline ATOM & 246 & H3 ' & RU3 \\
\hline ATOM & 247 & $\mathrm{C} 2{ }^{\prime}$ & RU3 \\
\hline ATOM & 248 & $\mathrm{H} 2{ }^{\prime} 1$ & RU3 \\
\hline ATOM & 249 & $02^{\prime}$ & RU3 \\
\hline ATOM & 250 & HO' 2 & RU3 \\
\hline ATOM & 251 & $03^{\prime}$ & RU3 \\
\hline ATOM & 252 & H3 T & RU3 \\
\hline
\end{tabular}

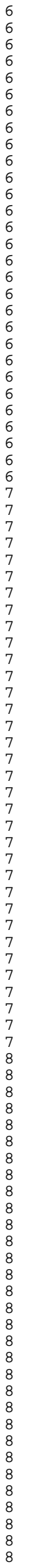

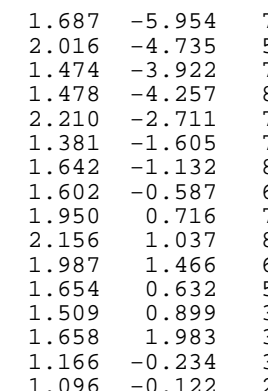

$\begin{array}{ll}1.166 & -0.234 \\ 1.096 & -0.122\end{array}$

$\begin{array}{ll}1.096 & -0.122 \\ 0.987 & -1.483\end{array}$

$\begin{array}{ll}0.735 & -2.478 \\ 0.575 & -3.395\end{array}$

$\begin{array}{ll}0.575 & -3.395 \\ 0.575 & -2.353\end{array}$

$\begin{array}{ll}0.575 & -2.353 \\ 1.063 & -1.721\end{array}$

$1.404-0.654$

$0.054-3.530$

$0.018-3.449$

$\begin{array}{ll}-0.068 & -2.124 \\ -0.737 & -1.496\end{array}$

$\begin{array}{ll}-0.737 & -1.496 \\ -0.562 & -2.102\end{array}$

$-0.028-2.673$

$-0.920-4.403$

$\begin{array}{ll}-0.920 & -4.403 \\ -2.122 & -5.019\end{array}$

$-3.030-5.691$

$\begin{array}{ll}-1.551 & -5.731 \\ -2.894 & -3.736\end{array}$

$\begin{array}{ll}-3.378 & -2.681\end{array}$

$-2.773-2.557$

$\begin{array}{ll}-4.400 & -2.870 \\ -3.364 & -1.390\end{array}$

$-3.445-0.584$

$\begin{array}{ll}-2.159 & -1.263 \\ -2.397 & -0.590\end{array}$

$\begin{array}{lr}-2.397 & -0.590 \\ -1.684 & 0.230\end{array}$

$-2.246-1.479$

$\begin{array}{ll}-2.408 & -2.847\end{array}$

$-2.411-3.492$

$-2.455-3.255$

$-2.335-2.119$

$-2.427-1.903$

$\begin{array}{ll}-2.639 & -2.722 \\ -2.267 & -0.565\end{array}$

$\begin{array}{ll}-2.267 & -0.565 \\ -2.402 & -0.340\end{array}$

$\begin{array}{lllll}-1.661 & 1.626 & -0.185 & 1.00 & 0.00\end{array}$

$\begin{array}{lllll}-1.461 & 2.345 & 0.491 & 1.00 & 0.00\end{array}$

$\begin{array}{lllll}-1.970 & 1.896 & -1.118 & 1.00 & 0.00\end{array}$

$\begin{array}{lllll}-1.991 & 0.265 & 1.636 & 1.00 & 0.00\end{array}$

$\begin{array}{lllll}-2.182 & -1.010 & 1.995 & 1.00 & 0.00\end{array}$

$\begin{array}{lllll}-4.448 & -1.203 & 5.328 & 1.00 & 0.00\end{array}$

$\begin{array}{lllll}-4.496 & -2.095 & 4.692 & 1.00 & 0.00\end{array}$

$\begin{array}{lllll}-3.840 & -0.076 & 4.499 & 1.00 & 0.00\end{array}$

$\begin{array}{lllll}-4.303 & 0.035 & 3.519 & 1.00 & 0.00 \\ -3.906 & 1.181 & 5.128 & 1.00 & 0.00\end{array}$

$\begin{array}{lllll}-3.906 & 1.181 & 5.128 & 1.00 & 0.00 \\ -3.185 & 1.298 & 5.807 & 1.00 & 0.00\end{array}$

$\begin{array}{rrrrr}-3.185 & 1.298 & 5.807 & 1.00 & 0.00 \\ -5.686 & -0.940 & 5.905 & 1.00 & 0.00\end{array}$

$\begin{array}{lllll}-7.039 & -1.387 & 5.112 & 1.00 & 0.00\end{array}$

$\begin{array}{lllll}-8.147 & -1.089 & 6.060 & 1.00 & 0.00\end{array}$

$\begin{array}{lllll}-6.837 & -2.760 & 4.529 & 1.00 & 0.00\end{array}$

$\begin{array}{lllll}-7.063 & -0.358 & 3.843 & 1.00 & 0.00\end{array}$

$\begin{array}{lllll}-7.235 & 1.023 & 4.121 & 1.00 & 0.00\end{array}$

$\begin{array}{lllll}-8.216 & 1.213 & 4.563 & 1.00 & 0.00\end{array}$

$\begin{array}{lllll}-7.164 & 1.873 & 2.878 & 1.00 & 0.00\end{array}$

$\begin{array}{lllll}-7.219 & 2.912 & 3.226 & 1.00 & 0.00 \\ -5.948 & 1.685 & 2.153 & 1.00 & 0.00\end{array}$

$\begin{array}{lllll}-5.473 & 2.611 & 0.387 & 1.00 & 0.00\end{array}$

$\begin{array}{lllll}-5.473 & 2.611 & 0.387 & 1.00 & 0.00 \\ -5.861 & 0.599 & 0.067 & 1.00 & 0.00\end{array}$

$\begin{array}{llllll}-5.861 & 0.599 & 0.067 & 1.00 & 0.00\end{array}$

$\begin{array}{lllll}-5.720 & -0.603 & 0.700 & 1.00 & 0.00\end{array}$

$\begin{array}{lllll}-5.537 & -1.753 & 0.032 & 1.00 & 0.00\end{array}$

$\begin{array}{lllll}-5.537 & -1.753 & 0.032 & 1.00 & 0.00 \\ -5.441 & -2.683 & 0.566 & 1.00 & 0.00\end{array}$

$\begin{array}{llrrr}-5.441 & -2.683 & 0.566 & 1.00 & 0.00 \\ -5.543 & -1.749 & -1.410 & 1.00 & 0.00\end{array}$

$\begin{array}{lllll}-5.487 & -2.732 & -2.131 & 1.00 & 0.00\end{array}$

$\begin{array}{lllll}-5.639 & -0.492 & -1.965 & 1.00 & 0.00\end{array}$

$\begin{array}{lllll}-5.781 & -0.466 & -2.984 & 1.00 & 0.00\end{array}$

$\begin{array}{lllll}-5.817 & 0.680 & -1.308 & 1.00 & 0.00\end{array}$

$\begin{array}{lllll}-5.940 & 1.742 & -1.900 & 1.00 & 0.00\end{array}$

$\begin{array}{lllll}-8.240 & 1.623 & 1.836 & 1.00 & 0.00 \\ -8.310 & 0.541 & 1.672 & 1.00 & 0.00\end{array}$

$\begin{array}{lllll}-7.619 & 2.270 & 0.598 & 1.00 & 0.00\end{array}$

$\begin{array}{lllll}-8.051 & 1.877 & -0.327 & 1.00 & 0.00\end{array}$

$\begin{array}{lllll}-7.694 & 3.668 & 0.636 & 1.00 & 0.00\end{array}$

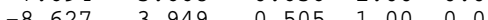

$\begin{array}{rrrrr}-9.485 & 2.149 & 2.201 & 1.00 & 0.00 \\ -10.158 & 1.593 & 1.753 & 1.00 & 0.00\end{array}$ $\begin{array}{lllll}-2.402 & -0.340 & -1.546 & 1.00 & 0.00\end{array}$

$\begin{array}{lllll}-6.483 & 1.378 & 4.828 & 1.00 & 0.00\end{array}$

$\begin{array}{lllll}-6.154 & 1.858 & 0.783 & 1.00 & 0.00\end{array}$

$\begin{array}{lllll}-8.627 & 3.949 & 0.5365 & 1.00 & 0.00\end{array}$

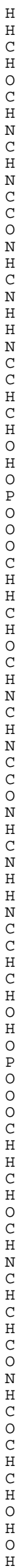

C-80deg-MM.pdb

\begin{tabular}{|c|c|c|c|}
\hline ATOM & 1 & H5T & RA5 \\
\hline ATOM & 2 & $05^{\prime}$ & RA5 \\
\hline ATOM & 3 & C5 ${ }^{\prime}$ & RA5 \\
\hline ATOM & 4 & H5' 1 & RA5 \\
\hline ATOM & 5 & H5' 2 & RA5 \\
\hline ATOM & 6 & C4' & RA5 \\
\hline ATOM & 7 & H4 ' & RA5 \\
\hline ATOM & 8 & O4' & RA5 \\
\hline ATOM & 9 & $\mathrm{C} 1^{\prime}$ & RA5 \\
\hline ATOM & 10 & H1 ' & RA5 \\
\hline ATOM & 1. & N9 & 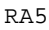 \\
\hline
\end{tabular}

$\begin{aligned}-7.703 & -9.980 \\ -7.725 & -9.200 \\ -8.954 & -9.155 \\ -9.777 & -9.367 \\ -8.954 & -9.911 \\ -9.192 & -7.777 \\ -10.155 & -7.799 \\ -9.216 & -6.755 \\ -8.593 & -5.582 \\ -9.249 & -4.716 \\ -7.332 & -5.298\end{aligned}$

$\begin{array}{lll}4.470 & 1.00 & 0.00\end{array}$

$\begin{array}{lll}3.882 & 1.00 & 0.00\end{array}$

$\begin{array}{lll}3.858 & 1.00 & 0.00\end{array}$

$2.388 \quad 1.00 \quad 0.00$

$2.544 \quad 1.00 \quad 0.00$

$3.0211 .00 \quad 0.00$

$\begin{array}{lll}3.131 & 1.00 & 0.00\end{array}$

$\begin{array}{lll}3.752 & 1.00 & 0.00\end{array}$
3.5413 .000 .00 


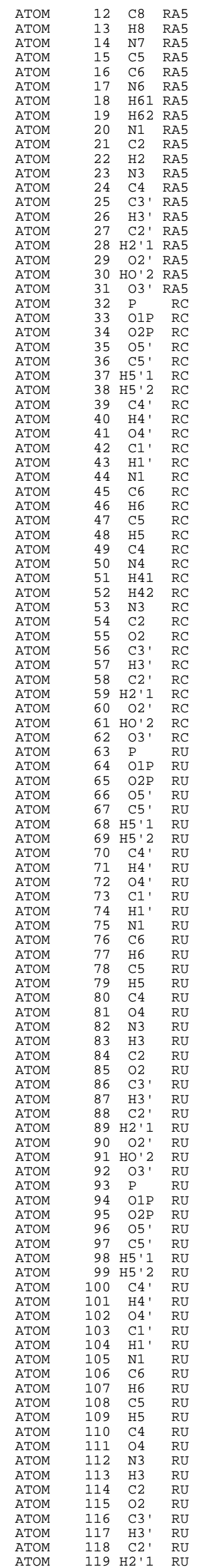

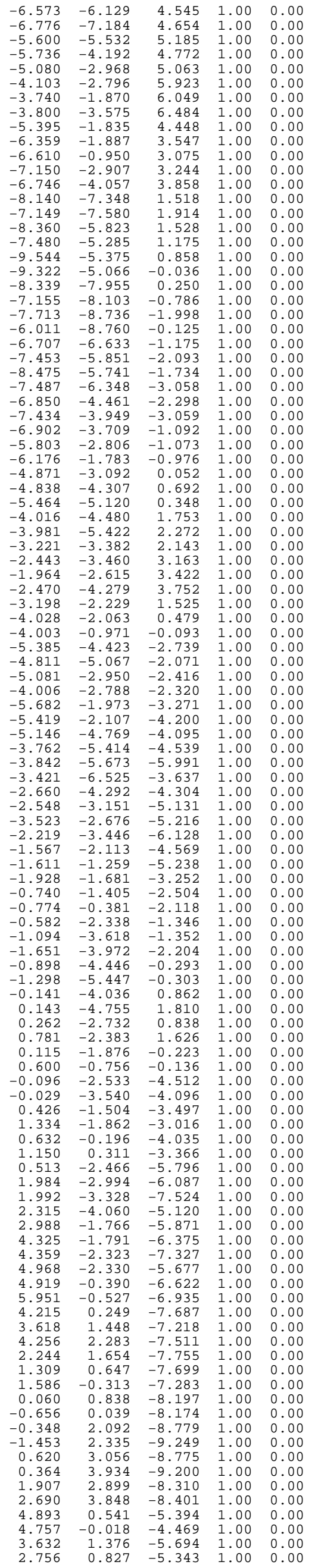




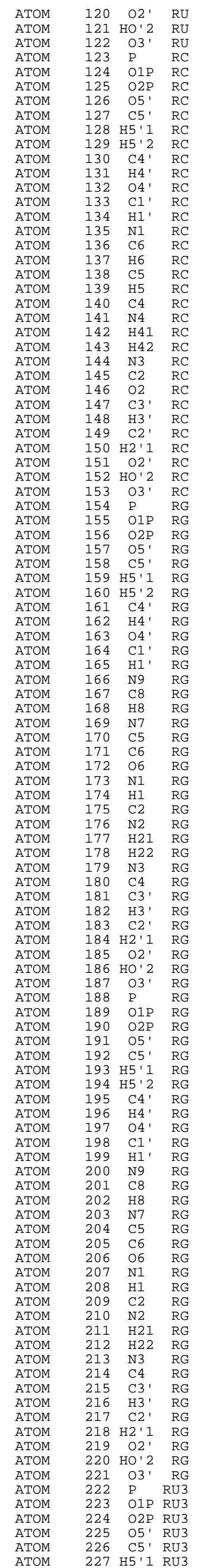

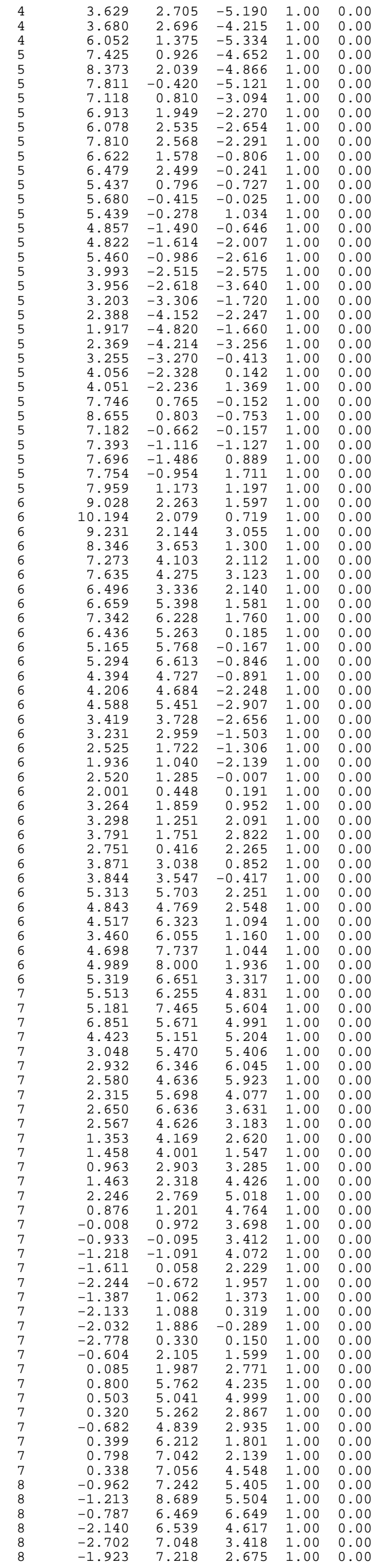




\begin{tabular}{|c|c|c|c|}
\hline ATOM & 228 & H5' 2 & RU3 \\
\hline ATOM & 229 & C4 ' & RU3 \\
\hline ATOM & 230 & $\mathrm{H} 44^{\prime}$ & RU3 \\
\hline ATOM & 231 & $04{ }^{\prime}$ & RU3 \\
\hline ATOM & 232 & $\mathrm{C} 1^{\prime}$ & RU3 \\
\hline ATOM & 233 & H1' & RU3 \\
\hline ATOM & 234 & N1 & RU3 \\
\hline ATOM & 235 & C6 & RU3 \\
\hline ATOM & 236 & H 6 & RU3 \\
\hline ATOM & 237 & C5 & RU3 \\
\hline ATOM & 238 & H5 & RU3 \\
\hline ATOM & 239 & C4 & RU3 \\
\hline ATOM & 240 & 04 & RU3 \\
\hline ATOM & 241 & N3 & RU3 \\
\hline ATOM & 242 & H3 & RU3 \\
\hline ATOM & 243 & $\mathrm{C} 2$ & RU3 \\
\hline ATOM & 244 & 02 & RU3 \\
\hline ATOM & 245 & C3' & RU3 \\
\hline ATOM & 246 & H3 & RU3 \\
\hline ATOM & 247 & $\mathrm{C} 2{ }^{\prime}$ & RU3 \\
\hline ATOM & 248 & $\mathrm{H} 2 \mathbf{2}^{\prime} 1$ & RU3 \\
\hline ATOM & 249 & $\mathrm{O} 2^{\prime}$ & RU3 \\
\hline ATOM & 250 & HO' 2 & RU3 \\
\hline ATOM & 251 & O3' & RU3 \\
\hline ATOM & 252 & H3T & RU3 \\
\hline
\end{tabular}

$\begin{array}{rrrrr}-3.216 & 7.990 & 3.614 & 1.00 & 0.00 \\ -3.709 & 6.031 & 2.856 & 1.00 & 0.00 \\ -4.108 & 6.404 & 1.913 & 1.00 & 0.00 \\ -3.094 & 4.762 & 2.632 & 1.00 & 0.00 \\ -4.048 & 3.731 & 2.858 & 1.00 & 0.00 \\ -4.222 & 3.209 & 1.913 & 1.00 & 0.00 \\ -3.606 & 2.738 & 3.882 & 1.00 & 0.00 \\ -2.735 & 3.040 & 4.911 & 1.00 & 0.00 \\ -2.279 & 4.023 & 4.947 & 1.00 & 0.00 \\ -2.469 & 2.116 & 5.881 & 1.00 & 0.00 \\ -1.796 & 2.349 & 6.693 & 1.00 & 0.00 \\ -3.078 & 0.803 & 5.875 & 1.00 & 0.00 \\ -2.949 & -0.091 & 6.705 & 1.00 & 0.00 \\ -3.884 & 0.575 & 4.803 & 1.00 & 0.00 \\ -4.326 & -0.329 & 4.747 & 1.00 & 0.00 \\ -4.220 & 1.484 & 3.843 & 1.00 & 0.00 \\ -5.086 & 1.170 & 3.032 & 1.00 & 0.00 \\ -4.890 & 5.759 & 3.791 & 1.00 & 0.00 \\ -4.529 & 5.644 & 4.814 & 1.00 & 0.00 \\ -5.365 & 4.396 & 3.280 & 1.00 & 0.00 \\ -5.862 & 3.851 & 4.085 & 1.00 & 0.00 \\ -6.203 & 4.467 & 2.129 & 1.00 & 0.00 \\ -6.994 & 5.004 & 2.365 & 1.00 & 0.00 \\ -5.911 & 6.747 & 3.747 & 1.00 & 0.00 \\ -6.589 & 6.496 & 4.426 & 1.00 & 0.00\end{array}$

C-180 deg-MM. pdb

ATOM 1 H5T RA5

ATOM 2 O5' RA5

$\begin{array}{llll}\text { ATOM } & 3 & \text { C5 } & \text { RA5 } \\ \text { ATOM } & 4 & \text { H5' } 1 & \text { RA5 }\end{array}$

ATOM 5 H5'2 RA5

ATOM 6 C4' RA5

$\begin{array}{llll}\text { ATOM } & 7 & \text { H4 ' } & \text { RA5 } \\ \text { ATOM } & 8 & \text { O4' } & \text { RA5 }\end{array}$

$\begin{array}{llll}\text { ATOM } & 8 & \text { O4' } & \text { RA5 } \\ \text { ATOM } & 9 & \text { C1', RA5 }\end{array}$

$\begin{array}{lrll}\text { ATOM } & 9 & \text { C1' } & \text { RA5 } \\ & 10 & \text { H1' } & \text { RA5 }\end{array}$

$\begin{array}{llll}\text { ATOM } & 10 & \text { H1' RA5 } \\ \text { ATOM } & 11 & \text { N9 } & \text { RA5 }\end{array}$

ATOM 12 C8 RA5

ATOM 13 H8 RA5

$\begin{array}{llll}\text { ATOM } & 14 & \text { N7 } & \text { RA5 }\end{array}$

$\begin{array}{llll}\text { ATOM } & 15 & \text { C5 } & \text { RA5 } \\ \text { ATOM } & 16 & \text { C6 } & \text { RA5 }\end{array}$

$\begin{array}{llll}\text { ATOM } & 16 & \text { C6 } & \text { RA5 } \\ \text { ATOM } & 17 & \text { N6 } & \text { RA5 }\end{array}$

$\begin{array}{llll}\text { ATOM } & 17 & \text { N6 } & \text { RA5 } \\ \text { ATOM } & 18 & \text { H61 } & \text { RA5 }\end{array}$

$\begin{array}{llll}\text { ATOM } & 18 & \text { H61 } & \text { RA5 } \\ \text { ATOM } & 19 & \text { H62 } & \text { RA5 }\end{array}$

$\begin{array}{llll}\text { ATOM } & 19 & \text { H62 } & \text { RA5 } \\ \text { ATOM } & 20 & \text { N1 } & \text { RA5 }\end{array}$

ATOM 21 C2 RA5

ATOM 22 H2 RA5

$\begin{array}{llll}\text { ATOM } & 23 & \text { N3 } & \text { RA5 } \\ \text { ATOM } & 24 & \text { C4 } & \text { RA5 }\end{array}$

ATOM 25 C3' RA5

ATOM 26 H3' RA5

ATOM 27 C2' RA5

ATOM 28 H2'1 RA5

ATOM 29 O2' RA5

ATOM 30 HO'2 RA5

ATOM 31 O3' RA5

$\begin{array}{llll}\text { ATOM } & 32 & \mathrm{P} & \mathrm{RC}\end{array}$

$\begin{array}{llll}\text { ATOM } & 33 & 01 \mathrm{P} & \mathrm{RC}\end{array}$

$\begin{array}{llll}\text { ATOM } & 34 & \text { O2P } & \text { RC } \\ \text { ATOM } & 35 & \text { O5 }^{\prime} & \text { RC }\end{array}$

ATOM 36 C5' RC

ATOM 37 H5'1 RC

ATOM 38 H5'2 RC

$\begin{array}{llll}\text { ATOM } & 39 & \text { C }^{\prime}{ }^{\prime} & \text { RC } \\ \text { ATOM } & 40 & \text { H }^{\prime} & \text { RC }\end{array}$

ATOM 41 O4' RC

$\begin{array}{llll}\text { ATOM } & 42 & \mathrm{C}^{\prime} & \mathrm{RC} \\ \text { ATOM } & 43 & \mathrm{H}^{\prime} & \mathrm{RC}\end{array}$

ATOM 44 N1 $\mathrm{RC}$

ATOM 45 C6 RC

ATOM 46 H6 RC

ATOM 47 C5 $\mathrm{RC}$

ATOM 48 H5 RC

ATOM 49 C4 RC

$\begin{array}{llll}\text { ATOM } & 50 & \text { N4 } & \text { RC } \\ \text { ATOM } & 51 & \text { H41 } & \text { RC }\end{array}$

$\begin{array}{llll}\text { ATOM } & 52 & \mathrm{H} 42 & \mathrm{RC}\end{array}$

ATOM 53 N3 RC

$\begin{array}{llll}\text { ATOM } & 54 & \text { C2 } & \text { RC } \\ \text { ATOM } & 55 & \text { O2 } & \text { RC }\end{array}$

ATOM $\quad 56 \mathrm{C}^{\prime}$ RC

$\begin{array}{llll}\text { ATOM } & 57 & \text { H3' } & \text { RC } \\ \text { ATOM } & 58 & C^{\prime} & \text { RC }\end{array}$

ATOM $\quad 59$ H2' 1 RC

ATOM 60 O2' RC

ATOM $61 \mathrm{HO}^{\prime 2} \mathrm{RC}$

ATOM $62 \mathrm{O}^{\prime} \mathrm{RC}$

ATOM $63 \mathrm{P} \quad \mathrm{RU}$

$\begin{array}{llll}\text { ATOM } & 64 & 01 \mathrm{P} & \mathrm{RU}\end{array}$

$\begin{array}{llll}\text { ATOM } & 65 & \mathrm{O} 2 \mathrm{P} & \mathrm{RU}\end{array}$

ATOM 66 O5' RU

$\begin{array}{llll}\text { ATOM } & 67 & \text { C5 }^{\prime} & \text { RU } \\ \text { ATOM } & 68 & 5^{\prime} 1 & \text { RU }\end{array}$

$\begin{array}{llll}\text { ATOM } & 68 & \text { H5 }^{\prime} 1 & \text { RU } \\ \text { ATOM } & 69 & \text { H5 }^{\prime 2} & \text { RU }\end{array}$

$\begin{array}{llll}\text { ATOM } & 69 & \text { H5 }^{\prime} 2 & \text { RU } \\ \text { ATOM } & 70 & \text { C4' } & \text { RU }\end{array}$

ATOM 71 H4' RU

$\begin{array}{llll}\text { ATOM } & 72 & \mathrm{O}^{\prime} & \mathrm{RU}\end{array}$

$\begin{array}{llll}\text { ATOM } & 73 & \mathrm{C} 1 & \text { RU } \\ \text { ATOM } & 74 & \mathrm{H}^{\prime} & \mathrm{RU}\end{array}$

$\begin{array}{llll}\text { ATOM } & 75 & \text { N1 } & \text { RU }\end{array}$

$\begin{array}{llll}\text { ATOM } & 76 & \mathrm{C} 6 & \mathrm{RU}\end{array}$

$\begin{array}{llll}\text { ATOM } & 77 & \text { H6 } & \text { RU } \\ \text { ATOM } & 78 & \text { C5 } & \text { RU }\end{array}$

$\begin{array}{llll}\text { ATOM } & 78 & \text { C5 } & \text { RU } \\ \text { ATOM } & 79 & \text { H5 } & \text { RU }\end{array}$

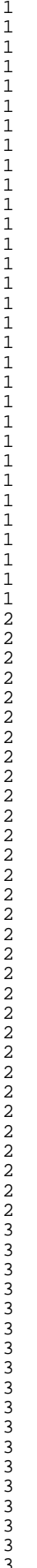

\begin{tabular}{|c|c|c|c|c|}
\hline 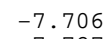 & -9.941 & 4.551 & 1.00 & .00 \\
\hline 27 & -9.16 & 3.957 & 1.00 & 0.00 \\
\hline-8.95 & -9.128 & 246 & 1.00 & 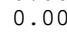 \\
\hline & -9.33 & 94 & 00 & 0 \\
\hline & (1) & & 00 & 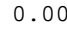 \\
\hline & & & & \\
\hline 10 & & & 0 & \\
\hline & -6.723 & & .00 & .00 \\
\hline & & & & \\
\hline & -4 & & .00 & .00 \\
\hline & -5 & & & \\
\hline-6.5 & 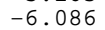 & & 0 & \\
\hline & & & & \\
\hline & -5 . & & 0 & \\
\hline & & & & \\
\hline & & & & \\
\hline & & & & \\
\hline & -1 & & & \\
\hline & -3 & & 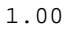 & \\
\hline & & & & \\
\hline & -1 & & 0 & \\
\hline & & & & \\
\hline & -2 & & & \\
\hline & -4 & & & \\
\hline & -7 & & & \\
\hline & -7 & & & \\
\hline & -5 & 1 . & & \\
\hline & & & & \\
\hline & & ( & & \\
\hline & -5 & 0 & & \\
\hline & -7 & 0 & & \\
\hline & -8 & 0 & & \\
\hline & -8 & -1 . & & \\
\hline & & -0 . & & \\
\hline & & & & \\
\hline & -5 & -2 . & & \\
\hline & & & & \\
\hline & -6 & -3 & 0 & \\
\hline & & & & \\
\hline & -3 & -3 & & \\
\hline & -3 & -1 & & \\
\hline & -2 & -1 & & \\
\hline & -1 & -0. & & \\
\hline & & & & \\
\hline & -4 & 0 . & & \\
\hline & & & & \\
\hline & -4 & 1. & & \\
\hline & & 2 . & & \\
\hline & -3 & & & \\
\hline & -3 & & & \\
\hline & -2 & & & \\
\hline & -4 . & 3. & & \\
\hline & & & & \\
\hline & -2. & 0 . & & \\
\hline & & . & & \\
\hline & -4 & -2 . & & \\
\hline & -5 & -2 . & & \\
\hline & & & & \\
\hline & -2 . & -2 . & & \\
\hline & -2 & 3 & & \\
\hline & -2 . & -4 . & & \\
\hline & & & & \\
\hline & -5 & -4 & & \\
\hline & -5 & -5 . & & \\
\hline & & -3 & & \\
\hline & & & & \\
\hline & & & & \\
\hline & & & & \\
\hline & & & & \\
\hline & -2 . & -4 . & & \\
\hline & & & & \\
\hline & -1 & -3 & & \\
\hline & & -2 & & \\
\hline & & -2 . & & \\
\hline & & -1 & & \\
\hline & & & & \\
\hline & & -2 & & \\
\hline & & & & \\
\hline & -5 & -0 . & & \\
\hline-0. & -3 & 0.850 & 1.00 & \\
\hline
\end{tabular}




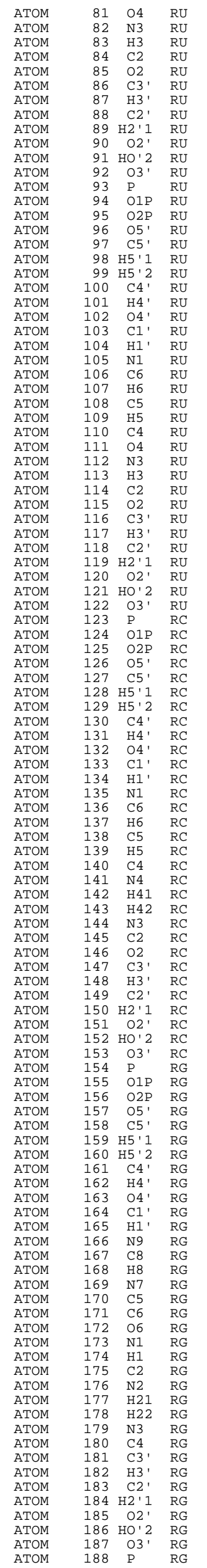

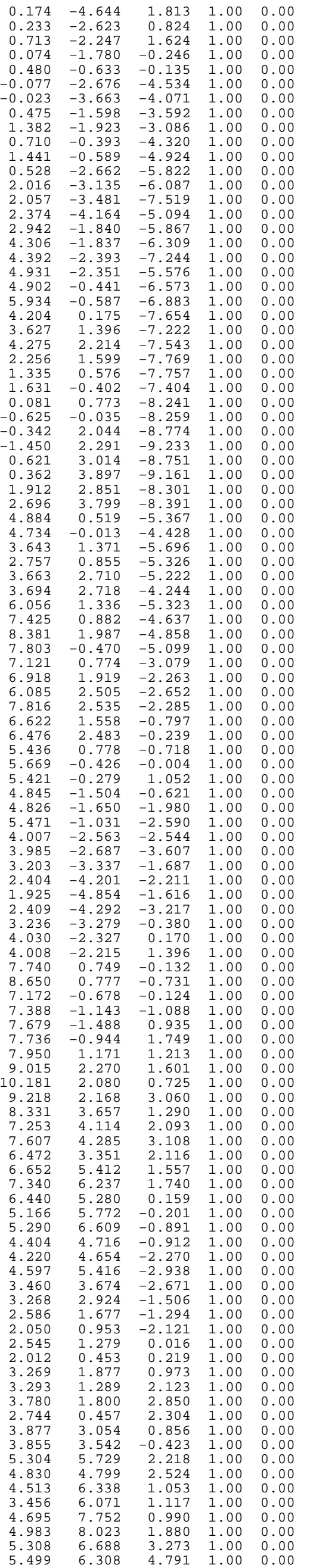




\begin{tabular}{|c|c|c|c|}
\hline ATOM & 189 & O1P & RG \\
\hline ATOM & 190 & $\mathrm{O} 2 \mathrm{P}$ & RG \\
\hline ATOM & 191 & $05^{\prime}$ & RG \\
\hline ATOM & 192 & C5' & RG \\
\hline ATOM & 193 & H5' 1 & RG \\
\hline ATOM & 194 & H5' 2 & RG \\
\hline ATOM & 195 & $\mathrm{C} 4{ }^{\prime}$ & RG \\
\hline ATOM & 196 & $\mathrm{H}_{4}{ }^{\prime}$ & RG \\
\hline ATOM & 197 & O4' & RG \\
\hline ATOM & 198 & $\mathrm{C} 1^{\prime}$ & RG \\
\hline ATOM & 199 & H1' & RG \\
\hline ATOM & 200 & N9 & RG \\
\hline ATOM & 201 & C8 & RG \\
\hline ATOM & 202 & H8 & RG \\
\hline ATOM & 203 & N7 & RG \\
\hline ATOM & 204 & C5 & RG \\
\hline ATOM & 205 & C6 & RG \\
\hline ATOM & 206 & 06 & RG \\
\hline ATOM & 207 & N1 & RG \\
\hline ATOM & 208 & $\mathrm{H} 1$ & RG \\
\hline ATOM & 209 & $\mathrm{C} 2$ & RG \\
\hline ATOM & 210 & N2 & RG \\
\hline ATOM & 211 & H 21 & RG \\
\hline ATOM & 212 & H22 & RG \\
\hline ATOM & 213 & N3 & RG \\
\hline ATOM & 214 & C4 & RG \\
\hline ATOM & 215 & C3' & RG \\
\hline ATOM & 216 & H3' & RG \\
\hline ATOM & 217 & C2' & RG \\
\hline ATOM & 218 & $\mathrm{H} 2{ }^{\prime} 1$ & RG \\
\hline ATOM & 219 & $02^{\prime}$ & RG \\
\hline ATOM & 220 & HO' 2 & RG \\
\hline ATOM & 221 & $03^{\prime}$ & RG \\
\hline ATOM & 222 & $\mathrm{P}$ & RU3 \\
\hline ATOM & 223 & $01 \mathrm{P}$ & RU3 \\
\hline ATOM & 224 & $\mathrm{O} 2 \mathrm{P}$ & RU3 \\
\hline ATOM & 225 & $05^{\prime}$ & RU3 \\
\hline ATOM & 226 & C5' & RU3 \\
\hline ATOM & 227 & H5' 1 & RU3 \\
\hline ATOM & 228 & H5' 2 & RU3 \\
\hline ATOM & 229 & C4 & RU3 \\
\hline ATOM & 230 & H4 ${ }^{\prime}$ & RU3 \\
\hline ATOM & 231 & O4' & RU3 \\
\hline ATOM & 232 & C1' & RU3 \\
\hline ATOM & 233 & H1 ' & RU3 \\
\hline ATOM & 234 & N1 & RU3 \\
\hline ATOM & 235 & $\mathrm{C} 6$ & RU3 \\
\hline ATOM & 236 & H6 & RU3 \\
\hline ATOM & 237 & C5 & RU3 \\
\hline ATOM & 238 & H5 & RU3 \\
\hline ATOM & 239 & C4 & RU3 \\
\hline ATOM & 240 & 04 & RU3 \\
\hline ATOM & 241 & N3 & RU3 \\
\hline ATOM & 242 & H3 & RU3 \\
\hline ATOM & 243 & $\mathrm{C} 2$ & RU3 \\
\hline ATOM & 244 & 02 & RU3 \\
\hline ATOM & 245 & C3' & RU3 \\
\hline ATOM & 246 & H3' & RU3 \\
\hline ATOM & 247 & C2' & RU3 \\
\hline ATOM & 248 & H2' 1 & RU3 \\
\hline ATOM & 249 & $02^{\prime}$ & RU3 \\
\hline ATOM & 250 & $\mathrm{HO}^{\prime} 2$ & RU3 \\
\hline ATOM & 251 & $03^{\prime}$ & RU3 \\
\hline ATOM & 252 & H3T & RU3 \\
\hline END & & & \\
\hline
\end{tabular}

\begin{tabular}{|c|c|c|c|c|}
\hline $\begin{array}{l}5.167 \\
6.838\end{array}$ & $\begin{array}{l}7.526 \\
5.725\end{array}$ & $\begin{array}{l}5.551 \\
4.959\end{array}$ & $\begin{array}{l}1.00 \\
1.00\end{array}$ & $\begin{array}{l}0.00 \\
0.00\end{array}$ \\
\hline 4.409 & 5.208 & 5.175 & 1.00 & 0.00 \\
\hline 3.033 & 5.529 & 5.371 & 1.00 & 0.00 \\
\hline 2.915 & 6.410 & 6.002 & 1.00 & 0.00 \\
\hline 2.564 & 4.698 & 5.894 & 1.00 & 0.00 \\
\hline 2.302 & 5.744 & 4.039 & 1.00 & 0.00 \\
\hline 2.639 & 6.676 & 3.583 & 1.00 & 0.00 \\
\hline 2.554 & 4.661 & 3.158 & 1.00 & 0.00 \\
\hline 1.340 & 4.198 & 2.600 & 1.00 & 0.00 \\
\hline 1.445 & 4.013 & 1.529 & 1.00 & 0.00 \\
\hline 0.945 & 2.943 & 3.284 & 1.00 & 0.00 \\
\hline 1.453 & 2.365 & 4.424 & 1.00 & 0.00 \\
\hline 2.246 & 2.816 & 5.004 & 1.00 & 0.00 \\
\hline 0.872 & 1.247 & 4.771 & 1.00 & 0.00 \\
\hline-0.029 & 1.018 & 3.719 & 1.00 & 0.00 \\
\hline-0.960 & -0.048 & 3.447 & 1.00 & 0.00 \\
\hline-1.232 & -1.046 & 4.108 & 1.00 & 0.00 \\
\hline-1.660 & 0.108 & 2.278 & 1.00 & 0.00 \\
\hline-2.288 & -0.627 & 2.007 & 1.00 & 0.00 \\
\hline-1.461 & 1.122 & 1.426 & 1.00 & 0.00 \\
\hline-2.229 & 1.155 & 0.389 & 1.00 & 0.00 \\
\hline-2.110 & 1.935 & -0.236 & 1.00 & 0.00 \\
\hline-2.842 & 0.373 & 0.204 & 1.00 & 0.00 \\
\hline-0.672 & 2.162 & 1.640 & 1.00 & 0.00 \\
\hline 0.044 & 2.037 & 2.795 & 1.00 & 0.00 \\
\hline 0.787 & 5.811 & 4.194 & 1.00 & 0.00 \\
\hline 0.489 & 5.096 & 4.963 & 1.00 & 0.00 \\
\hline 0.309 & 5.297 & 2.829 & 1.00 & 0.00 \\
\hline-0.694 & 4.878 & 2.899 & 1.00 & 0.00 \\
\hline 0.395 & 6.234 & 1.753 & 1.00 & 0.00 \\
\hline 0.795 & 7.067 & 2.081 & 1.00 & 0.00 \\
\hline 0.323 & 7.107 & 4.493 & 1.00 & 0.00 \\
\hline-0.982 & 7.299 & 5.342 & 1.00 & 0.00 \\
\hline-1.233 & 8.747 & 5.426 & 1.00 & 0.00 \\
\hline-0.811 & 6.539 & 6.595 & 1.00 & 0.00 \\
\hline-2.158 & 6.588 & 4.558 & 1.00 & 0.00 \\
\hline-2.715 & 7.085 & 3.351 & 1.00 & 0.00 \\
\hline-1.933 & 7.248 & 2.610 & 1.00 & 0.00 \\
\hline-3.229 & 8.029 & 3.536 & 1.00 & 0.00 \\
\hline-3.719 & 6.063 & 2.794 & 1.00 & 0.00 \\
\hline-4.113 & 6.427 & 1.845 & 1.00 & 0.00 \\
\hline-3.104 & 4.791 & 2.588 & 1.00 & 0.00 \\
\hline-4.059 & 3.762 & 2.817 & 1.00 & 0.00 \\
\hline-4.223 & 3.228 & 1.878 & 1.00 & 0.00 \\
\hline-3.628 & 2.782 & 3.859 & 1.00 & 0.00 \\
\hline-2.752 & 3.092 & 4.881 & 1.00 & 0.00 \\
\hline-2.288 & 4.072 & 4.903 & 1.00 & 0.00 \\
\hline-2.488 & 2.178 & 5.862 & 1.00 & 0.00 \\
\hline-1.809 & 2.415 & 6.668 & 1.00 & 0.00 \\
\hline-3.105 & 0.868 & 5.873 & 1.00 & 0.00 \\
\hline-2.973 & -0.020 & 6.709 & 1.00 & 0.00 \\
\hline-3.923 & 0.636 & 4.811 & 1.00 & 0.00 \\
\hline-4.366 & -0.268 & 4.764 & 1.00 & 0.00 \\
\hline-4.250 & 1.533 & 3.836 & 1.00 & 0.00 \\
\hline-5.114 & 1.211 & 3.026 & 1.00 & 0.00 \\
\hline & 5.799 & 3.724 & 1.00 & 0.00 \\
\hline-4.552 & 5.692 & 4.750 & 1.00 & 0.00 \\
\hline-5 & 4.433 & 3.221 & 1.00 & 0.00 \\
\hline-5.884 & 3.896 & 4.027 & 1.00 & 0.00 \\
\hline-6.207 & 4.494 & 2.062 & 1.00 & 0.00 \\
\hline-7.002 & 5.029 & 2.288 & 1.00 & 0.00 \\
\hline-5.928 & 6.786 & 3.667 & 1.00 & 0.00 \\
\hline-6.609 & 6.539 & 4.344 & 1.00 & 0.00 \\
\hline
\end{tabular}

\begin{tabular}{|c|c|c|c|}
\hline \multicolumn{4}{|c|}{$\mathrm{C}-80$ deg-QM.pdb } \\
\hline ATOM & 1 & H5T & RA5 \\
\hline ATOM & 2 & $05^{\prime}$ & RA5 \\
\hline ATOM & 3 & C5' & RA5 \\
\hline ATOM & 4 & H5' 1 & RA5 \\
\hline ATOM & 5 & H5' 2 & RA5 \\
\hline ATOM & 6 & C4 ${ }^{\prime}$ & RA5 \\
\hline ATOM & 7 & $\mathrm{H}_{4}{ }^{\prime}$ & RA5 \\
\hline ATOM & 8 & O $4^{\prime}$ & RA5 \\
\hline ATOM & 9 & $\mathrm{C} 1^{\prime}$ & RA5 \\
\hline $\mathrm{ATOM}$ & 10 & $\mathrm{H} 1{ }^{\prime}$ & RA5 \\
\hline $\mathrm{ATOM}$ & 11 & N9 & RA5 \\
\hline ATOM & 12 & C8 & RA5 \\
\hline ATOM & 13 & H8 & RA5 \\
\hline ATOM & 14 & N7 & RA5 \\
\hline ATOM & 15 & C5 & RA5 \\
\hline ATOM & 16 & C6 & RA5 \\
\hline ATOM & 17 & N6 & RA5 \\
\hline ATOM & 18 & H61 & RA5 \\
\hline ATOM & 19 & $\mathrm{H} 62$ & RA5 \\
\hline ATOM & 20 & N1 & RA5 \\
\hline ATOM & 21 & C2 & RA5 \\
\hline ATOM & 22 & $\mathrm{H} 2$ & RA5 \\
\hline ATOM & 23 & N3 & RA5 \\
\hline ATOM & 24 & C4 & RA5 \\
\hline ATOM & 25 & C3' & RA5 \\
\hline ATOM & 26 & H3' & RA5 \\
\hline ATOM & 27 & $\mathrm{C} 2{ }^{\prime}$ & RA5 \\
\hline ATOM & 28 & $\mathrm{H} 2 \mathrm{\prime}^{\prime} 1$ & RA5 \\
\hline ATOM & 29 & $02^{\prime}$ & RA5 \\
\hline ATOM & 30 & Hо' 2 & RA5 \\
\hline АТOM & 31 & $03^{\prime}$ & RA5 \\
\hline ATOM & 32 & P & $\mathrm{RC}$ \\
\hline ATOM & 33 & $\mathrm{O} 1 \mathrm{P}$ & $\mathrm{RC}$ \\
\hline ATOM & 34 & $\mathrm{O} 2 \mathrm{P}$ & $\mathrm{RC}$ \\
\hline АТOM & 35 & $05^{\prime}$ & $\mathrm{RC}$ \\
\hline ATOM & 36 & C5 ${ }^{\prime}$ & $\mathrm{RC}$ \\
\hline ATOM & 37 & H5' 1 & $\mathrm{RC}$ \\
\hline ATOM & 38 & H5 ' 2 & $\mathrm{RC}$ \\
\hline ATOM & 39 & C4 ${ }^{\prime}$ & $\mathrm{RC}$ \\
\hline ATOM & 40 & $\mathrm{H} 4{ }^{\prime}$ & $\mathrm{RC}$ \\
\hline & & & \\
\hline
\end{tabular}

$\begin{array}{rrrrrl}10.200 & 3.785 & -7.869 & 1.00 & 0.00 & \\ 10.076 & 3.323 & -7.022 & 1.00 & 0.00 & \mathrm{H} \\ 11.270 & 2.705 & -6.631 & 1.00 & 0.00 & \mathrm{O} \\ 12.135 & 3.376 & -6.710 & 1.00 & 0.00 & \mathrm{C} \\ 11.515 & 1.819 & -7.232 & 1.00 & 0.00 & \mathrm{H} \\ 11.154 & 2.250 & -5.199 & 1.00 & 0.00 & \mathrm{H} \\ 12.123 & 1.819 & -4.919 & 1.00 & 0.00 & \mathrm{C} \\ 10.865 & 3.371 & -4.352 & 1.00 & 0.00 & \mathrm{H} \\ 10.025 & 3.004 & -3.296 & 1.00 & 0.00 & \mathrm{O} \\ 10.451 & 3.302 & -2.336 & 1.00 & 0.00 & \mathrm{C} \\ 8.745 & 3.695 & -3.417 & 1.00 & 0.00 & \mathrm{H} \\ 8.284 & 4.447 & -4.459 & 1.00 & 0.00 & \mathrm{~N} \\ 8.773 & 4.449 & -5.420 & 1.00 & 0.00 & \mathrm{C} \\ 7.206 & 5.114 & -4.156 & 1.00 & 0.00 & \mathrm{H} \\ 6.938 & 4.790 & -2.851 & 1.00 & 0.00 & \mathrm{~N} \\ 5.941 & 5.203 & -1.940 & 1.00 & 0.00 & \mathrm{C} \\ 5.051 & 6.146 & -2.206 & 1.00 & 0.00 & \mathrm{C} \\ 4.426 & 6.470 & -1.482 & 1.00 & 0.00 & \mathrm{~N} \\ 5.150 & 6.711 & -3.038 & 1.00 & 0.00 & \mathrm{H} \\ 5.917 & 4.595 & -0.742 & 1.00 & 0.00 & \mathrm{H} \\ 6.882 & 3.736 & -0.429 & 1.00 & 0.00 & \mathrm{~N} \\ 6.831 & 3.299 & 0.557 & 1.00 & 0.00 & \mathrm{C} \\ 7.917 & 3.364 & -1.159 & 1.00 & 0.00 & \mathrm{H} \\ 7.881 & 3.903 & -2.378 & 1.00 & 0.00 & \mathrm{~N} \\ 10.043 & 1.254 & -4.871 & 1.00 & 0.00 & \mathrm{C} \\ 9.123 & 1.543 & -5.396 & 1.00 & 0.00 & \mathrm{C} \\ 9.884 & 1.482 & -3.364 & 1.00 & 0.00 & \mathrm{H} \\ 8.927 & 1.154 & -2.960 & 1.00 & 0.00 & \mathrm{C} \\ 10.960 & 0.923 & -2.653 & 1.00 & 0.00 & \mathrm{H} \\ 10.698 & 0.126 & -2.181 & 1.00 & 0.00 & \mathrm{O} \\ 10.427 & -0.055 & -5.206 & 1.00 & 0.00 & \mathrm{H} \\ 9.311 & -1.165 & -5.594 & 1.00 & 0.00 & \mathrm{O} \\ 10.087 & -2.411 & -5.925 & 1.00 & 0.00 & \mathrm{P} \\ 8.311 & -0.609 & -6.560 & 1.00 & 0.00 & \mathrm{O} \\ 8.509 & -1.403 & -4.203 & 1.00 & 0.00 & \mathrm{O} \\ 9.082 & -2.227 & -3.208 & 1.00 & 0.00 & \mathrm{O} \\ 10.035 & -1.821 & -2.853 & 1.00 & 0.00 & \mathrm{C} \\ 9.290 & -3.225 & -3.592 & 1.00 & 0.00 & \mathrm{H} \\ 8.153 & -2.358 & -2.036 & 1.00 & 0.00 & \mathrm{H} \\ 8.655 & -3.017 & -1.320 & 1.00 & 0.00 & \mathrm{O} \\ 7.932 & -1.077 & -1.451 & 1.00 & 0.00 & \end{array}$




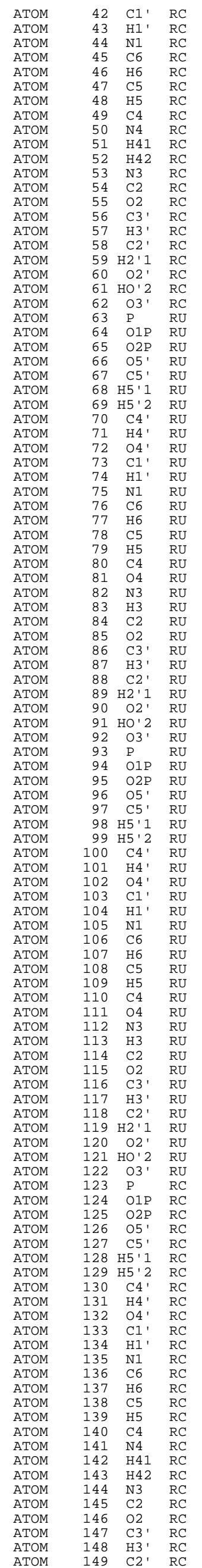

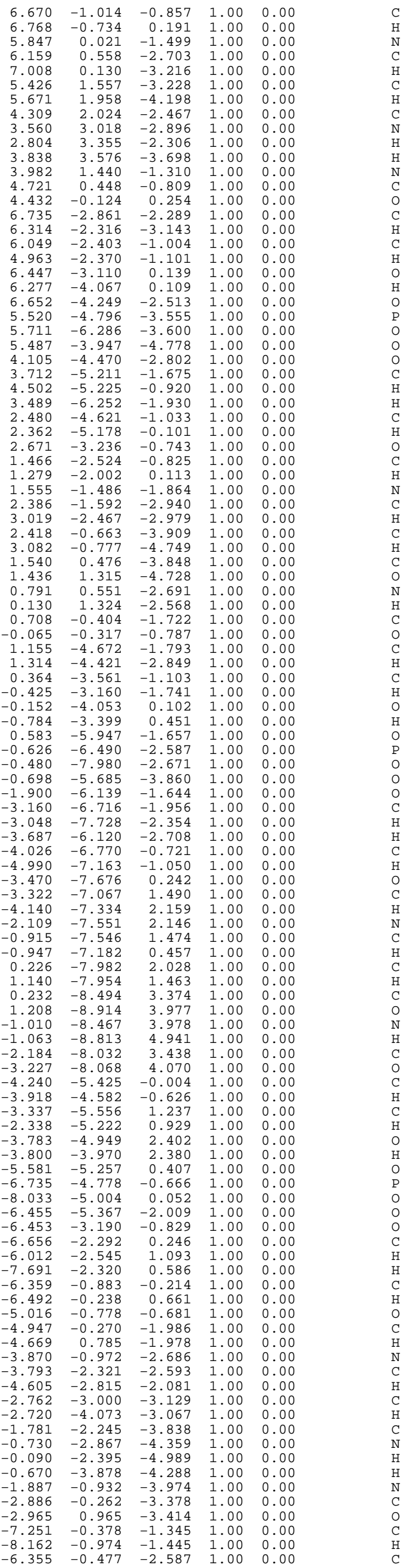




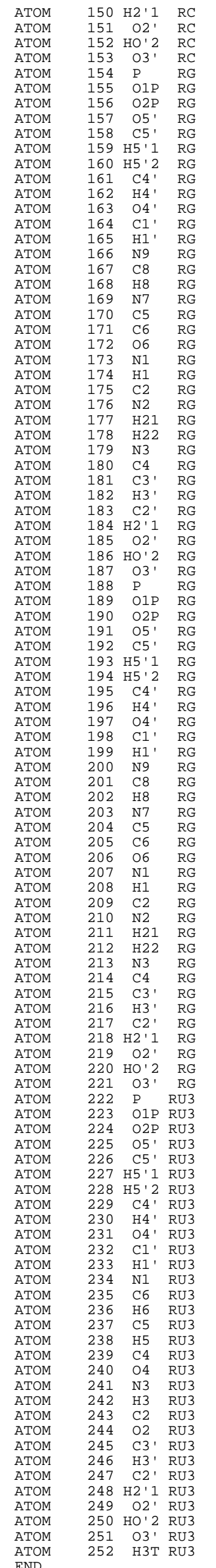

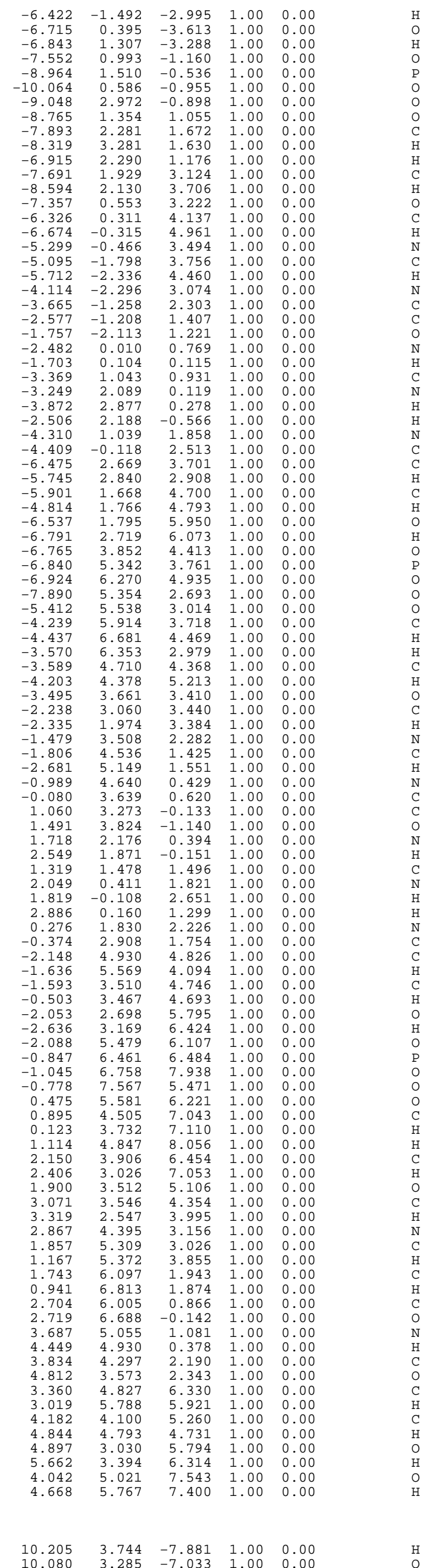

$\begin{array}{lll}\text { C-180 deg-QM.pdb } & \\ \text { ATOM } & 1 & \text { H5T RA5 }\end{array}$

$\begin{array}{llll}\text { ATOM } & 1 & \text { H5T } & \text { RA5 } \\ \text { ATOM } & 2 & \text { O5 } & \text { RA5 }\end{array}$

$\begin{array}{lllll}10.080 & 3.285 & -7.033 & 1.00 & 0.00\end{array}$ 


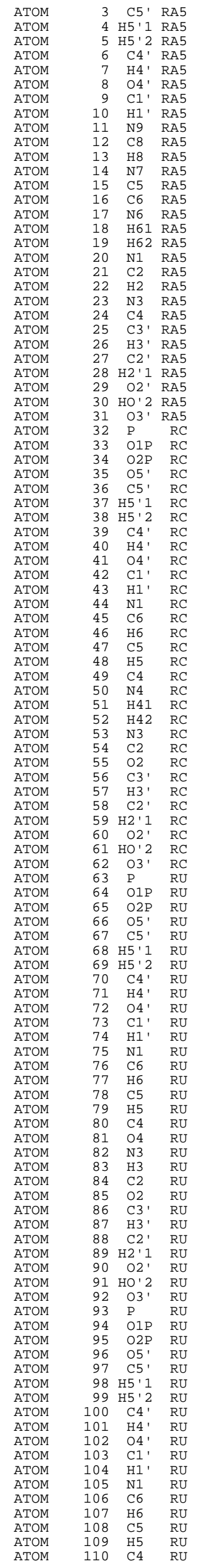

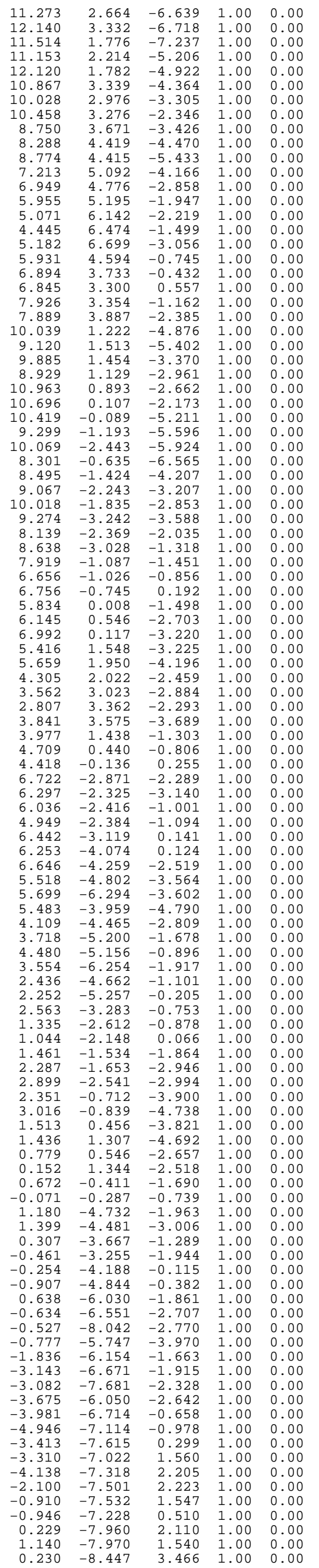




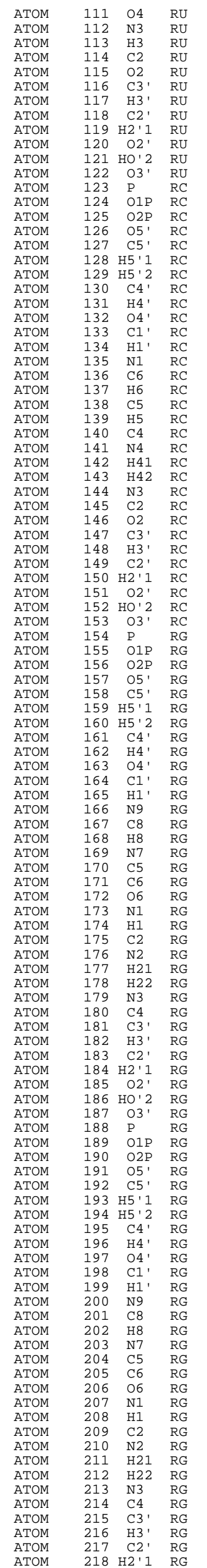

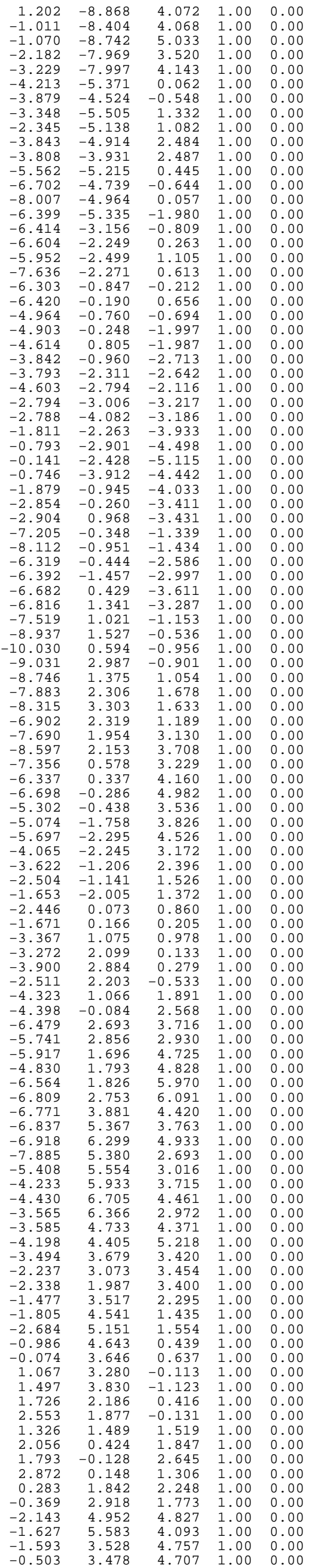




\begin{tabular}{|c|c|c|c|}
\hline ATOM & 219 & $\mathrm{O} 2^{\prime}$ & RG \\
\hline ATOM & 220 & HO' 2 & RG \\
\hline ATOM & 221 & O3' & RG \\
\hline ATOM & 222 & P & RU3 \\
\hline ATOM & 223 & O1P & RU3 \\
\hline ATOM & 224 & $\mathrm{O} 2 \mathrm{P}$ & RU3 \\
\hline ATOM & 225 & $05^{\prime}$ & RU3 \\
\hline ATOM & 226 & C5' & RU3 \\
\hline ATOM & 227 & H5' 1 & RU3 \\
\hline ATOM & 228 & H5' 2 & RU3 \\
\hline ATOM & 229 & C4 ' & RU3 \\
\hline ATOM & 230 & H4 ' & RU3 \\
\hline ATOM & 231 & $04{ }^{\prime}$ & RU3 \\
\hline ATOM & 232 & $\mathrm{C} 1{ }^{\prime}$ & RU3 \\
\hline ATOM & 233 & H1 ' & RU3 \\
\hline ATOM & 234 & N1 & RU3 \\
\hline ATOM & 235 & $\mathrm{C} 6$ & RU3 \\
\hline ATOM & 236 & H6 & RU3 \\
\hline ATOM & 237 & C5 & RU3 \\
\hline ATOM & 238 & H5 & RU3 \\
\hline ATOM & 239 & C4 & RU3 \\
\hline ATOM & 240 & 04 & RU3 \\
\hline ATOM & 241 & N3 & RU3 \\
\hline ATOM & 242 & H3 & RU3 \\
\hline ATOM & 243 & $\mathrm{C} 2$ & RU3 \\
\hline ATOM & 244 & 02 & RU3 \\
\hline ATOM & 245 & C3' & RU3 \\
\hline ATOM & 246 & H3' & RU3 \\
\hline ATOM & 247 & C2' & RU3 \\
\hline ATOM & 248 & $\mathrm{H} 2{ }^{\prime} 1$ & RU3 \\
\hline ATOM & 249 & $02^{\prime}$ & RU3 \\
\hline ATOM & 250 & HO' 2 & RU3 \\
\hline ATOM & 251 & O3' & RU3 \\
\hline ATOM & 252 & H3T & RU3 \\
\hline END & & & \\
\hline
\end{tabular}

$\begin{array}{rrrrr}-2.059 & 2.726 & 5.810 & 1.00 & 0.00 \\ -2.641 & 3.204 & 6.436 & 1.00 & 0.00 \\ -2.078 & 5.510 & 6.106 & 1.00 & 0.00 \\ -0.831 & 6.485 & 6.477 & 1.00 & 0.00 \\ -1.025 & 6.787 & 7.931 & 1.00 & 0.00 \\ -0.758 & 7.590 & 5.461 & 1.00 & 0.00 \\ 0.486 & 5.599 & 6.213 & 1.00 & 0.00 \\ 0.906 & 4.524 & 7.037 & 1.00 & 0.00 \\ 0.136 & 3.751 & 7.106 & 1.00 & 0.00 \\ 1.127 & 4.868 & 8.049 & 1.00 & 0.00 \\ 2.160 & 3.923 & 6.449 & 1.00 & 0.00 \\ 2.415 & 3.041 & 7.045 & 1.00 & 0.00 \\ 1.909 & 3.530 & 5.101 & 1.00 & 0.00 \\ 3.081 & 3.557 & 4.351 & 1.00 & 0.00 \\ 3.323 & 2.556 & 3.992 & 1.00 & 0.00 \\ 2.881 & 4.407 & 3.153 & 1.00 & 0.00 \\ 1.871 & 5.320 & 3.020 & 1.00 & 0.00 \\ 1.177 & 5.383 & 3.845 & 1.00 & 0.00 \\ 1.760 & 6.107 & 1.935 & 1.00 & 0.00 \\ 0.958 & 6.822 & 1.862 & 1.00 & 0.00 \\ 2.726 & 6.015 & 0.862 & 1.00 & 0.00 \\ 2.746 & 6.697 & -0.146 & 1.00 & 0.00 \\ 3.708 & 5.065 & 1.081 & 1.00 & 0.00 \\ 4.469 & 4.938 & 0.377 & 1.00 & 0.00 \\ 3.851 & 4.307 & 2.190 & 1.00 & 0.00 \\ 4.826 & 3.581 & 2.345 & 1.00 & 0.00 \\ 3.372 & 4.841 & 6.324 & 1.00 & 0.00 \\ 3.037 & 5.803 & 5.913 & 1.00 & 0.00 \\ 4.193 & 4.108 & 5.256 & 1.00 & 0.00 \\ 4.858 & 4.797 & 4.727 & 1.00 & 0.00 \\ 4.904 & 3.036 & 5.792 & 1.00 & 0.00 \\ 5.673 & 3.400 & 6.307 & 1.00 & 0.00 \\ 4.054 & 5.034 & 7.538 & 1.00 & 0.00 \\ 4.681 & 5.779 & 7.396 & 1.00 & 0.00 \\ & & & & \end{array}$

D-80deg-MM.pdb

ATOM

$\begin{array}{llllll}-13.365 & 0.141 & -0.378 & 1.00 & 0.00\end{array}$ $\begin{array}{lllll}-13.254 & -0.819 & -0.584 & 1.00 & 0.00\end{array}$

$\begin{array}{lllll}-13.423 & -0.951 & -1.985 & 1.00 & 0.00\end{array}$

$\begin{array}{lllll}-14.281 & -0.357 & -2.304 & 1.00 & 0.00\end{array}$

$\begin{array}{lllll}-13.624 & -1.996 & -2.227 & 1.00 & 0.00\end{array}$

$\begin{array}{lllll}-12.193 & -0.495 & -2.778 & 1.00 & 0.00\end{array}$

$\begin{array}{lllll}-12.437 & -0.560 & -3.841 & 1.00 & 0.00\end{array}$

$\begin{array}{lllll}-11.810 & 0.845 & -2.465 & 1.00 & 0.00\end{array}$

$\begin{array}{lllll}-10.390 & 0.952 & -2.552 & 1.00 & 0.00\end{array}$

$\begin{array}{rrrrr}-10.110 & 1.763 & -3.231 & 1.00 & 0.00 \\ -9.764 & 1.205 & -1.229 & 1.00 & 0.00\end{array}$

$\begin{array}{rrrrr}-9.764 & 1.205 & -1.229 & 1.00 & 0.00 \\ -10.245 & 0.940 & 0.034 & 1.00 & 0.00\end{array}$

$\begin{array}{lllll}-11.205 & 0.472 & 0.204 & 1.00 & 0.00\end{array}$

$\begin{array}{lllll}-9.463 & 1.339 & 1.008 & 1.00 & 0.00 \\ -8.356 & 1.874 & 0.314 & 1.00 & 0.00\end{array}$

$\begin{array}{lllll}-7.138 & 2.505 & 0.675 & 1.00 & 0.00\end{array}$

$\begin{array}{lllll}-6.755 & 2.758 & 1.903 & 1.00 & 0.00\end{array}$

$\begin{array}{lllll}-5.925 & 3.318 & 2.047 & 1.00 & 0.00\end{array}$

$\begin{array}{lllll}-7.319 & 2.441 & 2.675 & 1.00 & 0.00\end{array}$

$\begin{array}{lllll}-6.262 & 2.935 & -0.226 & 1.00 & 0.00\end{array}$

$\begin{array}{lllll}-6.570 & 2.753 & -1.500 & 1.00 & 0.00\end{array}$

$\begin{array}{lllll}-5.834 & 3.096 & -2.214 & 1.00 & 0.00 \\ -7.644 & 2.156 & -2.009 & 1.00 & 0.00\end{array}$

$\begin{array}{lllll}-7.644 & 2.156 & -2.009 & 1.00 & 0.00 \\ -8.522 & 1.768 & -1.040 & 1.00 & 0.00\end{array}$

$\begin{array}{lllll}-10.945 & -1.340 & -2.541 & 1.00 & 0.00 \\ -10.803 & -1.454 & -1.467 & 1.00 & 0.00\end{array}$

$\begin{array}{lllll}-9.892 & -0.380 & -3.112 & 1.00 & 0.00\end{array}$

$\begin{array}{lllll}-8.900 & -0.621 & -2.736 & 1.00 & 0.00\end{array}$

$\begin{array}{lllll}-9.886 & -0.256 & -4.533 & 1.00 & 0.00\end{array}$

$\begin{array}{lllll}-10.368 & -1.005 & -4.941 & 1.00 & 0.00\end{array}$

$\begin{array}{lllll}-10.998 & -2.599 & -3.190 & 1.00 & 0.00\end{array}$

$\begin{array}{lllll}-10.048 & -3.795 & -2.787 & 1.00 & 0.00\end{array}$

$\begin{array}{lllll}-10.379 & -4.906 & -3.698 & 1.00 & 0.00\end{array}$

$\begin{array}{lllll}-10.226 & -4.004 & -1.340 & 1.00 & 0.00\end{array}$

$\begin{array}{lllll}-8.542 & -3.320 & -3.070 & 1.00 & 0.00\end{array}$

$\begin{array}{lllll}-8.089 & -3.139 & -4.411 & 1.00 & 0.00\end{array}$

$\begin{array}{lllll}-8.848 & -2.605 & -4.982 & 1.00 & 0.00\end{array}$

$\begin{array}{lllll}-7.959 & -4.117 & -4.878 & 1.00 & 0.00\end{array}$

$\begin{array}{lllll}-7.959 & -4.117 & -4.878 & 1.00 & 0.00 \\ -6.777 & -2.354 & -4.572 & 1.00 & 0.00\end{array}$

$\begin{array}{lllll}-6.777 & -2.354 & -4.572 & 1.00 & 0.00 \\ -6.621 & -2.214 & -5.641 & 1.00 & 0.00\end{array}$

$\begin{array}{lllll}-6.621 & -2.214 & -5.641 & 1.00 & 0.00 \\ -6.782 & -1.072 & -3.945 & 1.00 & 0.00\end{array}$

$\begin{array}{lllll}-5.449 & -0.732 & -3.547 & 1.00 & 0.00\end{array}$

$\begin{array}{lllll}-5.150 & 0.219 & -3.993 & 1.00 & 0.00\end{array}$

$\begin{array}{lllll}-5.319 & -0.641 & -2.063 & 1.00 & 0.00 \\ -6.345 & -1.023 & -1.234 & 1.00 & 0.00\end{array}$

$\begin{array}{lllll}-6.345 & -1.023 & -1.234 & 1.00 & 0.00 \\ -7.254 & -1.448 & -1.640 & 1.00 & 0.00\end{array}$

$\begin{array}{lllll}-6.216 & -0.864 & 0.101 & 1.00 & 0.00\end{array}$

$\begin{array}{lllll}-7.008 & -1.142 & 0.770 & 1.00 & 0.00\end{array}$

$\begin{array}{lllll}-5.018 & -0.313 & 0.582 & 1.00 & 0.00\end{array}$

$\begin{array}{lllll}-4.884 & -0.185 & 1.852 & 1.00 & 0.00\end{array}$

$\begin{array}{lllll}-4.050 & 0.258 & 2.204 & 1.00 & 0.00\end{array}$

$\begin{array}{lllll}-5.630 & -0.489 & 2.451 & 1.00 & 0.00\end{array}$

$\begin{array}{lllll}-4.151 & -0.086 & -1.508 & 1.00 & 0.00\end{array}$

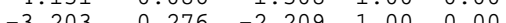

$\begin{array}{llll}-3.203 & 0.276-2.209-1.00 & 0.00\end{array}$

$\begin{array}{lllll}-5.527 & -3.032 & -4.036 & 1.00 & 0.00 \\ -5.696 & -3.293 & -2.994 & 1.00 & 0.00\end{array}$

$\begin{array}{lllll}-5.696 & -3.293 & -2.994 & 1.00 & 0.00 \\ -4.555 & -1.845 & -4.102 & 1.00 & 0.00\end{array}$

$\begin{array}{lllll}-4.555 & -1.845 & -4.102 & 1.00 & 0.00 \\ -3.682 & -2.015 & -3.474 & 1.00 & 0.00\end{array}$

$\begin{array}{lllll}-3.682 & -2.015 & -3.474 & 1.00 & 0.00 \\ -4.192 & -1.431 & -5.418 & 1.00 & 0.00\end{array}$

$\begin{array}{lllll}-3.259 & -1.656 & -5.604 & 1.00 & 0.00\end{array}$

$\begin{array}{lllll}-5.139 & -4.167 & -4.788 & 1.00 & 0.00\end{array}$

$\begin{array}{lllll}-4.311 & -5.333 & -4.120 & 1.00 & 0.00\end{array}$

$\begin{array}{lllll}-3.976 & -6.297 & -5.189 & 1.00 & 0.00\end{array}$

$\begin{array}{lllll}-5.041 & -5.799 & -2.922 & 1.00 & 0.00\end{array}$

$\begin{array}{lllll}-2.974 & -4.657 & -3.612 & 1.00 & 0.00\end{array}$

$\begin{array}{lllll}-1.946 & -4.269 & -4.503 & 1.00 & 0.00\end{array}$

$\begin{array}{lllll}-1.520 & -3.612 & -5.269 & 1.00 & 0.00\end{array}$

$\begin{array}{lllll}-0.828 & -3.518 & -3.780 & 1.00 & 0.00\end{array}$

$\begin{array}{lllll}-0.125 & -3.182 & -4.540 & 1.00 & 0.00\end{array}$ 


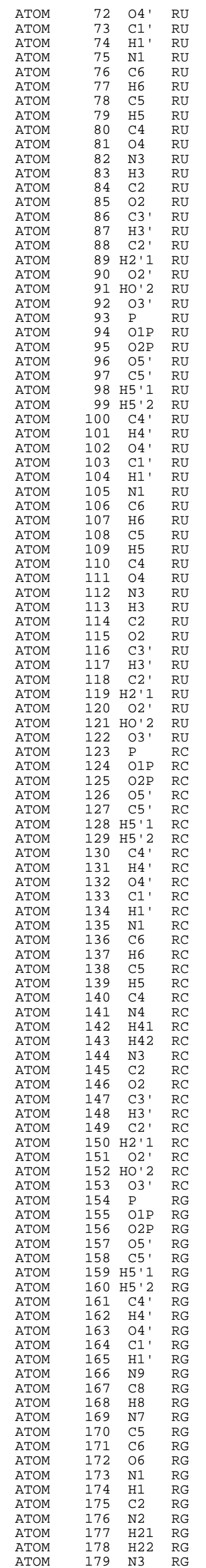

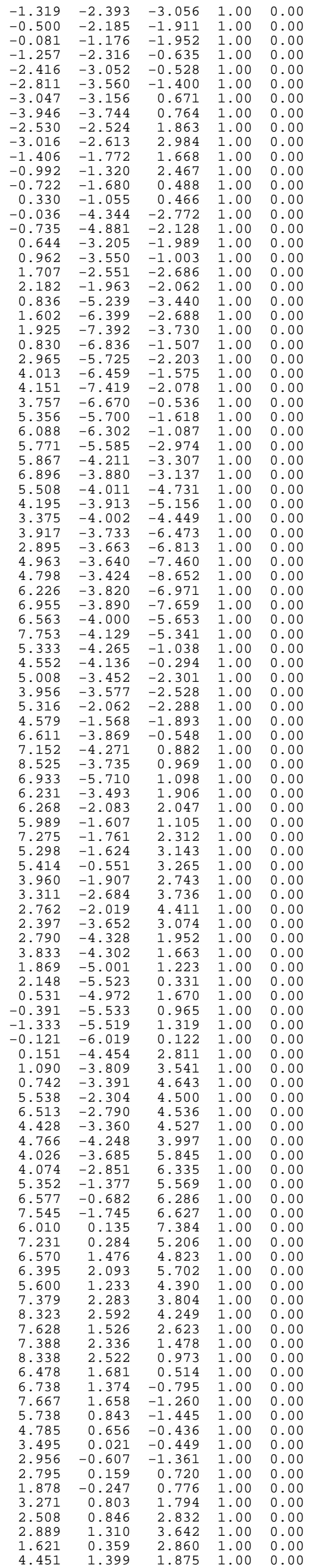




\begin{tabular}{|c|c|c|c|}
\hline ATOM & 180 & C4 & RG \\
\hline ATOM & 181 & C3' & RG \\
\hline ATOM & 182 & H3 ' & RG \\
\hline ATOM & 183 & C2 ${ }^{\prime}$ & RG \\
\hline ATOM & 184 & H2' 1 & RG \\
\hline ATOM & 185 & $\mathrm{O} 2^{\prime}$ & RG \\
\hline ATOM & 186 & HO' 2 & RG \\
\hline ATOM & 187 & $03^{\prime}$ & RG \\
\hline ATOM & 188 & P & RG \\
\hline ATOM & 189 & O1P & RG \\
\hline ATOM & 190 & $\mathrm{O} 2 \mathrm{P}$ & RG \\
\hline ATOM & 191 & $05^{\prime}$ & RG \\
\hline ATOM & 192 & C5' & RG \\
\hline ATOM & 193 & H5' 1 & RG \\
\hline ATOM & 194 & H5' 2 & RG \\
\hline ATOM & 195 & C4 & RG \\
\hline ATOM & 196 & H4 ' & RG \\
\hline ATOM & 197 & $04^{\prime}$ & RG \\
\hline ATOM & 198 & $\mathrm{C} 1^{\prime}$ & RG \\
\hline ATOM & 199 & H1 ${ }^{\prime}$ & RG \\
\hline ATOM & 200 & N9 & RG \\
\hline ATOM & 201 & C8 & RG \\
\hline ATOM & 202 & H8 & RG \\
\hline ATOM & 203 & N7 & RG \\
\hline ATOM & 204 & C5 & RG \\
\hline ATOM & 205 & C6 & RG \\
\hline ATOM & 206 & 06 & RG \\
\hline ATOM & 207 & N1 & RG \\
\hline ATOM & 208 & H1 & RG \\
\hline ATOM & 209 & $\mathrm{C} 2$ & RG \\
\hline ATOM & 210 & $\mathrm{~N} 2$ & RG \\
\hline ATOM & 211 & H 21 & RG \\
\hline ATOM & 212 & H 22 & RG \\
\hline ATOM & 213 & N3 & RG \\
\hline ATOM & 214 & C4 & RG \\
\hline ATOM & 215 & C3' & RG \\
\hline ATOM & 216 & H3' & RG \\
\hline ATOM & 217 & $\mathrm{C} 2{ }^{\prime}$ & RG \\
\hline ATOM & 218 & $\mathrm{H} 2{ }^{\prime} 1$ & RG \\
\hline ATOM & 219 & $02^{\prime}$ & RG \\
\hline ATOM & 220 & HO' 2 & RG \\
\hline ATOM & 221 & $03^{\prime}$ & RG \\
\hline ATOM & 222 & P & RU3 \\
\hline ATOM & 223 & $\mathrm{O} 1 \mathrm{P}$ & RU3 \\
\hline ATOM & 224 & $\mathrm{O} 2 \mathrm{P}$ & RU3 \\
\hline ATOM & 225 & $05^{\prime}$ & RU3 \\
\hline ATOM & 226 & C5 ' & RU3 \\
\hline ATOM & 227 & H5' 1 & RU3 \\
\hline ATOM & 228 & H5' 2 & RU3 \\
\hline ATOM & 229 & $\mathrm{C} 4{ }^{\prime}$ & RU3 \\
\hline ATOM & 230 & H4 ' & RU3 \\
\hline ATOM & 231 & $04^{\prime}$ & RU3 \\
\hline ATOM & 232 & $\mathrm{C} 1^{\prime}$ & RU3 \\
\hline ATOM & 233 & H1' & RU3 \\
\hline ATOM & 234 & N1 & RU3 \\
\hline ATOM & 235 & C6 & RU3 \\
\hline ATOM & 236 & H 6 & RU3 \\
\hline ATOM & 237 & C5 & RU3 \\
\hline ATOM & 238 & H5 & RU3 \\
\hline ATOM & 239 & C4 & RU3 \\
\hline ATOM & 240 & 04 & RU3 \\
\hline ATOM & 241 & N3 & RU3 \\
\hline ATOM & 242 & H3 & RU3 \\
\hline ATOM & 243 & $\mathrm{C} 2$ & RU3 \\
\hline ATOM & 244 & $\mathrm{O} 2$ & RU3 \\
\hline ATOM & 245 & C3' & RU3 \\
\hline ATOM & 246 & H3' & RU3 \\
\hline ATOM & 247 & C2' & RU3 \\
\hline ATOM & 248 & H2' 1 & RU3 \\
\hline ATOM & 249 & $02^{\prime}$ & RU3 \\
\hline ATOM & 250 & HO' 2 & RU3 \\
\hline ATOM & 251 & $03^{\prime}$ & RU3 \\
\hline ATOM & 252 & H3T & RU3 \\
\hline
\end{tabular}

\begin{tabular}{|c|c|c|c|c|}
\hline 5.178 & 1.282 & 0.725 & 1.00 & 0.00 \\
\hline 6.591 & 3.536 & 3.416 & 1.00 & 0.00 \\
\hline 5.536 & 3.338 & 3.567 & 1.00 & 0.00 \\
\hline 6.827 & 3.688 & 1.907 & 1.00 & 0.00 \\
\hline 5.867 & 3.888 & 1.423 & 1.00 & 0.00 \\
\hline 7.777 & 4.689 & 1.540 & 1.00 & 0.00 \\
\hline 7.630 & 5.449 & 2.135 & 1.00 & 0.00 \\
\hline 6.985 & 4.740 & 4.056 & 1.00 & 0.00 \\
\hline 6.483 & 5.204 & 5.479 & 1.00 & 0.00 \\
\hline 6.715 & 6.662 & 5.477 & 1.00 & 0.00 \\
\hline 7.132 & 4.377 & 6.517 & 1.00 & 0.00 \\
\hline 4.905 & 4.949 & 5.558 & 1.00 & 0.00 \\
\hline 3.935 & 5.810 & 4.964 & 1.00 & 0.00 \\
\hline 4.194 & 6.860 & 5.110 & 1.00 & 0.00 \\
\hline 2.983 & 5.630 & 5.462 & 1.00 & 0.00 \\
\hline 3.742 & 5.521 & 3.471 & 1.00 & 0.00 \\
\hline 4.670 & 5.731 & 2.941 & 1.00 & 0.00 \\
\hline 3.368 & 4.165 & 3.266 & 1.00 & 0.00 \\
\hline 2.429 & 4.080 & 2.203 & 1.00 & 0.00 \\
\hline 2.767 & 3.382 & 1.435 & 1.00 & 0.00 \\
\hline 1.127 & 3.613 & 2.713 & 1.00 & 0.00 \\
\hline 0.654 & 3.626 & 4.000 & 1.00 & 0.00 \\
\hline 1.197 & 4.086 & 4.815 & 1.00 & 0.00 \\
\hline-0.502 & 3.042 & 4.157 & 1.00 & 0.00 \\
\hline-0.805 & 2.587 & 2.866 & 1.00 & 0.00 \\
\hline-1.914 & 1.835 & 2.351 & 1.00 & 0.00 \\
\hline-2.882 & 1.373 & 2.939 & 1.00 & 0.00 \\
\hline-1.873 & 1.622 & 0.998 & 1.00 & 0.00 \\
\hline-2.619 & 1.100 & 0.578 & 1.00 & 0.00 \\
\hline-0.847 & 1.994 & 0.227 & 1.00 & 0.00 \\
\hline-0.938 & 1.634 & -1.011 & 1.00 & 0.00 \\
\hline-0.109 & 1.792 & -1.568 & 1.00 & 0.00 \\
\hline-1.757 & 1.141 & -1.336 & 1.00 & 0.00 \\
\hline 0.217 & 2.676 & 0.631 & 1.00 & 0.00 \\
\hline 0.173 & 2.958 & 1.972 & 1.00 & 0.00 \\
\hline 2.638 & 6.349 & 2.821 & 1.00 & 0.00 \\
\hline 1.778 & 6.370 & 3.494 & 1.00 & 0.00 \\
\hline 2.313 & 5.471 & 1.603 & 1.00 & 0.00 \\
\hline 1.304 & 5.668 & 1.233 & 1.00 & 0.00 \\
\hline 3.278 & 5.510 & 0.555 & 1.00 & 0.00 \\
\hline 2.892 & 5.040 & -0.219 & 1.00 & 0.00 \\
\hline 3.053 & 7.659 & 2.484 & 1.00 & 0.00 \\
\hline 1.982 & 8.796 & 2.235 & 1.00 & 0.00 \\
\hline 2.743 & 9.997 & 1.839 & 1.00 & 0.00 \\
\hline 1.139 & 8.869 & 3.442 & 1.00 & 0.00 \\
\hline 1.099 & 8.313 & 1.006 & 1.00 & 0.00 \\
\hline 1.612 & 8.280 & -0.322 & 1.00 & 0.00 \\
\hline 2.514 & 7.674 & -0.371 & 1.00 & 0.00 \\
\hline 1.869 & 9.293 & -0.641 & 1.00 & 0.00 \\
\hline 0.579 & 7.712 & -1.304 & 1.00 & 0.00 \\
\hline 1.023 & 7.665 & -2.299 & 1.00 & 0.00 \\
\hline 0.119 & 6.411 & -0.944 & 1.00 & 0.00 \\
\hline-1.265 & 6.307 & -1.263 & 1.00 & 0.00 \\
\hline-1.377 & 5.641 & -2.120 & 1.00 & 0.00 \\
\hline-2.040 & 5.774 & -0.109 & 1.00 & 0.00 \\
\hline-2.017 & 6.399 & 1.120 & 1.00 & 0.00 \\
\hline-1.287 & 7.182 & 1.310 & 1.00 & 0.00 \\
\hline-2.922 & 6.054 & 2.076 & 1.00 & 0.00 \\
\hline-2.933 & 6.561 & 3.027 & 1.00 & 0.00 \\
\hline-3.923 & 5.037 & 1.847 & 1.00 & 0.00 \\
\hline-4.832 & 4.733 & 2.604 & 1.00 & 0.00 \\
\hline-3.816 & 4.397 & 0.647 & 1.00 & 0.00 \\
\hline-4.543 & 3.739 & 0.415 & 1.00 & 0.00 \\
\hline-2937 & 4.725 & -0.350 & 1.00 & 0.00 \\
\hline-2.989 & 4.097 & -1.402 & 1.00 & 0.00 \\
\hline-0.635 & 8.632 & -1.374 & 1.00 & 0.00 \\
\hline-0.820 & 9.087 & -0.399 & 1.00 & 0.00 \\
\hline-1.797 & 7.684 & -1.694 & 1.00 & 0.00 \\
\hline-2.672 & 7.972 & -1.106 & 1.00 & 0.00 \\
\hline-2 & 7.648 & -3.074 & 1.00 & 0.00 \\
\hline-2.313 & 8.571 & -3.352 & 1.00 & 0.00 \\
\hline-0.429 & 9.645 & -2.349 & 1.00 & 0.00 \\
\hline-0.857 & 10.458 & -1.998 & 1.00 & 0.00 \\
\hline
\end{tabular}

D-180 deg-MM.pdb

\begin{tabular}{|c|c|c|c|}
\hline ATOM & 1 & $\mathrm{H} 5 \mathrm{~T}$ & RA5 \\
\hline ATOM & 2 & $05^{\prime}$ & RA5 \\
\hline ATOM & 3 & C5 ${ }^{\prime}$ & RA5 \\
\hline ATOM & 4 & H5'1 & RA5 \\
\hline ATOM & 5 & H5' 2 & RA5 \\
\hline ATOM & 6 & C4 ${ }^{\prime}$ & RA5 \\
\hline ATOM & 7 & H4 ${ }^{\prime}$ & RA5 \\
\hline ATOM & 8 & $04^{\prime}$ & RA5 \\
\hline ATOM & 9 & C1' & RA5 \\
\hline ATOM & 10 & H1 ' & RA5 \\
\hline ATOM & 11 & N9 & RA5 \\
\hline ATOM & 12 & C8 & RA5 \\
\hline ATOM & 13 & H8 & RA5 \\
\hline ATOM & 14 & N7 & RA5 \\
\hline ATOM & 15 & C5 & RA5 \\
\hline ATOM & 16 & C6 & RA5 \\
\hline ATOM & 17 & N6 & RA5 \\
\hline ATOM & 18 & H61 & RA5 \\
\hline ATOM & 19 & H 62 & RA5 \\
\hline ATOM & 20 & N1 & RA5 \\
\hline ATOM & 21 & $\mathrm{C} 2$ & RA5 \\
\hline ATOM & 22 & $\mathrm{H} 2$ & RA5 \\
\hline ATOM & 23 & N3 & RA5 \\
\hline ATOM & 24 & C4 & RA5 \\
\hline ATOM & 25 & C3' & RA5 \\
\hline ATOM & 26 & H3' & RA5 \\
\hline ATOM & 27 & $\mathrm{C} 2{ }^{\prime}$ & RA5 \\
\hline ATOM & 28 & H2' 1 & RA5 \\
\hline ATOM & 29 & $02^{\prime}$ & RA5 \\
\hline ATOM & 30 & HO' 2 & RA5 \\
\hline ATOM & 31 & $03^{\prime}$ & RA5 \\
\hline $\mathrm{IOM}$ & 32 & $P$ & RC \\
\hline
\end{tabular}

$\begin{array}{rrrrr}-13.367 & 0.134 & -0.395 & 1.00 & 0.00 \\ -13.253 & -0.826 & -0.602 & 1.00 & 0.00 \\ -13.420 & -0.956 & -2.004 & 1.00 & 0.00 \\ -14.278 & -0.364 & -2.323 & 1.00 & 0.00 \\ -13.617 & -2.001 & -2.247 & 1.00 & 0.00 \\ -12.190 & -0.496 & -2.794 & 1.00 & 0.00 \\ -12.431 & -0.561 & -3.857 & 1.00 & 0.00 \\ -11.809 & 0.845 & -2.480 & 1.00 & 0.00 \\ -10.389 & 0.954 & -2.564 & 1.00 & 0.00 \\ -10.110 & 1.767 & -3.241 & 1.00 & 0.00 \\ -9.765 & 1.204 & -1.240 & 1.00 & 0.00 \\ -10.250 & 0.941 & 0.022 & 1.00 & 0.00 \\ -11.211 & 0.476 & 0.190 & 1.00 & 0.00 \\ -9.468 & 1.337 & 0.998 & 1.00 & 0.00 \\ -8.359 & 1.871 & 0.305 & 1.00 & 0.00 \\ -7.141 & 2.500 & 0.669 & 1.00 & 0.00 \\ -6.759 & 2.751 & 1.898 & 1.00 & 0.00 \\ -5.928 & 3.308 & 2.043 & 1.00 & 0.00 \\ -7.325 & 2.436 & 2.669 & 1.00 & 0.00 \\ -6.263 & 2.928 & -0.231 & 1.00 & 0.00 \\ -6.568 & 2.747 & -1.506 & 1.00 & 0.00 \\ -5.831 & 3.089 & -2.219 & 1.00 & 0.00 \\ -7.644 & 2.153 & -2.016 & 1.00 & 0.00 \\ -8.523 & 1.766 & -1.048 & 1.00 & 0.00 \\ -10.940 & -1.338 & -2.555 & 1.00 & 0.00 \\ -10.798 & -1.452 & -1.481 & 1.00 & 0.00 \\ -9.890 & -0.376 & -3.126 & 1.00 & 0.00 \\ -8.896 & -0.616 & -2.753 & 1.00 & 0.00 \\ -9.886 & -0.250 & -4.548 & 1.00 & 0.00 \\ -10.364 & -1.002 & -4.956 & 1.00 & 0.00 \\ -10.991 & -2.598 & -3.203 & 1.00 & 0.00 \\ -10.036 & -3.791 & -2.800 & 1.00 & 0.00\end{array}$




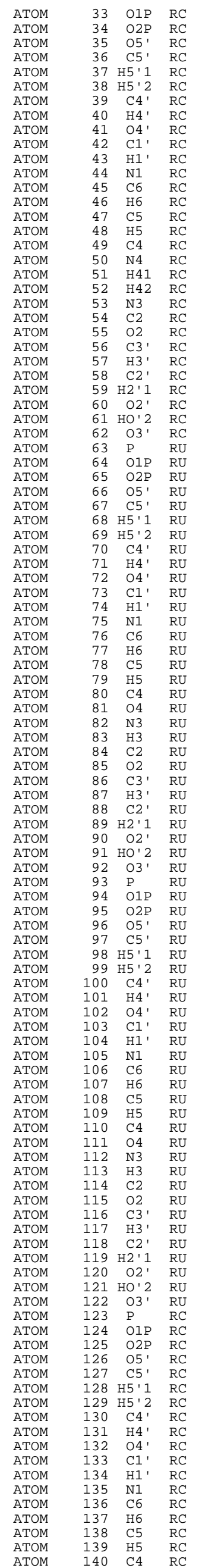

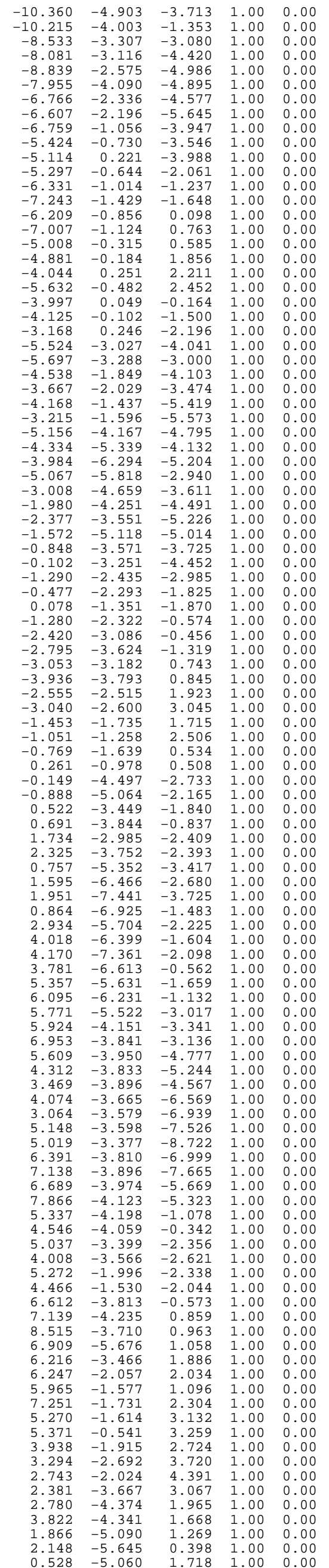




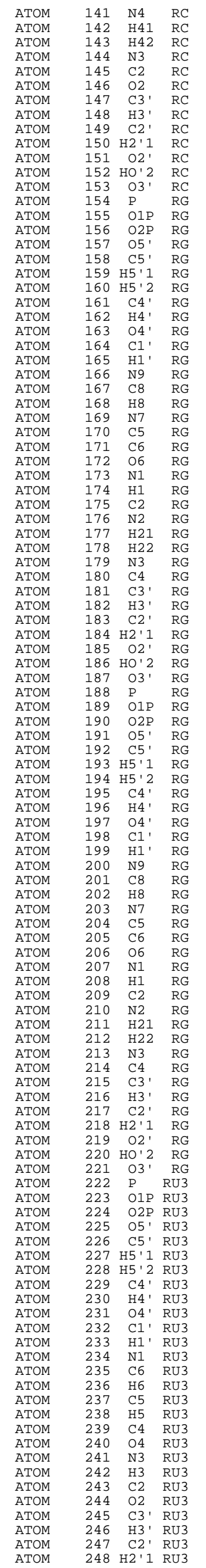

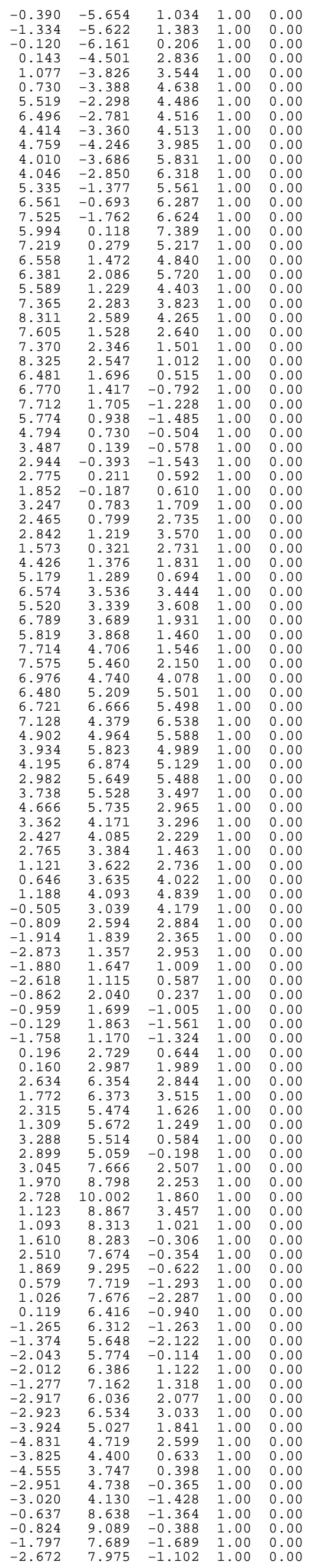




$\begin{array}{llrl}\text { ATOM } & 249 & \text { O2' } & \text { RU3 } \\ \text { ATOM } & 250 & \text { HO'2 } & \text { RU3 } \\ \text { ATOM } & 251 & \text { O3' } & \text { RU3 } \\ \text { ATOM } & 252 & \text { H3T } & \text { RU3 } \\ \text { END } & & & \end{array}$

$\mathrm{D}-80 \mathrm{deg}-\mathrm{QM} \cdot \mathrm{pdb}$

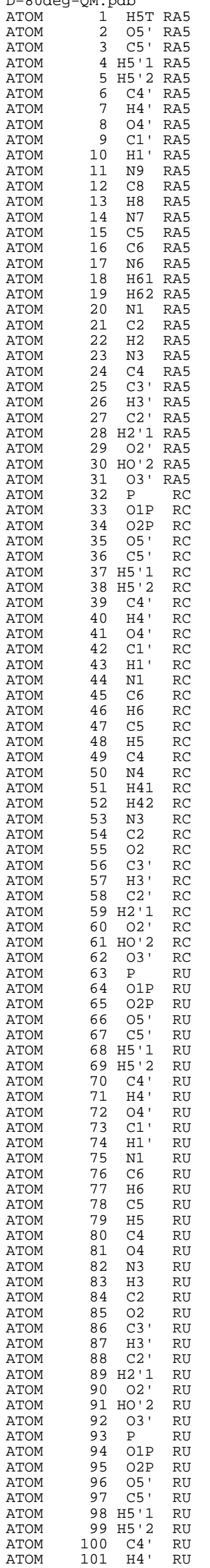

$\begin{array}{rrrrr}-2.135 & 7.657 & -3.070 & 1.00 & 0.00 \\ -2.313 & 8.580 & -3.346 & 1.00 & 0.00 \\ -0.430 & 9.653 & -2.336 & 1.00 & 0.00 \\ -0.863 & 10.464 & -1.986 & 1.00 & 0.00\end{array}$ $\begin{array}{llllll}-13.199 & -0.734 & -0.268 & 1.00 & 0.00\end{array}$ $\begin{array}{lllll}-13.345 & -0.841 & -1.661 & 1.00 & 0.00\end{array}$ $\begin{array}{lllll}-14.200 & -0.262 & -2.019 & 1.00 & 0.00\end{array}$ $\begin{array}{lllll}-13.572 & -1.882 & -1.906 & 1.00 & 0.00\end{array}$ $\begin{array}{lllll}-12.116 & -0.446 & -2.448 & 1.00 & 0.00\end{array}$ $\begin{array}{lllll}-12.400 & -0.505 & -3.508 & 1.00 & 0.00\end{array}$ $\begin{array}{lllll}-11.702 & 0.890 & -2.127 & 1.00 & 0.00\end{array}$ $\begin{array}{lllll}-10.308 & 1.009 & -2.205 & 1.00 & 0.00\end{array}$ $\begin{array}{lllll}-10.041 & 1.861 & -2.838 & 1.00 & 0.00\end{array}$ $\begin{array}{rrrrr}-9.697 & 1.264 & -0.897 & 1.00 & 0.00 \\ -10.150 & 1.029 & 0.366 & 1.00 & 0.00\end{array}$ $\begin{array}{lllll}-10.150 & 1.029 & 0.366 & 1.00 & 0.00 \\ -11.107 & 0.570 & 0.553 & 1.00 & 0.00\end{array}$ $\begin{array}{lllll}-11.107 & 0.570 & 0.553 & 1.00 & 0.00 \\ -9.315 & 1.422 & 1.294 & 1.00 & 0.00\end{array}$ $\begin{array}{lllll}-9.315 & 1.422 & 1.294 & 1.00 & 0.00 \\ -8.247 & 1.944 & 0.602 & 1.00 & 0.00\end{array}$ $\begin{array}{lllll}-7.045 & 2.568 & 1.007 & 1.00 & 0.00\end{array}$ $\begin{array}{lllll}-6.729 & 2.778 & 2.280 & 1.00 & 0.00\end{array}$ $\begin{array}{lllll}-5.946 & 3.375 & 2.539 & 1.00 & 0.00\end{array}$ $\begin{array}{lllll}-7.343 & 2.453 & 3.013 & 1.00 & 0.00\end{array}$ $\begin{array}{lllll}-6.199 & 2.962 & 0.044 & 1.00 & 0.00\end{array}$ $\begin{array}{lllll}-6.528 & 2.778 & -1.234 & 1.00 & 0.00\end{array}$ $\begin{array}{lllll}-5.802 & 3.114 & -1.961 & 1.00 & 0.00\end{array}$ $\begin{array}{lllll}-7.633 & 2.240 & -1.726 & 1.00 & 0.00\end{array}$ $\begin{array}{rrrrr}-8.464 & 1.848 & -0.759 & 1.00 & 0.00\end{array}$ $\begin{array}{lllll}-10.852 & -1.262 & -2.226 & 1.00 & 0.00 \\ -10.691 & -1.403 & -1.153 & 1.00 & 0.00\end{array}$ $\begin{array}{lllll}-10.691 & -1.403 & -1.153 & 1.00 & 0.00 \\ -9.800 & -0.315 & -2.785 & 1.00 & 0.00\end{array}$ $\begin{array}{lllll}-9.800 & -0.315 & -2.785 & 1.00 & 0.00 \\ -8.793 & -0.533 & -2.435 & 1.00 & 0.00\end{array}$ $\begin{array}{lllll}-8.793 & -0.533 & -2.435 & 1.00 & 0.00 \\ -9.810 & -0.220 & -4.178 & 1.00 & 0.00\end{array}$ $\begin{array}{rrrrr}-9.810 & -0.220 & -4.178 & 1.00 & 0.00 \\ -10.342 & -0.910 & -4.623 & 1.00 & 0.00\end{array}$ $\begin{array}{lllll}-10.342 & -0.910 & -4.623 & 1.00 & 0.00 \\ -10.913 & -2.501 & -2.890 & 1.00 & 0.00\end{array}$ $\begin{array}{lllll}-9.977 & -3.762 & -2.454 & 1.00 & 0.00\end{array}$ $\begin{array}{lllll}-10.372 & -4.851 & -3.403 & 1.00 & 0.00\end{array}$ $\begin{array}{lllll}-10.110 & -3.966 & -0.980 & 1.00 & 0.00\end{array}$ $\begin{array}{lllll}-8.446 & -3.262 & -2.724 & 1.00 & 0.00\end{array}$ $\begin{array}{lllll}-8.010 & -3.067 & -4.070 & 1.00 & 0.00\end{array}$ $\begin{array}{lllll}-8.761 & -2.522 & -4.643 & 1.00 & 0.00\end{array}$ $\begin{array}{lllll}-7.851 & -4.032 & -4.558 & 1.00 & 0.00\end{array}$ $\begin{array}{lllll}-6.703 & -2.316 & -4.200 & 1.00 & 0.00\end{array}$ $\begin{array}{lllll}-6.604 & -2.175 & -5.284 & 1.00 & 0.00\end{array}$ $\begin{array}{lllll}-6.691 & -1.047 & -3.551 & 1.00 & 0.00 \\ -5.378 & -0.685 & -3.203 & 1.00 & 0.00\end{array}$ $\begin{array}{rrrrr}-5.378 & -0.685 & -3.203 & 1.00 & 0.00 \\ -5.142 & 0.294 & -3.620 & 1.00 & 0.00\end{array}$ $\begin{array}{rrrrr}-5.142 & 0.294 & -3.620 & 1.00 & 0.00 \\ -5.253 & -0.586 & -1.736 & 1.00 & 0.00\end{array}$ $\begin{array}{lllll}-5.253 & -0.586 & -1.736 & 1.00 & 0.00 \\ -6.261 & -0.957 & -0.910 & 1.00 & 0.00\end{array}$ $\begin{array}{lllll}-7.145 & -1.370 & -1.370 & 1.00 & 0.00\end{array}$ $\begin{array}{lllll}-6.154 & -0.810 & 0.419 & 1.00 & 0.00\end{array}$ $\begin{array}{lllll}-6.959 & -1.079 & 1.075 & 1.00 & 0.00\end{array}$ $\begin{array}{lllll}-4.949 & -0.251 & 0.933 & 1.00 & 0.00\end{array}$ $\begin{array}{lllll}-4.804 & -0.124 & 2.239 & 1.00 & 0.00\end{array}$ $\begin{array}{lllll}-4.010 & 0.368 & 2.662 & 1.00 & 0.00\end{array}$ $\begin{array}{lllll}-5.551 & -0.432 & 2.844 & 1.00 & 0.00\end{array}$ $\begin{array}{lllll}-3.971 & 0.134 & 0.117 & 1.00 & 0.00\end{array}$ $-3.200-0.324$ $\begin{array}{llllll}-3.200 & 0.324 & -2.001 & 1.00 & 0.00 \\ -5.429 & -2.973 & -3.691 & 1.00 & 0.00\end{array}$ $\begin{array}{lllll}-5.429 & -2.973 & -3.691 & 1.00 & 0.00 \\ -5.556 & -3.256 & -2.641 & 1.00 & 0.00\end{array}$ $\begin{array}{lllll}-4.471 & -1.787 & -3.766 & 1.00 & 0.00\end{array}$ $\begin{array}{lllll}-3.574 & -1.922 & -3.169 & 1.00 & 0.00\end{array}$ $\begin{array}{lllll}-4.170 & -1.501 & -5.108 & 1.00 & 0.00\end{array}$ $\begin{array}{lllll}-3.210 & -1.500 & -5.277 & 1.00 & 0.00\end{array}$ $\begin{array}{lllll}-5.057 & -4.073 & -4.471 & 1.00 & 0.00\end{array}$ $\begin{array}{lllll}-4.225 & -5.286 & -3.774 & 1.00 & 0.00\end{array}$ $\begin{array}{lllll}-3.896 & -6.231 & -4.892 & 1.00 & 0.00\end{array}$ $\begin{array}{lllll}-4.929 & -5.746 & -2.532 & 1.00 & 0.00 \\ -2.876 & -4.565 & -3.240 & 1.00 & 0.00\end{array}$ $\begin{array}{llllll}-1.865 & -4.193 & -4.146 & 1.00 & 0.00\end{array}$ $\begin{array}{lllll}-2.259 & -3.538 & -4.929 & 1.00 & 0.00\end{array}$ $\begin{array}{lllll}-2.259 & -3.538 & -4.929 & 1.00 & 0.00\end{array}$ $\begin{array}{lllll}-0.760 & -3.472 & -3.420 & 1.00 & 0.00\end{array}$ $\begin{array}{lllll}-0.101 & -3.085 & -4.208 & 1.00 & 0.00\end{array}$ $\begin{array}{lllll}-1.284 & -2.399 & -2.642 & 1.00 & 0.00\end{array}$ $\begin{array}{lllll}-0.461 & -2.126 & -1.551 & 1.00 & 0.00\end{array}$ $\begin{array}{lllll}-0.101 & -1.097 & -1.601 & 1.00 & 0.00 \\ -1.214 & -2.239 & -0.292 & 1.00 & 0.00\end{array}$ $\begin{array}{lllll}-1.214 & -2.239 & -0.292 & 1.00 & 0.00 \\ -2.361 & -2.966 & -0.175 & 1.00 & 0.00\end{array}$ $\begin{array}{lllll}-2.361 & -2.966 & -0.175 & 1.00 & 0.00 \\ -2.706 & -3.460 & -1.072 & 1.00 & 0.00\end{array}$ $\begin{array}{lllll}-3.006 & -3.083 & 0.996 & 1.00 & 0.00\end{array}$ $\begin{array}{lllll}-3.889 & -3.696 & 1.069 & 1.00 & 0.00\end{array}$ $\begin{array}{lllll}-2.473 & -2.464 & 2.182 & 1.00 & 0.00\end{array}$ $\begin{array}{lllll}-2.945 & -2.554 & 3.303 & 1.00 & 0.00\end{array}$ $\begin{array}{lllll}-1.326 & -1.725 & 1.969 & 1.00 & 0.00\end{array}$ $\begin{array}{lllll}-0.874 & -1.271 & 2.767 & 1.00 & 0.00\end{array}$ $\begin{array}{rrrrr}-0.647 & -1.616 & 0.796 & 1.00 & 0.00 \\ 0.405 & -1.009 & 0.708 & 1.00 & 0.00\end{array}$ $\begin{array}{lllll}0.069 & -4.258 & -2.412 & 1.00 & 0.00\end{array}$ $\begin{array}{rrrrr}0.069 & -4.258 & -2.412 & 1.00 & 0.00 \\ -0.594 & -4.842 & -1.763 & 1.00 & 0.00\end{array}$ $\begin{array}{rrrrr}-0.594 & -4.842 & -1.763 & 1.00 & 0.00 \\ 0.710 & -3.122 & -1.620 & 1.00 & 0.00\end{array}$ $\begin{array}{lllll}0.710 & -3.122 & -1.620 & 1.00 & 0.00 \\ 1.054 & -3.444 & -0.634 & 1.00 & 0.00\end{array}$ $\begin{array}{lllll}1.054 & -3.444 & -0.634 & 1.00 & 0.00 \\ 1.751 & -2.560 & -2.376 & 1.00 & 0.00\end{array}$ $\begin{array}{lllll}2.235 & -1.931 & -1.813 & 1.00 & 0.00\end{array}$ $\begin{array}{lllll}0.978 & -5.089 & -3.076 & 1.00 & 0.00\end{array}$ $\begin{array}{lllll}1.677 & -6.355 & -2.351 & 1.00 & 0.00\end{array}$ $\begin{array}{lllll}1.984 & -7.357 & -3.420 & 1.00 & 0.00\end{array}$ $\begin{array}{lllll}0.884 & -6.783 & -1.148 & 1.00 & 0.00\end{array}$ $\begin{array}{lllll}3.054 & -5.640 & -1.861 & 1.00 & 0.00\end{array}$ $\begin{array}{lllll}4.080 & -6.400 & -1.238 & 1.00 & 0.00\end{array}$ $\begin{array}{lllll}4.211 & -7.371 & -1.731 & 1.00 & 0.00\end{array}$ $\begin{array}{lllll}3.843 & -6.600 & -0.189 & 1.00 & 0.00\end{array}$ $\begin{array}{lllll}5.390 & -5.641 & -1.311 & 1.00 & 0.00 \\ 6.137 & -6.247 & -0.795 & 1.00 & 0.00\end{array}$ 


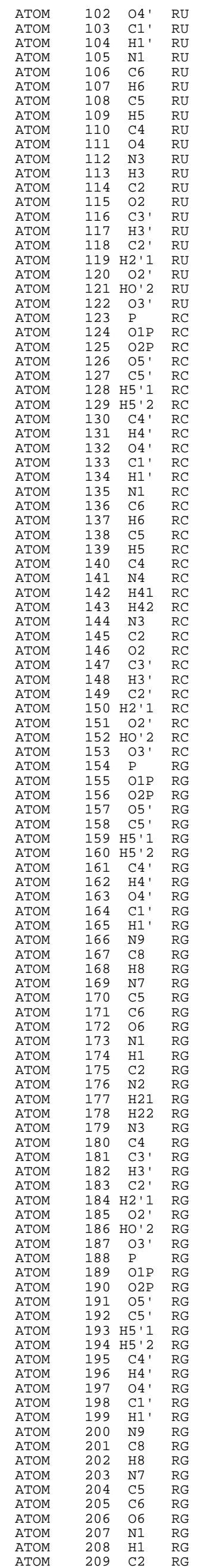

$\begin{array}{lllll}5.768 & -5.494 & -2.687 & 1.00 & 0.00\end{array}$

$\begin{array}{lllll}5.928 & -4.134 & -3.006 & 1.00 & 0.00\end{array}$

$\begin{array}{lllll}6.966 & -3.823 & -2.881 & 1.00 & 0.00 \\ 5.612 & -3.929 & -4.400 & 1.00 & 0.00\end{array}$

$\begin{array}{lllll}4.312 & -3.852 & -4.837 & 1.00 & 0.00\end{array}$

$\begin{array}{lllll}3.546 & -3.939 & -4.079 & 1.00 & 0.00\end{array}$

$\begin{array}{lllll}3.985 & -3.678 & -6.122 & 1.00 & 0.00\end{array}$

$\begin{array}{lllll}2.950 & -3.628 & -6.421 & 1.00 & 0.00\end{array}$

$\begin{array}{lllll}5.029 & -3.585 & -7.114 & 1.00 & 0.00\end{array}$

$\begin{array}{lllll}4.891 & -3.381 & -8.303 & 1.00 & 0.00\end{array}$

$\begin{array}{lllll}6.305 & -3.756 & -6.599 & 1.00 & 0.00\end{array}$

$\begin{array}{lllll}7.050 & -3.847 & -7.291 & 1.00 & 0.00\end{array}$

$\begin{array}{lllll}6.665 & -3.950 & -5.302 & 1.00 & 0.00\end{array}$

$\begin{array}{lllll}7.826 & -4.107 & -4.969 & 1.00 & 0.00\end{array}$

$\begin{array}{lllll}5.374 & -4.215 & -0.737 & 1.00 & 0.00\end{array}$

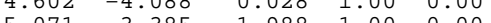

$\begin{array}{lllll}5.071 & -3.385 & -1.988 & 1.00 & 0.00 \\ 4.008 & -3.517 & -2.202 & 1.00 & 0.00\end{array}$

$\begin{array}{lllll}4.008 & -3.517 & -2.202 & 1.00 & 0.00 \\ 5.425 & -2.043 & -1.943 & 1.00 & 0.00\end{array}$

$\begin{array}{lllll}5.425 & -2.043 & -1.943 & 1.00 & 0.00 \\ 4.663 & -1.485 & -1.725 & 1.00 & 0.00\end{array}$

$\begin{array}{lllll}6.643 & -3.813 & -0.272 & 1.00 & 0.00\end{array}$

$\begin{array}{lllll}7.210 & -4.236 & 1.194 & 1.00 & 0.00\end{array}$

$\begin{array}{lllll}8.590 & -3.647 & 1.249 & 1.00 & 0.00\end{array}$

$\begin{array}{lllll}6.949 & -5.682 & 1.453 & 1.00 & 0.00\end{array}$

$\begin{array}{lllll}6.242 & -3.434 & 2.218 & 1.00 & 0.00\end{array}$

$\begin{array}{lllll}6.321 & -2.028 & 2.347 & 1.00 & 0.00\end{array}$

$\begin{array}{lllll}6.062 & -1.532 & 1.404 & 1.00 & 0.00\end{array}$

$\begin{array}{lllll}7.329 & -1.704 & 2.621 & 1.00 & 0.00\end{array}$

$\begin{array}{lllll}5.371 & -1.589 & 3.435 & 1.00 & 0.00 \\ 5.473 & -0.504 & 3.523 & 1.00 & 0.00\end{array}$

$\begin{array}{lllll}5.473 & -0.504 & 3.523 & 1.00 & 0.00 \\ 4.026 & -1.899 & 3.069 & 1.00 & 0.00\end{array}$

$\begin{array}{lllll}4.026 & -1.899 & 3.069 & 1.00 & 0.00 \\ 3.375 & -2.631 & 4.072 & 1.00 & 0.00\end{array}$

$\begin{array}{lllll}3.375 & -2.631 & 4.072 & 1.00 & 0.00 \\ 2.762 & -1.980 & 4.699 & 1.00 & 0.00\end{array}$

$\begin{array}{lllll}2.762 & -1.980 & 4.699 & 1.00 & 0.00 \\ 2.458 & -3.559 & 3.422 & 1.00 & 0.00\end{array}$

$\begin{array}{lllll}2.458 & -3.559 & 3.422 & 1.00 & 0.00 \\ 2.842 & -4.225 & 2.305 & 1.00 & 0.00\end{array}$

$\begin{array}{lllll}3.894 & -4.165 & 2.055 & 1.00 & 0.00\end{array}$

$\begin{array}{lllll}1.958 & -4.923 & 1.567 & 1.00 & 0.00\end{array}$

$\begin{array}{lllll}2.267 & -5.464 & 0.691 & 1.00 & 0.00\end{array}$

$\begin{array}{lllll}0.596 & -4.928 & 2.010 & 1.00 & 0.00\end{array}$

$\begin{array}{lllll}-0.353 & -5.485 & 1.265 & 1.00 & 0.00\end{array}$

$\begin{array}{lllll}-1.316 & -5.515 & 1.580 & 1.00 & 0.00\end{array}$

$\begin{array}{lllll}-0.097 & -5.987 & 0.423 & 1.00 & 0.00\end{array}$

$\begin{array}{lllll}0.257 & -4.372 & 3.161 & 1.00 & 0.00\end{array}$

$\begin{array}{lllll}1.160 & -3.712 & 3.907 & 1.00 & 0.00\end{array}$

$\begin{array}{lllll}0.872 & -3.240 & 5.003 & 1.00 & 0.00 \\ 5.613 & -2.241 & 4.797 & 1.00 & 0.00\end{array}$

$\begin{array}{lllll}5.613 & -2.241 & 4.797 & 1.00 & 0.00 \\ 6.603 & -2.696 & 4.877 & 1.00 & 0.00\end{array}$

$\begin{array}{lllll}6.603 & -2.696 & 4.877 & 1.00 & 0.00 \\ 4.504 & -3.290 & 4.885 & 1.00 & 0.00\end{array}$

$\begin{array}{lllll}4.504 & -3.290 & 4.885 & 1.00 & 0.00 \\ 4.832 & -4.194 & 4.370 & 1.00 & 0.00\end{array}$

$\begin{array}{lllll}4.832 & -4.194 & 4.370 & 1.00 & 0.00 \\ 4.166 & -3.654 & 6.184 & 1.00 & 0.00\end{array}$

$\begin{array}{lllll}4.055 & -2.843 & 6.693 & 1.00 & 0.00\end{array}$

$\begin{array}{lllll}5.391 & -1.301 & 5.838 & 1.00 & 0.00\end{array}$

$\begin{array}{lllll}6.648 & -0.617 & 6.614 & 1.00 & 0.00 \\ 7.645 & -1.681 & 6.958 & 1.00 & 0.00\end{array}$

$\begin{array}{lllll}7.645 & -1.681 & 6.958 & 1.00 & 0.00 \\ 6.029 & 0.224 & 7.696 & 1.00 & 0.00\end{array}$

$\begin{array}{lllll}6.029 & 0.224 & 7.696 & 1.00 & 0.00\end{array}$

$\begin{array}{lllll}7.326 & 0.333 & 5.486 & 1.00 & 0.00\end{array}$

$\begin{array}{lllll}6.636 & 1.520 & 5.133 & 1.00 & 0.00\end{array}$

$\begin{array}{lllll}6.480 & 2.146 & 6.014 & 1.00 & 0.00\end{array}$

$\begin{array}{lllll}5.657 & 1.295 & 4.702 & 1.00 & 0.00\end{array}$

$\begin{array}{lllll}7.425 & 2.317 & 4.122 & 1.00 & 0.00\end{array}$

$\begin{array}{lllll}8.405 & 2.598 & 4.523 & 1.00 & 0.00\end{array}$

$\begin{array}{lllll}7.594 & 1.575 & 2.924 & 1.00 & 0.00 \\ 7.408 & 2.373 & 1.788 & 1.00 & 0.00\end{array}$

$\begin{array}{lllll}7.408 & 2.373 & 1.788 & 1.00 & 0.00 \\ 8.344 & 2.509 & 1.240 & 1.00 & 0.00\end{array}$

$\begin{array}{rrrrr}8.344 & 2.509 & 1.240 & 1.00 & 0.00 \\ 6.528 & 1.702 & 0.874 & 1.00 & 0.00\end{array}$

$\begin{array}{rrrrr}6.528 & 1.702 & 0.874 & 1.00 & 0.00 \\ 6.794 & 1.453 & -0.448 & 1.00 & 0.00\end{array}$

$\begin{array}{lllll}7.740 & 1.738 & -0.874 & 1.00 & 0.00\end{array}$

$\begin{array}{lllll}5.804 & 0.903 & -1.072 & 1.00 & 0.00\end{array}$

$\begin{array}{lllll}4.836 & 0.762 & -0.108 & 1.00 & 0.00\end{array}$

$\begin{array}{lllll}3.586 & 0.124 & -0.148 & 1.00 & 0.00\end{array}$

$\begin{array}{lllll}3.117 & -0.488 & -1.128 & 1.00 & 0.00\end{array}$

$\begin{array}{lllll}2.894 & 0.224 & 1.035 & 1.00 & 0.00\end{array}$

$\begin{array}{lllll}1.967 & -0.209 & 1.048 & 1.00 & 0.00\end{array}$

$\begin{array}{lllll}3.357 & 0.844 & 2.161 & 1.00 & 0.00\end{array}$

$\begin{array}{lllll}2.536 & 0.945 & 3.199 & 1.00 & 0.00\end{array}$

$\begin{array}{lllll}2.906 & 1.358 & 4.047 & 1.00 & 0.00\end{array}$

$\begin{array}{lllll}1.660 & 0.439 & 3.280 & 1.00 & 0.00\end{array}$

$\begin{array}{lllll}1.564 & 1.374 & 2.236 & 1.00 & 0.00\end{array}$

$\begin{array}{lllll}5.254 & 1.283 & 1.103 & 1.00 & 0.00\end{array}$

$\begin{array}{lllll}5.254 & 1.283 & 1.103 & 1.00 & 0.00 \\ 6.636 & 3.569 & 3.735 & 1.00 & 0.00\end{array}$

$\begin{array}{lllll}5.573 & 3.379 & 3.887 & 1.00 & 0.00\end{array}$

$\begin{array}{lllll}6.878 & 3.747 & 2.232 & 1.00 & 0.00\end{array}$

$\begin{array}{lllll}5.936 & 3.995 & 1.726 & 1.00 & 0.00 \\ 7.863 & 4.702 & 1.926 & 1.00 & 0.00\end{array}$

$\begin{array}{lllll}7.663 & 5.513 & 2.420 & 1.00 & 0.00\end{array}$

$\begin{array}{lllll}7.053 & 4.736 & 4.387 & 1.00 & 0.00\end{array}$

$\begin{array}{lllll}6.539 & 5.291 & 5.823 & 1.00 & 0.00\end{array}$

$\begin{array}{lllll}6.768 & 6.771 & 5.725 & 1.00 & 0.00\end{array}$

$\begin{array}{lllll}7.134 & 4.467 & 6.926 & 1.00 & 0.00\end{array}$

$\begin{array}{lllll}4.949 & 4.975 & 5.827 & 1.00 & 0.00\end{array}$

$\begin{array}{lllll}3.990 & 5.857 & 5.259 & 1.00 & 0.00\end{array}$

$\begin{array}{lllll}4.271 & 6.904 & 5.398 & 1.00 & 0.00\end{array}$

$\begin{array}{lllll}3.056 & 5.680 & 5.796 & 1.00 & 0.00 \\ 3.793 & 5.582 & 3.783 & 1.00 & 0.00\end{array}$

$\begin{array}{lllll}3.793 & 5.582 & 3.783 & 1.00 & 0.00 \\ 4.747 & 5.729 & 3.260 & 1.00 & 0.00\end{array}$

$\begin{array}{lllll}4.747 & 5.729 & 3.260 & 1.00 & 0.00 \\ 3.363 & 4.235 & 3.615 & 1.00 & 0.00\end{array}$

$\begin{array}{lllll}3.363 & 4.235 & 3.615 & 1.00 & 0.00 \\ 2.446 & 4.118 & 2.557 & 1.00 & 0.00\end{array}$

$\begin{array}{lllll}2.795 & 3.388 & 1.825 & 1.00 & 0.00\end{array}$

$\begin{array}{lllll}1.179 & 3.651 & 3.060 & 1.00 & 0.00 \\ 0.716 & 3.669 & 4.348 & 1.00 & 0.00\end{array}$

$\begin{array}{lllll}1.272 & 4.122 & 5.154 & 1.00 & 0.00\end{array}$

$\begin{array}{lllll}-0.433 & 3.089 & 4.475 & 1.00 & 0.00\end{array}$

$\begin{array}{lllll}-0.757 & 2.666 & 3.211 & 1.00 & 0.00\end{array}$

$\begin{array}{lllll}-1.840 & 1.902 & 2.717 & 1.00 & 0.00\end{array}$

$\begin{array}{lllll}-2.778 & 1.417 & 3.349 & 1.00 & 0.00 \\ -1.758 & 1.687 & 1.352 & 1.00 & 0.00\end{array}$

$\begin{array}{lllll}-1.758 & 1.687 & 1.352 & 1.00 & 0.00\end{array}$

$\begin{array}{lllll}-2.537 & 1.155 & 0.924 & 1.00 & 0.00 \\ -0.724 & 2.069 & 0.550 & 1.00 & 0.00\end{array}$ 


$\begin{array}{llll}\text { ATOM } & 210 & \text { N2 } & \text { RG } \\ \text { ATOM } & 211 & \text { H21 } & \text { RG } \\ \text { ATOM } & 212 & \text { H22 } & \text { RG } \\ \text { ATOM } & 213 & \text { N3 } & \text { RG } \\ \text { ATOM } & 214 & \text { C4 } & \text { RG } \\ \text { ATOM } & 215 & \text { C3' } & \text { RG } \\ \text { ATOM } & 216 & \text { H3' } & \text { RG } \\ \text { ATOM } & 217 & \text { C2' } & \text { RG } \\ \text { ATOM } & 218 & \text { H2'' } & \text { RG } \\ \text { ATOM } & 219 & \text { O2' } & \text { RG } \\ \text { ATOM } & 220 & \text { HO'2 } & \text { RG } \\ \text { ATOM } & 221 & \text { O3' } & \text { RG } \\ \text { ATOM } & 222 & \text { P } & \text { RU3 } \\ \text { ATOM } & 223 & \text { O1P } & \text { RU3 } \\ \text { ATOM } & 224 & \text { O2P } & \text { RU3 } \\ \text { ATOM } & 225 & \text { O5' } & \text { RU3 } \\ \text { ATOM } & 226 & \text { C5' } & \text { RU3 } \\ \text { ATOM } & 227 & \text { H5'1 } & \text { RU3 } \\ \text { ATOM } & 228 & \text { H5' } & \text { RU3 } \\ \text { ATOM } & 229 & \text { C4' } & \text { RU3 } \\ \text { ATOM } & 230 & \text { H4' } & \text { RU3 } \\ \text { ATOM } & 231 & \text { O4' } & \text { RU3 } \\ \text { ATOM } & 232 & \text { C1' } & \text { RU3 } \\ \text { ATOM } & 233 & \text { H1' } & \text { RU3 } \\ \text { ATOM } & 234 & \text { N1 } & \text { RU3 } \\ \text { ATOM } & 235 & \text { C6 } & \text { RU3 } \\ \text { ATOM } & 236 & \text { H6 } & \text { RU3 } \\ \text { ATOM } & 237 & \text { C5 } & \text { RU3 } \\ \text { ATOM } & 238 & \text { H5 } & \text { RU3 } \\ \text { ATOM } & 239 & \text { C4 } & \text { RU3 } \\ \text { ATOM } & 240 & \text { O4 } & \text { RU3 } \\ \text { ATOM } & 241 & \text { N3 } & \text { RU3 } \\ \text { ATOM } & 242 & \text { H3 } & \text { RU3 } \\ \text { ATOM } & 243 & \text { C2 } & \text { RU3 } \\ \text { ATOM } & 244 & \text { O2 } & \text { RU3 } \\ \text { ATOM } & 245 & \text { C3' } & \text { RU3 } \\ \text { ATOM } & 246 & \text { H3' } & \text { RU3 } \\ \text { ATOM } & 247 & \text { C2' } & \text { RU3 } \\ \text { ATOM } & 248 & \text { H2'1 } & \text { RU3 } \\ \text { ATOM } & 249 & \text { O2' } & \text { RU3 } \\ \text { ATOM } & 250 & \text { HO'2 } & \text { RU3 } \\ \text { ATOM } & 251 & \text { O3' } & \text { RU3 } \\ \text { ATOM } & 252 & \text { H3T } & \text { RU3 } \\ \text { END } & & & \\ & & & \end{array}$

$\begin{array}{rrrrr}-0.831 & 1.742 & -0.739 & 1.00 & 0.00 \\ 0.004 & 1.812 & -1.307 & 1.00 & 0.00 \\ -1.628 & 1.227 & -1.095 & 1.00 & 0.00 \\ 0.319 & 2.729 & 1.004 & 1.00 & 0.00 \\ 0.243 & 2.999 & 2.314 & 1.00 & 0.00 \\ 2.700 & 6.398 & 3.102 & 1.00 & 0.00 \\ 1.833 & 6.468 & 3.772 & 1.00 & 0.00 \\ 2.338 & 5.500 & 1.925 & 1.00 & 0.00 \\ 1.335 & 5.690 & 1.540 & 1.00 & 0.00 \\ 3.316 & 5.622 & 0.924 & 1.00 & 0.00 \\ 2.991 & 5.148 & 0.126 & 1.00 & 0.00 \\ 3.158 & 7.668 & 2.730 & 1.00 & 0.00 \\ 2.046 & 8.860 & 2.556 & 1.00 & 0.00 \\ 2.846 & 10.068 & 2.154 & 1.00 & 0.00 \\ 1.180 & 8.888 & 3.770 & 1.00 & 0.00 \\ 1.144 & 8.345 & 1.321 & 1.00 & 0.00 \\ 1.679 & 8.317 & 0.005 & 1.00 & 0.00 \\ 2.581 & 7.706 & -0.046 & 1.00 & 0.00 \\ 1.932 & 9.323 & -0.339 & 1.00 & 0.00 \\ 0.626 & 7.790 & -0.940 & 1.00 & 0.00 \\ 1.088 & 7.713 & -1.934 & 1.00 & 0.00 \\ 0.127 & 6.522 & -0.536 & 1.00 & 0.00 \\ -1.221 & 6.381 & -0.911 & 1.00 & 0.00 \\ -1.315 & 5.672 & -1.731 & 1.00 & 0.00 \\ -1.975 & 5.826 & 0.207 & 1.00 & 0.00 \\ -1.970 & 6.451 & 1.427 & 1.00 & 0.00 \\ -1.211 & 7.212 & 1.564 & 1.00 & 0.00 \\ -2.832 & 6.134 & 2.402 & 1.00 & 0.00 \\ -2.811 & 6.659 & 3.343 & 1.00 & 0.00 \\ -3.837 & 5.119 & 2.172 & 1.00 & 0.00 \\ -4.738 & 4.824 & 2.940 & 1.00 & 0.00 \\ -3.724 & 4.483 & 0.953 & 1.00 & 0.00 \\ -4.471 & 3.827 & 0.672 & 1.00 & 0.00 \\ -2.857 & 4.796 & -0.049 & 1.00 & 0.00 \\ -2.889 & 4.201 & -1.107 & 1.00 & 0.00 \\ -0.586 & 8.708 & -1.015 & 1.00 & 0.00 \\ -0.765 & 9.154 & -0.031 & 1.00 & 0.00 \\ -1.751 & 7.764 & -1.355 & 1.00 & 0.00 \\ -2.651 & 8.030 & -0.786 & 1.00 & 0.00 \\ -2.026 & 7.728 & -2.722 & 1.00 & 0.00 \\ -2.238 & 8.639 & -3.004 & 1.00 & 0.00 \\ -0.344 & 9.696 & -1.971 & 1.00 & 0.00 \\ -0.777 & 10.524 & -1.671 & 1.00 & 0.00 \\ & & & & \end{array}$

\section{D-180 deg-QM.pdb}

\begin{tabular}{|c|c|c|c|}
\hline ATOM & 1 & H5T & RA 5 \\
\hline ATOM & 2 & $05^{\prime}$ & RA5 \\
\hline ATOM & 3 & C5' & RA5 \\
\hline ATOM & 4 & H5' 1 & RA5 \\
\hline ATOM & 5 & H5' 2 & RA5 \\
\hline ATOM & 6 & $\mathrm{C} 44^{\prime}$ & RA5 \\
\hline ATOM & 7 & H4 ${ }^{\prime}$ & RA5 \\
\hline ATOM & 8 & O4' & RA5 \\
\hline ATOM & 9 & $\mathrm{C} 1^{\prime}$ & RA5 \\
\hline ATOM & 10 & $\mathrm{H} 1^{\prime}$ & RA5 \\
\hline ATOM & 11 & N9 & RA5 \\
\hline ATOM & 12 & C8 & RA5 \\
\hline АTOM & 13 & H8 & RA5 \\
\hline ATOM & 14 & N7 & RA5 \\
\hline ATOM & 15 & C5 & RA5 \\
\hline ATOM & 16 & C6 & RA5 \\
\hline ATOM & 17 & N6 & RA5 \\
\hline ATOM & 18 & H61 & RA5 \\
\hline ATOM & 19 & H 62 & RA5 \\
\hline ATOM & 20 & N1 & RA5 \\
\hline ATOM & 21 & C2 & RA5 \\
\hline ATOM & 22 & H2 & RA5 \\
\hline ATOM & 23 & N3 & RA5 \\
\hline ATOM & 24 & $\mathrm{C} 4$ & RA5 \\
\hline ATOM & 25 & C3' & RA5 \\
\hline ATOM & 26 & H3 ${ }^{\prime}$ & RA5 \\
\hline ATOM & 27 & C2 ${ }^{\prime}$ & RA5 \\
\hline ATOM & 28 & H2' 1 & RA5 \\
\hline ATOM & 29 & $02^{\prime}$ & RA5 \\
\hline ATOM & 30 & HO' 2 & RA5 \\
\hline ATOM & 31 & O3' & RA5 \\
\hline ATOM & 32 & $\mathrm{P}$ & $\mathrm{RC}$ \\
\hline ATOM & 33 & $01 \mathrm{P}$ & $\mathrm{RC}$ \\
\hline ATOM & 34 & $\mathrm{O} 2 \mathrm{P}$ & $\mathrm{RC}$ \\
\hline ATOM & 35 & $05^{\prime}$ & $\mathrm{RC}$ \\
\hline ATOM & 36 & C5 ${ }^{\prime}$ & $\mathrm{RC}$ \\
\hline ATOM & 37 & H5'1 & $\mathrm{RC}$ \\
\hline ATOM & 38 & H5' 2 & $\mathrm{RC}$ \\
\hline ATOM & 39 & C4 ${ }^{\prime}$ & $\mathrm{RC}$ \\
\hline ATOM & 40 & H4 ' & $\mathrm{RC}$ \\
\hline ATOM & 41 & O4' & $\mathrm{RC}$ \\
\hline ATOM & 42 & $\mathrm{C} 1^{\prime}$ & $\mathrm{RC}$ \\
\hline ATOM & 43 & $\mathrm{H} 1^{\prime}$ & $\mathrm{RC}$ \\
\hline ATOM & 44 & N1 & $\mathrm{RC}$ \\
\hline ATOM & 45 & C6 & $\mathrm{RC}$ \\
\hline ATOM & 46 & H6 & $\mathrm{RC}$ \\
\hline ATOM & 47 & C5 & $\mathrm{RC}$ \\
\hline ATOM & 48 & H5 & $\mathrm{RC}$ \\
\hline ATOM & 49 & C4 & $\mathrm{RC}$ \\
\hline ATOM & 50 & N4 & $\mathrm{RC}$ \\
\hline ATOM & 51 & H 41 & $\mathrm{RC}$ \\
\hline ATOM & 52 & $\mathrm{H} 42$ & $\mathrm{RC}$ \\
\hline ATOM & 53 & N3 & $\mathrm{RC}$ \\
\hline ATOM & 54 & C2 & $\mathrm{RC}$ \\
\hline ATOM & 55 & 02 & $\mathrm{RC}$ \\
\hline ATOM & 56 & C3' & $\mathrm{RC}$ \\
\hline ATOM & 57 & H3' & $\mathrm{RC}$ \\
\hline ATOM & 58 & C2 ${ }^{\prime}$ & $\mathrm{RC}$ \\
\hline ATOM & 59 & H2' 1 & $\mathrm{RC}$ \\
\hline ATOM & 60 & $\mathrm{O} 2^{\prime}$ & $\mathrm{RC}$ \\
\hline ATOM & 61 & HO' 2 & $\mathrm{RC}$ \\
\hline & & & \\
\hline
\end{tabular}

\begin{tabular}{|c|c|c|c|c|}
\hline-13.315 & 0.211 & -0.040 & 1.00 & 0.00 \\
\hline-13.194 & -0.736 & -0.286 & 1.00 & 0.00 \\
\hline$-13 \cdot 346$ & -0.845 & -1.679 & 1.00 & 0.00 \\
\hline-14.208 & -0.275 & -2.036 & 1.00 & 0.00 \\
\hline-13.562 & -1.890 & -1.926 & 1.00 & 0.00 \\
\hline$-12 \cdot 120$ & -0.443 & -2.465 & 1.00 & 0.00 \\
\hline-12.400 & -0.501 & -3.526 & 1.00 & 0.00 \\
\hline-11.705 & 0.890 & -2.140 & 1.00 & 0.00 \\
\hline$-10 \cdot 311$ & 1.013 & -2.218 & 1.00 & 0.00 \\
\hline-10.043 & 1.863 & -2.849 & 1.00 & 0.00 \\
\hline-9.700 & 1.269 & -0.910 & 1.00 & 0.00 \\
\hline-10.155 & 1.032 & 0.349 & 1.00 & 0.00 \\
\hline-11.113 & 0.576 & 0.530 & 1.00 & 0.00 \\
\hline-9.324 & 1.425 & 1.280 & 1.00 & 0.00 \\
\hline-8.255 & 1.946 & 0.594 & 1.00 & 0.00 \\
\hline-7.056 & 2.565 & 1.007 & 1.00 & 0.00 \\
\hline-6.727 & 2.782 & 2.275 & 1.00 & 0.00 \\
\hline-5.937 & 3.371 & 2.521 & 1.00 & 0.00 \\
\hline-7.322 & 2.471 & 3.026 & 1.00 & 0.00 \\
\hline-6.209 & 2.961 & 0.044 & 1.00 & 0.00 \\
\hline-6.532 & 2.781 & -1.234 & 1.00 & 0.00 \\
\hline-5.797 & 3.119 & -1.955 & 1.00 & 0.00 \\
\hline-7.631 & 2.238 & -1.728 & 1.00 & 0.00 \\
\hline-8.467 & 1.849 & -0.768 & 1.00 & 0.00 \\
\hline-10.851 & -1.254 & -2.240 & 1.00 & 0.00 \\
\hline-10.696 & -1.397 & -1.164 & 1.00 & 0.00 \\
\hline-9.799 & -0.304 & -2.799 & 1.00 & 0.00 \\
\hline-8.793 & -0.529 & -2.446 & 1.00 & 0.00 \\
\hline-9.815 & -0.209 & -4.193 & 1.00 & 0.00 \\
\hline-10.333 & -0.912 & -4.634 & 1.00 & 0.00 \\
\hline-10.911 & -2.493 & -2.902 & 1.00 & 0.00 \\
\hline-9.966 & -3.751 & -2.468 & 1.00 & 0.00 \\
\hline-10.352 & -4.844 & -3.419 & 1.00 & 0.00 \\
\hline-10.099 & -3.958 & -0.992 & 1.00 & 0.00 \\
\hline-8.437 & -3.232 & -2.734 & 1.00 & 0.00 \\
\hline-8.004 & -3.031 & -4.084 & 1.00 & 0.00 \\
\hline-8.760 & -2.490 & -4.654 & 1.00 & 0.00 \\
\hline-7.851 & -3.998 & -4.572 & 1.00 & 0.00 \\
\hline-6.694 & -2.288 & -4.214 & 1.00 & 0.00 \\
\hline-6.598 & -2.146 & -5.297 & 1.00 & 0.00 \\
\hline-6.669 & -1.024 & -3.563 & 1.00 & 0.00 \\
\hline-5.357 & -0.678 & -3.203 & 1.00 & 0.00 \\
\hline-5.100 & 0.297 & -3.616 & 1.00 & 0.00 \\
\hline-5.238 & -0.581 & -1.734 & 1.00 & 0.00 \\
\hline-6.252 & -0.941 & -0.914 & 1.00 & 0.00 \\
\hline-7.133 & -1.346 & -1.383 & 1.00 & 0.00 \\
\hline-6.147 & -0.795 & 0.417 & 1.00 & 0.00 \\
\hline-6.961 & -1.060 & 1.065 & 1.00 & 0.00 \\
\hline-4.937 & -0.248 & 0.934 & 1.00 & 0.00 \\
\hline-4.800 & -0.122 & 2.240 & 1.00 & 0.00 \\
\hline-4.007 & 0.359 & 2.667 & 1.00 & 0.00 \\
\hline-5.550 & -0.414 & 2.847 & 1.00 & 0.00 \\
\hline & 0.123 & 0.124 & 1.00 & 0.00 \\
\hline-4.065 & -0.029 & -1.206 & 1.00 & 0.00 \\
\hline-3.170 & 0.288 & -1.993 & 1.00 & 0.00 \\
\hline-5.430 & -2.961 & -3.706 & 1.00 & 0.00 \\
\hline & -3.241 & -2.654 & 1.00 & 0.00 \\
\hline & -1.783 & -3.764 & 1.00 & 0.00 \\
\hline-3.568 & -1.940 & -3.156 & 1.00 & 0.00 \\
\hline & -1.495 & -5.099 & 1.00 & 0.00 \\
\hline-3.174 & -1.461 & -5.249 & 1.00 & 0.00 \\
\hline-5.081 & -4.064 & -4.486 & 1.00 & 0.00 \\
\hline
\end{tabular}




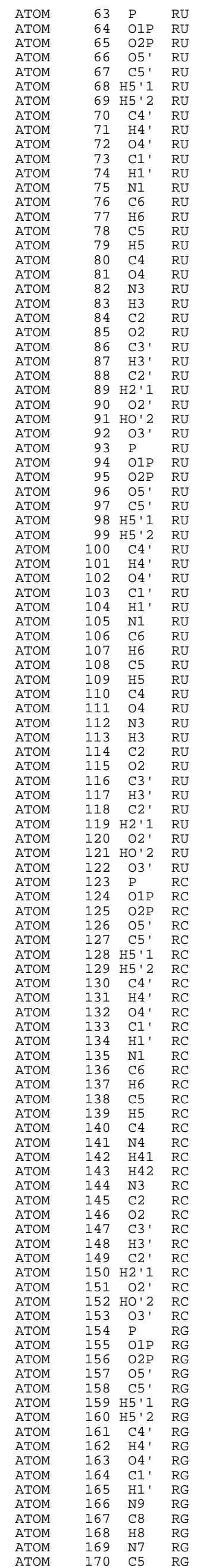

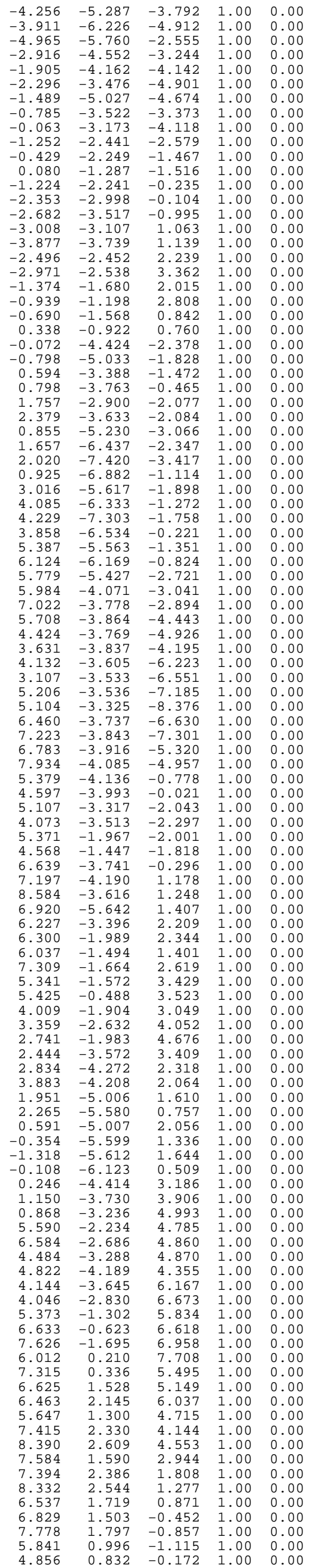




\begin{tabular}{|c|c|c|c|}
\hline ATOM & 171 & $\mathrm{C} 6$ & RG \\
\hline ATOM & 172 & 06 & RG \\
\hline ATOM & 173 & N1 & RG \\
\hline ATOM & 174 & H1 & RG \\
\hline ATOM & 175 & C2 & RG \\
\hline ATOM & 176 & $\mathrm{~N} 2$ & RG \\
\hline ATOM & 177 & H 21 & RG \\
\hline ATOM & 178 & $\mathrm{H} 22$ & RG \\
\hline ATOM & 179 & N3 & RG \\
\hline ATOM & 180 & C4 & RG \\
\hline ATOM & 181 & C3' & RG \\
\hline ATOM & 182 & H3' & RG \\
\hline ATOM & 183 & $\mathrm{C} 2{ }^{\prime}$ & RG \\
\hline ATOM & 184 & H2' 1 & RG \\
\hline ATOM & 185 & $02^{\prime}$ & RG \\
\hline ATOM & 186 & HO' 2 & RG \\
\hline ATOM & 187 & $03^{\prime}$ & RG \\
\hline ATOM & 188 & P & RG \\
\hline ATOM & 189 & $01 \mathrm{P}$ & RG \\
\hline ATOM & 190 & $\mathrm{O} 2 \mathrm{P}$ & RG \\
\hline ATOM & 191 & $05^{\prime}$ & RG \\
\hline ATOM & 192 & C5' & RG \\
\hline ATOM & 193 & H5' 1 & RG \\
\hline ATOM & 194 & H5' 2 & RG \\
\hline ATOM & 195 & C4 ${ }^{\prime}$ & RG \\
\hline ATOM & 196 & H4 ${ }^{\prime}$ & RG \\
\hline ATOM & 197 & $04^{\prime}$ & RG \\
\hline ATOM & 198 & $\mathrm{C} 1^{\prime}$ & RG \\
\hline ATOM & 199 & H1' & RG \\
\hline ATOM & 200 & N9 & RG \\
\hline ATOM & 201 & C 8 & RG \\
\hline ATOM & 202 & H8 & RG \\
\hline ATOM & 203 & N7 & RG \\
\hline ATOM & 204 & C5 & RG \\
\hline ATOM & 205 & C6 & RG \\
\hline ATOM & 206 & 06 & RG \\
\hline ATOM & 207 & N1 & RG \\
\hline ATOM & 208 & H1 & RG \\
\hline ATOM & 209 & $\mathrm{C} 2$ & RG \\
\hline ATOM & 210 & N2 & RG \\
\hline ATOM & 211 & $\mathrm{H} 21$ & RG \\
\hline ATOM & 212 & $\mathrm{H} 22$ & RG \\
\hline ATOM & 213 & N3 & RG \\
\hline ATOM & 214 & $\mathrm{C} 4$ & RG \\
\hline ATOM & 215 & C3' & RG \\
\hline ATOM & 216 & H3 ${ }^{\prime}$ & RG \\
\hline ATOM & 217 & C2 ${ }^{\prime}$ & RG \\
\hline ATOM & 218 & H2' 1 & RG \\
\hline ATOM & 219 & $02^{\prime}$ & RG \\
\hline ATOM & 220 & $\mathrm{HO}^{\prime} 2$ & RG \\
\hline ATOM & 221 & $03^{\prime}$ & RG \\
\hline ATOM & 222 & $\mathrm{P}$ & RU3 \\
\hline ATOM & 223 & $\mathrm{O} 1 \mathrm{P}$ & RU3 \\
\hline ATOM & 224 & $\mathrm{O} 2 \mathrm{P}$ & RU3 \\
\hline ATOM & 225 & $05^{\prime}$ & RU3 \\
\hline ATOM & 226 & C5' & RU3 \\
\hline ATOM & 227 & H5' 1 & RU3 \\
\hline ATOM & 228 & H5' 2 & RU3 \\
\hline ATOM & 229 & C4' & RU3 \\
\hline ATOM & 230 & H4 & RU3 \\
\hline ATOM & 231 & $04^{\prime}$ & RU3 \\
\hline ATOM & 232 & $\mathrm{C} 1^{\prime}$ & RU3 \\
\hline ATOM & 233 & H1' & RU3 \\
\hline ATOM & 234 & N1 & RU3 \\
\hline ATOM & 235 & C6 & RU3 \\
\hline ATOM & 236 & H6 & RU3 \\
\hline ATOM & 237 & C5 & RU3 \\
\hline ATOM & 238 & H5 & RU3 \\
\hline ATOM & 239 & $\mathrm{C} 4$ & RU3 \\
\hline ATOM & 240 & 04 & RU3 \\
\hline ATOM & 241 & N3 & RU3 \\
\hline ATOM & 242 & H3 & RU3 \\
\hline ATOM & 243 & $\mathrm{C} 2$ & RU3 \\
\hline ATOM & 244 & 02 & RU3 \\
\hline ATOM & 245 & C3' & RU3 \\
\hline ATOM & 246 & H3 ${ }^{\prime}$ & RU3 \\
\hline ATOM & 247 & C2' & RU3 \\
\hline ATOM & 248 & $\mathrm{H} 2{ }^{\prime} 1$ & RU3 \\
\hline ATOM & 249 & $\mathrm{O} 22^{\prime}$ & RU3 \\
\hline ATOM & 250 & HO' 2 & RU3 \\
\hline ATOM & 251 & $03^{\prime}$ & RU3 \\
\hline ATOM & 252 & Н3 T & RU3 \\
\hline
\end{tabular}

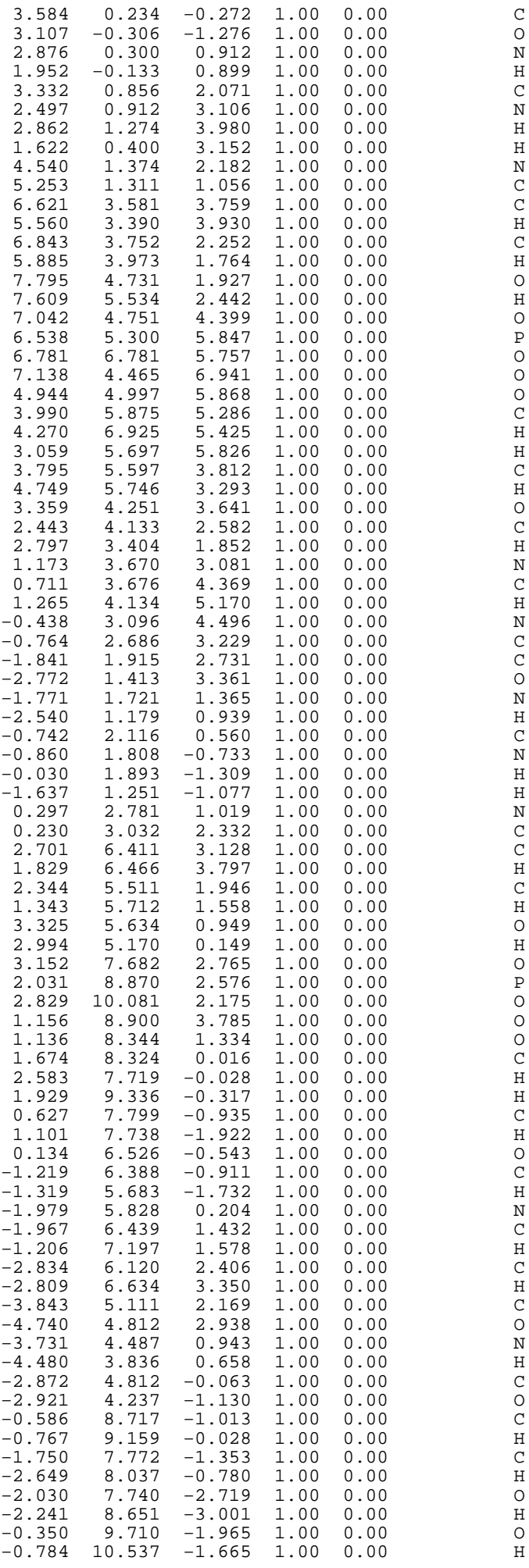

